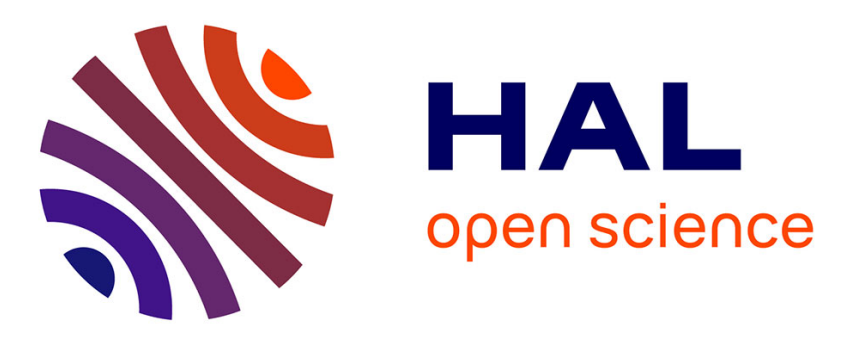

\title{
Structural, electronic and magnetic properties of some early vs late transition dimetallaborane clusters - A theoretical investigation
}

Kandasamy Bharathi, Lalshab Beerma, Chinnasamy Santhi, Bellie Sundaram Krishnamoorthy, Jean-François Halet

\section{To cite this version:}

Kandasamy Bharathi, Lalshab Beerma, Chinnasamy Santhi, Bellie Sundaram Krishnamoorthy, JeanFrançois Halet. Structural, electronic and magnetic properties of some early vs late transition dimetallaborane clusters - A theoretical investigation. Journal of Organometallic Chemistry, 2015, 792, pp.220-228. 10.1016/j.jorganchem.2015.05.057 . hal-01158448

HAL Id: hal-01158448

https://hal-univ-rennes1.archives-ouvertes.fr/hal-01158448

Submitted on 12 Nov 2015

HAL is a multi-disciplinary open access archive for the deposit and dissemination of scientific research documents, whether they are published or not. The documents may come from teaching and research institutions in France or abroad, or from public or private research centers.
L'archive ouverte pluridisciplinaire HAL, est destinée au dépôt et à la diffusion de documents scientifiques de niveau recherche, publiés ou non, émanant des établissements d'enseignement et de recherche français ou étrangers, des laboratoires publics ou privés. 
For the table of contents use only

\section{Structural, electronic and magnetic properties of some early vs late transition dimetallaborane clusters - A theoretical investigation}

Kandasamy Bharathi ${ }^{\mathrm{a}}$, Lalshab Beerma ${ }^{\mathrm{a}}$, Chinnasamy Santhi ${ }^{\mathrm{a}}$, Bellie Sundaram Krishnamoorthy ${ }^{\mathrm{a}, \mathrm{b}, *}$ and Jean-François Halet ${ }^{\mathrm{b}, *}$

${ }^{a}$ Department of Chemistry, Vivekanandha College of Arts and Sciences for Women (Autonomous), Elayampalayam, Tiruchengode, 600 036, India

${ }^{\mathrm{b}}$ Institut des Sciences Chimiques de Rennes, UMR 6226 CNRS-Université de Rennes 1, Avenue du Général Leclerc, 35042 Rennes Cédex, France

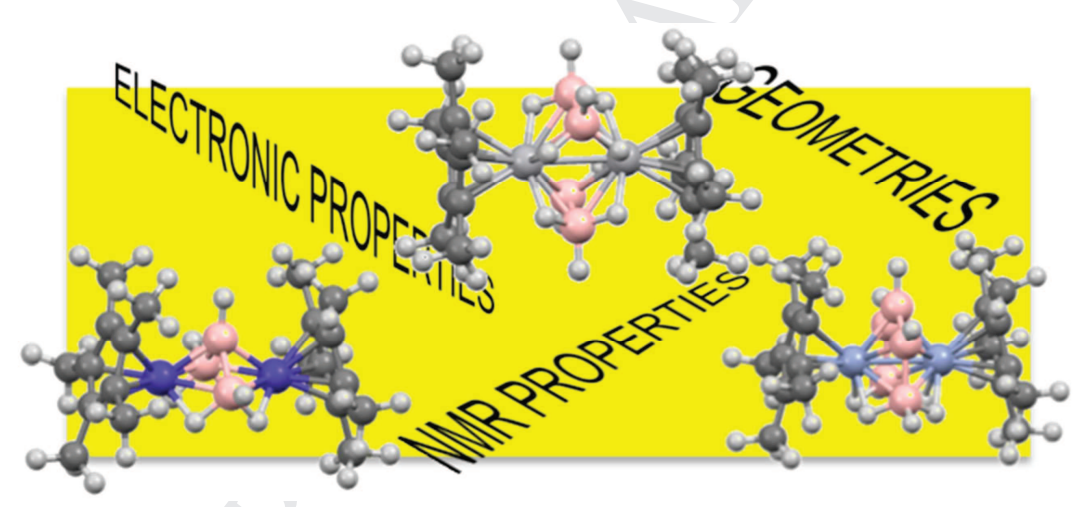




\section{Structural, electronic and magnetic properties of some early vs late transition dimetallaborane clusters - A theoretical investigation}

Kandasamy Bharathi ${ }^{\mathrm{a}}$, Lalshab Beerma ${ }^{\mathrm{a}}$, Chinnasamy Santhi ${ }^{\mathrm{a}}$, Bellie Sundaram Krishnamoorthy $^{\mathrm{a}, \mathrm{b}, *}$ and Jean-François Halet ${ }^{\mathrm{b}, *}$

${ }^{a}$ Department of Chemistry, Vivekanandha College of Arts and Sciences for Women (Autonomous), Elayampalayam, Tiruchengode, 600 036, India

${ }^{\mathrm{b}}$ Institut des Sciences Chimiques de Rennes, UMR 6226 CNRS-Université de Rennes 1, Avenue du Général Leclerc, 35042 Rennes Cédex, France

ABSTRACT. The strength of DFT methods in analyzing the electronic and magnetic properties of a series of dimetallaboranes of varied stoichiometry and architectural core, namely $\mathrm{M}_{2} \mathrm{~B}_{3}, \mathrm{M}_{2} \mathrm{~B}_{4}$ and $\mathrm{M}_{2} \mathrm{~B}_{5}$ with both early- and late-transition metals is demonstrated. In particular, the observed ${ }^{1} \mathrm{H}$ and ${ }^{11} \mathrm{~B}$ chemical shifts of most of the studied compounds are reproduced with a good accuracy of a few ppm at the DFT-GIAO BP86/TZ2P/SC level for the compounds with first-row transition metal elements and at the B3LYP/TZ2P/SO level for those with second- and third-row transition metal elements. This allows structural applications in elucidating the number and the location of bridging hydrogen atoms in experimentally poorly characterized metallaboranes such as $(\mathrm{Cp} * \mathrm{Cr})_{2} \mathrm{~B}_{4} \mathrm{H}_{8}$.

Dedicated to Prof. Michael Mingos on the occasion of his $70^{\text {th }}$ birthday in recognition of his major contributions to cluster chemistry.

Keywords: Cluster compounds . Density functional theory . Metallaborane complexes. NMR spectroscopy

* Corresponding authors. E-mail addresses: bskimo@yahoo.co.in (B. S. Krishnamoorthy) and halet@univ-rennes1.fr(J.-F. Halet) 


\section{Introduction}

Although the proven methods for the formation of compounds containing metal-boron bonds are rather limited, there are today a plethora of metallaborane compounds, which have been synthesized and characterized with nearly all the transition metals, early and late metals [1]. Among them, dimetallaboranes constitute a larger part, with almost one hundred of them known and structurally and/or spectroscopically characterized [2-22]. Although the chemistry of these dimetallaboranes is experimentally growing rapidly, theoretical studies on these species are still rather scarce, despite the need to investigate and rationalize viz., (i) their structural diversity, (ii) their thermal and kinetic stability, (iii) their isomeric preferences, (iv) their chemical bonding, (v) their spectroscopic properties, etc. In particular, quantum chemical computations of ${ }^{11} \mathrm{~B}$ and ${ }^{1} \mathrm{H}$ NMR chemical shifts have become one of the principal means of characterization of metallaborane compounds. These computations can even sometimes "rival that of X-ray crystallography" [24]. Density-functional theory (DFT) computations for instance can nowadays provide usefully precise chemical shifts as a function of the geometrical structure [25]. This has been shown by the past for some metallacarboranes for instance [26,27]. Recently, we have used such methods to tackle with some success the structural, electronic, and NMR properties of specific examples of dimetallaboranes [29-32].

For the latter, accuracies of about 2 to $3 \mathrm{ppm}$ were achieved for the computed ${ }^{11} \mathrm{~B}$ chemical shifts at the B3LYP/TZ2P all-electron relativistic scalar ZORA level of theory. Indeed, we have shown that although they are very computationally demanding, ${ }^{11} \mathrm{~B}$ NMR chemical shift calculations at this level of theory using BP86/TZ2P/SC optimized geometries improves 
considerably (by ca. 5-10 ppm) the computed values with respect to those obtained at the GGA/TZ2P all-electron relativistic scalar ZORA level, for early $4 d$ transition metal-borane systems (dimolybdaboranes, ditantalaboranes) [29,30]. On the other hand, only 1-2 ppm of improvement is observed for the metallaboranes with late $4 d$ transition metal (diruthenaboranes for instance) [31]. This drove us to look at the suitability of DFT methods using different GGA vs. hybrid functionals to accurately compute ${ }^{11} \mathrm{~B}$ NMR chemical shifts in metallaboranes in general. For this purpose a series of dimetallaboranes of varied stoichiometry and architectural cores, namely $\mathrm{M}_{2} \mathrm{~B}_{3}, \mathrm{M}_{2} \mathrm{~B}_{4}$ and $\mathrm{M}_{2} \mathrm{~B}_{5}$, with early and late transition metals has been chosen and studied (Chart 1). It would have been desirable to compare results on early- and late-transitionmetal boranes with the same architecture, but it has been shown experimentally that early transition metals often lead to the formation of rather highly condensed metallaborane clusters (of $\mathrm{M}_{2} \mathrm{~B}_{4}$ and $\mathrm{M}_{2} \mathrm{~B}_{5}$ core), such as compounds 1-9 (Chart 1) discussed in this work, for example $[13,33,34]$ whereas late-transition elements usually form stable metallaboranes with more open structures (with $\mathrm{M}_{2} \mathrm{~B}_{3}$ core), such as compounds 15-17 (Chart 1) discussed in this work, for example [35]. Here we report that the observed ${ }^{11} \mathrm{~B}$ NMR chemical shifts are reproduced with a reasonable accuracy at the $\mathrm{BP} 86 / \mathrm{TZ2} / \mathrm{SC}$ level for the $3 d$ metallaboranes. On the other hand, the $\mathrm{B} 3 \mathrm{LYP} / \mathrm{TZ2} / \mathrm{SO}$ level ( $\mathrm{SO}=$ spin-orbit) is necessary for $4 d$ and $5 d$ metallaboranes using BP86-optimized molecular geometries. This suggests that this particular combination of DFT levels is suitable for metallaboranes in general. 


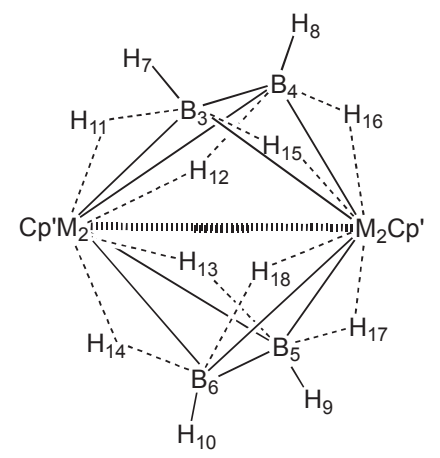

$1 \mathrm{M}=\mathrm{V}, \mathrm{Cp} \mathrm{p}^{\prime}=\mathrm{Cp} ; \quad 1^{\prime} \mathrm{M}=\mathrm{V}, \mathrm{Cp} \mathrm{p}^{\prime}=\mathrm{Cp}^{*} ; \quad 4 \mathrm{M}=\mathrm{Cr}, \mathrm{Cp}^{\prime}=\mathrm{Cp}^{*}$ $2 \mathrm{M}=\mathrm{Nb}, \mathrm{Cp^{ \prime }}=\mathrm{Cp} ; \mathbf{2}^{\prime} \mathrm{M}=\mathrm{Nb}, \mathrm{Cp^{ \prime }}=\mathrm{C} \mathrm{p}^{*} ; 5 \mathrm{M}=\mathrm{Mo}, \mathrm{Cp}^{\prime}=\mathrm{Cp}^{*}$ $3 \mathrm{M}=\mathrm{Ta}, \mathrm{Cp} \mathrm{p}^{\prime}=\mathrm{Cp} ; \mathbf{3}^{\prime} \mathrm{M}=\mathrm{Ta}, \mathrm{Cp}^{\prime}=\mathrm{Cp}^{*} ; \quad 6 \mathrm{M}=\mathrm{W}, \mathrm{Cp}^{\prime}=\mathrm{Cp}^{*}$

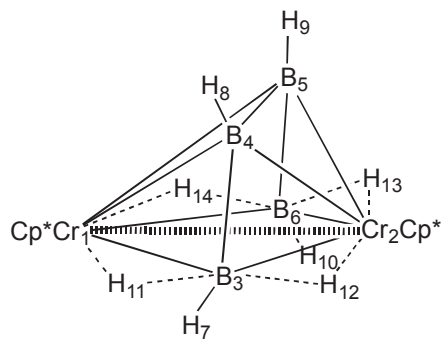

10

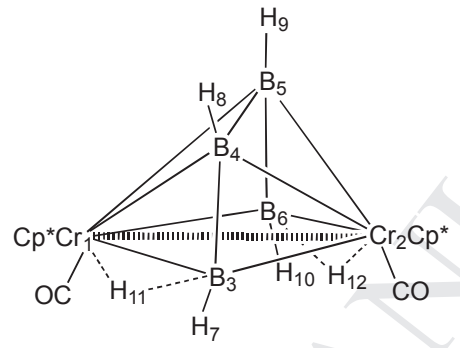

11

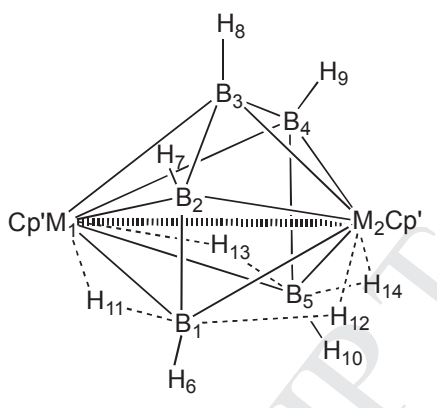

$7 \mathrm{M}=\mathrm{Cr}, \mathrm{Cp}^{\prime}=\mathrm{Cp}^{*}$

$8 \mathrm{M}=\mathrm{Mo}, C p^{\prime}=C p^{*}$

$9 \mathrm{M}=\mathrm{W}, \mathrm{Cp} \mathrm{p}^{\prime}=-\mathrm{Cp^{* }}$

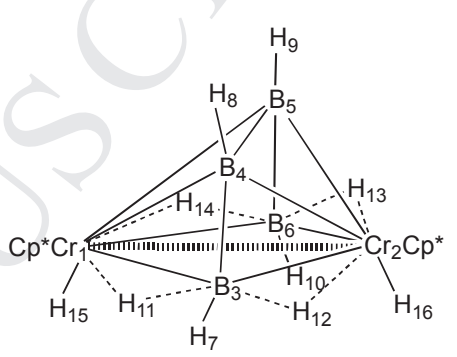

12

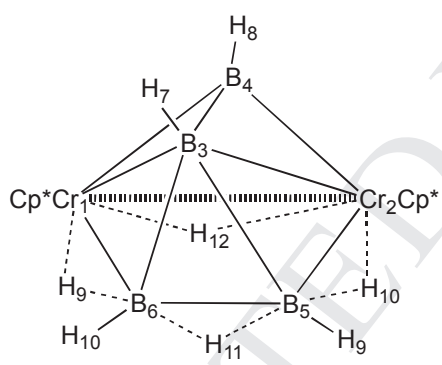

13

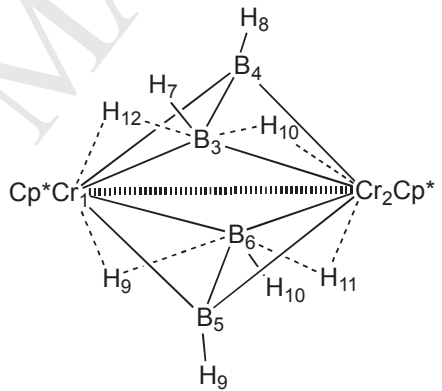

14

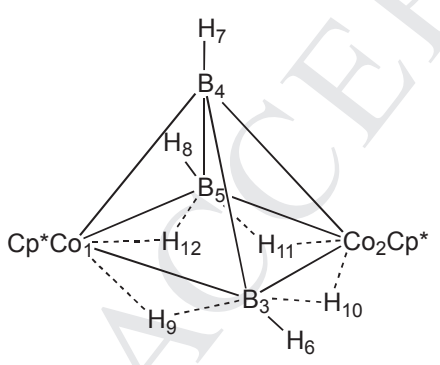

15

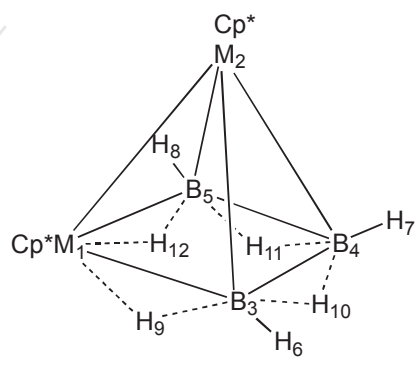

16 (M = Rh), 17 (M = Ir)

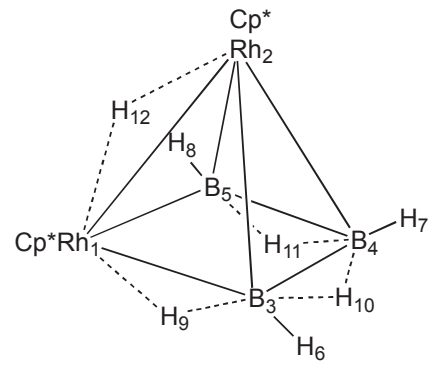

$16 b$

Chart 1. Examples of $\mathrm{M}_{2} \mathrm{~B}_{3}, \mathrm{M}_{2} \mathrm{~B}_{4}$ and $\mathrm{M}_{2} \mathrm{~B}_{5}$ dimetallaborane cluster compounds. 


\section{Computational details}

Density functional theory calculations were carried out using the Amsterdam Density Functional (ADF) program [36] developed by Baerends and co-workers [37]. The Vosko-WilkNusair parameterization [38] was used for the local density approximation (LDA) with gradient corrections for exchange (Becke88) [39,40] and correlation (Perdew86) [41]. The geometry optimization procedure was based on the method developed by Versluis and Ziegler [42]. Relativistic corrections were added using the ZORA (zeroth order regular approximation) scalar Hamiltonian [43-45]. Structures were optimized using an all-electron TZ2P basis sets [36], available in the ADF program without any geometrical constraint. Experimental geometries were taken as inputs for compounds when they were available. The nature of the stationary points after optimization was checked by calculations of the harmonic vibrational frequencies to ensure that genuine minima were obtained.

The BP86/TZ2P optimized geometries were then used as inputs for the NMR computations using all-electron TZ2P basis sets [36,38] at the relativistic scalar (SC) ZORA level of theory. NMR chemical shifts were also calculated with the hybrid Becke-Lee-Yang-Parr (B3LYP) functional [46-48] for comparison, using the BP86/TZ2P optimized geometries. The computation of the NMR shielding tensors employed gauge-including atomic orbitals (GIAOs) [49-52], using the implementation of Schreckenbach, Wolff, Ziegler, and co-workers [53-57]. NMR calculations were performed including the spin-orbit (SO) term with both BP86 and B3LYP functionals. TMS $\left(\mathrm{SiMe}_{4}\right)$ was used as an internal standard for the ${ }^{1} \mathrm{H}$ NMR. The projected ${ }^{11} \mathrm{~B}$ chemical shielding values, determined from relativistic scalar ZORA calculations were referenced to $\mathrm{B}_{2} \mathrm{H}_{6}$ as the primary reference point, and these chemical shift values $(\delta)$ were then converted to the standard $\mathrm{BF}_{3} \bullet \mathrm{OEt}_{2}$ scale using the experimental value of $+16.6 \mathrm{ppm}$ for $\mathrm{B}_{2} \mathrm{H}_{6}$. 


\section{Results and discussion}

\section{Geometries}

Precise structural arrangements are necessary for getting accurate ${ }^{11} \mathrm{~B}$ and ${ }^{1} \mathrm{H}$ NMR chemical shifts [58]. Different $\mathrm{M}_{2} \mathrm{~B}_{3}, \mathrm{M}_{2} \mathrm{~B}_{4}$ and $\mathrm{M}_{2} \mathrm{~B}_{5}$ clusters were then geometrically optimized and compared to experimental data where available. We commence with the $\left(\mathrm{Cp}{ }^{\prime} \mathrm{M}\right)_{2}\left(\mathrm{~B}_{2} \mathrm{H}_{6}\right)_{2}$ cluster compounds $\left(\mathrm{Cp}^{\prime}=\mathrm{C}_{5} \mathrm{H}_{5}(\mathrm{Cp})\right.$ or $\mathrm{C}_{5} \mathrm{Me}_{5}\left(\mathrm{Cp}^{*}\right) ; \mathrm{M}=\mathrm{V}, \mathrm{Nb}$, Ta). Salient optimized geometrical parameters are collected in Table 1. The three representatives with Group 5 metals were characterized crystallographically and spectroscopically with either $\mathrm{Cp}$ in the cases of $(\mathrm{CpV})_{2}\left(\mathrm{~B}_{2} \mathrm{H}_{6}\right)_{2}(\mathbf{1})[59]$ and $(\mathrm{CpNb})_{2}\left(\mathrm{~B}_{2} \mathrm{H}_{6}\right)_{2}(\mathbf{2})$ [59] or $\mathrm{Cp}^{*}$ in the case of $(\mathrm{Cp} * \mathrm{Ta})_{2}\left(\mathrm{~B}_{2} \mathrm{H}_{6}\right)_{2}\left(\mathbf{3}^{\prime}\right)$ [59]. Overall, the optimized bond lengths reproduce the X-ray data rather well, within a few hundredths of $\AA$, a degree of agreement which is typical for the DFT level employed. The computed M-M bond distances of $2.735 \AA, 2.954 \AA$, and $2.941 \AA$ are comparable to the corresponding X-ray values of $2.787(2) \AA, 2.948(16) \AA$, and 2.933(4) $\AA$, respectively, for clusters 1, 2 and 3'. The average M-B bond lengths in these compounds of $2.269 \AA, 2.405 \AA$, and $2.387 \AA$ are substantially longer than the sum of the $\mathrm{M}$ and $\mathrm{B}$ covalent radii, i.e., $2.20 \AA$, $2.22 \AA$, and $2.22 \AA$ for $\mathrm{V}-\mathrm{B}, \mathrm{Nb}-\mathrm{B}$, and Ta-B, respectively [60], supporting the presence of hydrogen atoms bridging M-B bonds. Comparing the computed and experimental B-B bond lengths indicates that the computed ones are $0.02 \AA$ longer for $\mathbf{1}$ and 3', but $0.04 \AA$ shorter for $\mathbf{2}$. Finally, we note that geometries computed either with $\mathrm{Cp}$ or $\mathrm{Cp}^{*}$ as ancillary ligands attached to the metal atoms are highly similar.

For the Group-6 metal triad, only the molecular structures of $(\mathrm{Cp} * \mathrm{Mo})_{2}\left(\mathrm{~B}_{2} \mathrm{H}_{6}\right)_{2}(5)$ and $(\mathrm{Cp} * \mathrm{~W})_{2}\left(\mathrm{~B}_{2} \mathrm{H}_{6}\right)_{2}(6)$ were proposed with an arrangement similar to that encountered for the 
Group-5 metal triad discussed above, on the basis of the ${ }^{11} \mathrm{~B},{ }^{1} \mathrm{H}$ NMR and mass spectrometry details $[19,20]$. They were computed as well as the hypothetical $\mathrm{Cr}$ homolog $(\mathrm{Cp} * \mathrm{Cr})_{2}\left(\mathrm{~B}_{2} \mathrm{H}_{6}\right)_{2}$ (4). BP86/TZ2P optimized geometries show M-M bond lengths of $2.760 \AA, 2.900 \AA$, and 2.909 $\AA$ for compounds $\mathbf{4 , 5}$, and $\mathbf{6}$, respectively (Table 1 ). The latter for instance is notably longer than the W-W bond length of $2.8174(8) \AA$ reported for $\left(\mathrm{Cp}^{*} \mathrm{~W}\right)_{2} \mathrm{~B}_{5} \mathrm{H}_{9}$ for instance $[19,20]$, but still short enough to indicate some significant metal-metal bonding interactions at first sight. The rather long computed average M-B bond lengths $(2.4015 \AA$ for W-B for instance compared to $2.239 \AA$ in $(\mathrm{Cp} * \mathrm{~W})_{2} \mathrm{~B}_{5} \mathrm{H}_{9}$, vide infra) may be due to the presence of eight bridging hydrogen atoms. The B-B bond lengths in compounds 4, 5 and $\mathbf{6}$ are considerably shorter $(1.671 \AA$, 1.690 $\AA, 1.686 \AA$ ) than normal B-B single bonds observed for the related compounds 1-3 (ca.1.75 $\AA$ ), reflecting strong B-B bonding interactions in these clusters.

For the oblato-nido clusters $(\mathrm{Cp} * \mathrm{M})_{2} \mathrm{~B}_{5} \mathrm{H}_{9}(\mathrm{M}=\mathrm{Cr}(7)$, Mo (8), W (9)), the core of which depicts a hexagonal bipyramid with one missing equatorial vertex, the BP86/TZ2P/SC optimized geometries are in good agreement with the metrical parameters measured experimentally by Xray crystallography (see Table ST1, Supporting Information). The computed M-M bond distances of $2.592 \AA, 2.810 \AA$ and $2.835 \AA$ nicely reproduce the experimental ones which are 2.625(9) $\AA[15], 2.8085(6) \AA[30]$, and 2.817(8) $\AA[19,20]$ for 7, 8, and 9, respectively. They indicate significant metal-metal bonding interactions in these clusters.

As said above, the $\mathrm{Cr}$ compound $(\mathrm{Cp} * \mathrm{Cr})_{2}\left(\mathrm{~B}_{2} \mathrm{H}_{6}\right)_{2}(4)$ has not been reported so far. With a $\mathrm{Cr}_{2} \mathrm{~B}_{4}$ core, the compound $\left(\mathrm{Cp}^{*} \mathrm{Cr}\right)_{2} \mathrm{~B}_{4} \mathrm{H}_{8}(\mathbf{1 0})$ has been characterized instead with a nido pentagonal bipyramidal cage with one missing equatorial vertex and the two $\mathrm{Cr}$ atoms occupying the axial positions and separated by $2.870(2) \AA$ (Chart 1) [61]. The reaction of $\mathbf{1 0}$ with CO yielded $(\mathrm{Cp} * \mathrm{Cr})_{2} \mathrm{~B}_{4} \mathrm{H}_{6}(\mathrm{CO})_{2}(\mathbf{1 1}$, Chart 1) as the major product [14]. The same nido pentagonal 
bipyramidal $\mathrm{Cr}_{2} \mathrm{~B}_{4}$ cage was confirmed by an X-ray analysis with a $\mathrm{Cr}-\mathrm{Cr}$ bond length 2.792(1) $\AA$ [14]. A DFT geometry optimization of $\mathbf{1 0}$ at the BP86/TZ2P/SC all-electron scalar ZORA level resulted in a structure with a Cr1-Cr2 bond length abnormally short of $2.548 \AA$ (Table 2), suggesting that the chemical formula of $\mathbf{1 0}$ reported experimentally might be wrong (hydrogen atoms were not directly located from the X-ray analysis [14]). Due to the large difference with the experimental value $(0.322 \AA)$, two hydrogen atoms bridging the Cr1-B4 and Cr2-B5 vectors were added (see $\mathbf{1 0}$ in Chart 1). The optimized structure resulted in the B3-B4 bond breakage. The two hydrogen atoms were then terminally added to two $\mathrm{Cr}$ atoms analogously to the $\mathrm{CO}$ ligand in 11. Interestingly, the optimized geometry of this model structure $(\mathrm{Cp} * \mathrm{Cr})_{2} \mathrm{~B}_{4} \mathrm{H}_{10}$ (see 12 in Chart 1) revealed a $\mathrm{Cr}_{2} \mathrm{~B}_{4}$ open cage similar to that computed for $\mathbf{1 0}$ but with a $\mathrm{Cr} 1-\mathrm{Cr} 2$ bond length considerably longer of $2.7356 \AA$, still $0.13 \AA$ shorter than the experimental value experimentally measured for $(\mathrm{Cp} * \mathrm{Cr})_{2} \mathrm{~B}_{4} \mathrm{H}_{8}$ (10) but relatively close to the $\mathrm{Cr}-\mathrm{Cr}$ bond length experimentally observed in $\left(\mathrm{Cp} * \mathrm{Cr}_{2}\right)_{2} \mathrm{~B}_{4} \mathrm{H}_{6}(\mathrm{CO})_{2}(\mathbf{1 1}), 2.792(1) \AA$ which compares rather well to the computed distance of $2.764 \AA$ (Table 2). The comparison of the computed B-B distances in $\mathbf{1 0}$ and $\mathbf{1 2}$ with those reported experimentally, are not much informative about the 'right' formula. The B3-B4, B4-B5, B5-B6 distances of $\mathbf{1 0}$ are $1.735,1.637,1.725 \AA$, respectively, whereas those in the model compound $\left(\mathrm{Cp}^{*} \mathrm{Cr}\right)_{2} \mathrm{~B}_{4} \mathrm{H}_{10}$ 12, are $1.701,1.639,1.702 \AA$, respectively. They all deviate somewhat from the corresponding experimental (not very precise) X-ray values which are 1.75(3), 1.75(3), 1.61(3) $\AA$, respectively. Notably, the Cp* ligands are not parallel but tilted away from the open face of the cluster cage with angles of $28.1(8)^{\circ}$ and $30.4(20)^{\circ}$ experimentally measured for 10 and $\mathbf{1 1}$, respectively $[14,61,63]$. Interestingly, the $\mathrm{Cp}^{*}$ rings are hardly tilted in the optimized geometry of $\mathbf{1 0}$ but significantly tilted in $\mathbf{1 1}$ and $\mathbf{1 2}\left(16^{\circ}\right.$ and $14^{\circ}$, respectively). Additional structural arrangements can be envisaged for this compound, 
such as a capped square pyramidal $(\mathbf{1 3})$ or $(\mathrm{Cp} * \mathrm{Cr})_{2}\left(\mathrm{~B}_{2} \mathrm{H}_{4}\right)_{2}(\mathbf{1 4})$ structures with symmetrical $\left(\mathrm{B}_{2} \mathrm{H}_{4}\right)$ units (Chart 1) [61]. Their geometry optimization results in $\mathrm{Cr}-\mathrm{Cr}$ bond lengths of 2.22 and $2.42 \AA$, respectively. That in the former is comparable to the $\mathrm{Cr}-\mathrm{Cr}$ triple bond of $2.200(3)$ encountered in $\mathrm{Cp}_{2} \mathrm{Cr}_{2}(\mathrm{CO})_{4}[64]$ for instance.

The late-transition metal borane compounds of formula $\left(\mathrm{Cp}^{*} \mathrm{M}\right)_{2} \mathrm{~B}_{3} \mathrm{H}_{7}$ have been crystallographically characterized for $\mathrm{M}=\mathrm{Co}$ (15) [5] and $\mathrm{Rh}$ (16) [7] (Chart 1). They display a square pyramidal geometrical cage with two metal atoms occupying basal positions in the case of 15 (nido-2,4-( $\left.\left(\mathrm{Cp}^{*} \mathrm{Co}\right)_{2} \mathrm{~B}_{3} \mathrm{H}_{7}\right)$ and one metal atom occupying the apical position and the second metal atom occupying one basal position in the case of 16 (nido-1,2-(Cp $\left.{ }^{*} \mathrm{Rh}\right)_{2} \mathrm{~B}_{3} \mathrm{H}_{7}$ ). Interestingly, there is an M-M bond in the latter but not in the former. A tautomer of 16, i.e., nido-1,2-( $\left.\mathrm{Cp}^{*} \mathrm{Rh}\right)_{2}(\mu-\mathrm{H}) \mathrm{B}_{3} \mathrm{H}_{6}(\mathbf{1 6 b})$ was also spectroscopically observed [7]. The optimized distances are in a rather good agreement with the experimentally available X-ray values (Table 2, Table ST3, Supporting Information). The computed Rh-Rh bond length in $\mathbf{1 6}$ is $2.740 \AA, 0.05 \AA$ larger than the X-ray value of $2.6892(3) \AA[7]$. This deviation might be attributed to the presence of two independent molecules in the unit cell, with a disordered $\mathrm{Cp}^{*}$ ring in one molecule, and the fully disordered second molecule, observed in the solid state X-ray structure [7]. This distance is notably shorter than the Rh-Rh bond length of $2.8478(11) \AA$ reported for the nido-2,4-( $\left.\mathrm{Cp}^{*} \mathrm{Rh}\right){ }_{2} \mathrm{~B}_{3} \mathrm{H}_{6} \mathrm{Cl}$ cluster for which the two rhodium atoms occupy two adjacent basal positions [7]. Geometry optimization of $\mathbf{1 6} \mathbf{b}$ results in an intermediate $\mathrm{Rh}-\mathrm{Rh}$ bond length of $2.744 \AA$. A very small energy difference, $0.26 \mathrm{kcal} / \mathrm{mol}$, is computed between tautomers 16 and 16b in favor of the former. The iridium analogue $\left(\mathrm{Cp}^{*} \mathrm{Ir}\right)_{2} \mathrm{~B}_{3} \mathrm{H}_{7}(\mathbf{1 7})$ has not been isolated until now. BP86/TZ2P metrical parameters computed with the same arrangement as its $\mathrm{Rh}$ congener $\mathbf{1 6}$ are comparable to those measured in related diiridium cluster compounds [8]. For example, 
the computed Ir-Ir bond length of $2.7733 \AA$ is somewhat shorter than that in arachno$(\mathrm{Cp} * \mathrm{IrH})_{2}(\mu-\mathrm{H}) \mathrm{B}_{2} \mathrm{H}_{5}$ which is 2.8227(8) $\AA$ [8] but similar to the value of 2.7814(9) $\AA$ measured in arachno- $(\mathrm{Cp} * \mathrm{IrH})_{2} \mathrm{~B}_{4} \mathrm{H}_{8}[8]$.

\section{Electron counts and electronic structures}

Dimetallaboranes of the earlier transition metals often present interesting challenges to the well-established cluster electron-counting rules, the so-called Polyhedral Skeletal Electron Pair Theory (PSEPT) [65,70], due to their distinctly oblate (flattened along the M-M cross-cluster axis) rather than closely spherical nature $[1,13,71,72]$. They are generally characterized with short metal-metal cross-cluster distances and apparent formal cluster electron counts a few skeletal electron pairs (sep) less (generally three) than required for canonical closo- or nidostructure of the same nuclearity. Previous DFT calculations suggested that these oblato arrangements are indeed very stable compounds, due to an intricate mutual interaction of the dimetal fragment and the borane cage. Indeed, some of us showed that bringing two CpM or $\mathrm{Cp} * \mathrm{M}$ fragments close together generates a set of three frontier metal orbitals that can only interact with the frontier orbitals of the borane fragment. These orbitals on each of these complementary fragments, which normally would be filled in a late transition-metal metallaborane with a spherical deltahedral shape, interact strongly to generate three low-lying filled orbitals and three high-lying unfilled orbitals. As a result, they generate a metal-metal cross-cluster bonding where the effective sep counts $(p+1$ (oblato-closo), $p+2$ (oblato-nido), $p$ +3 (oblato-arachno) if $p$ is the number of occupied vertices) is three seps larger than the apparent sep count obtained via the classical PSEPT $[1,13,73]$. 
If these PSEPT-extended rules are applied to compounds 1-3 and 1'-3', they can be viewed as oblato-arachno species with a structure derived from an 8-vertex oblato-closo hexagonal bipyramidal cluster encountered for the effective 9-sep cluster $\left(\mathrm{Cp}^{*} \mathrm{Re}\right)_{2} \mathrm{~B}_{6} \mathrm{H}_{4} \mathrm{Cl}_{2}$ [13], with two non-adjacent vacant sites. Indeed, they have the same effective sep count of $9([-2$ (Cp’M) x $2+$ $2(\mathrm{BH}) \times 4+1$ (bridging $\left.\left.\mathrm{H}) \times 8+6\left(t_{2 g} \mathrm{M}_{2}\right)\right] / 2\right)$, in agreement with a somewhat short metalmetal cross-cluster bond. Surprisingly enough, clusters 4-6 with the same oblato-arachno hexagonal bipyramidal core possess an effective sep count of $10([-1(\mathrm{Cp} * \mathrm{M}) \times 2+2(\mathrm{BH}) \times 4+$ 1 (bridging $\left.\left.\mathrm{H}) \times 8+6\left(t_{2 g} \mathrm{M}_{2}\right)\right] / 2\right)$, i.e., one more that the expected electron count. In the same manner, clusters 7-9 can be viewed as oblato-nido $\mathrm{M}_{2} \mathrm{~B}_{5}$ species also derived from an 8-vertex closo hexagonal bipyramidal cluster, possessing the expected effective sep count of 9 ([-1 $\left(\mathrm{Cp}^{*} \mathrm{M}\right) \times 2+2(\mathrm{BH}) \times 5+1$ (bridging $\left.\left.\left.\mathrm{H}\right) \times 4+6\left(t_{2 g} \mathrm{M}_{2}\right)\right] / 2\right]$.

With only 8 hydrogen atoms cluster $\left(\mathrm{Cp}^{*} \mathrm{Cr}\right)_{2} \mathrm{~B}_{4} \mathrm{H}_{8}, \mathbf{1 0}$ possesses 8 sep's $\left[-1\left(\mathrm{Cp}^{*} \mathrm{Cr}\right)\right.$ x $2+2$ (BH) $x 4+1$ (bridging $\left.\mathrm{H}) \times 4+6\left(t_{2 g} \mathrm{M}_{2}\right)\right] / 2$ ), the expected count if it is assumed that the $\mathrm{Cr}_{2} \mathrm{~B}_{4}$ core depicts an open nido pentagonal bipyramidal cage. This implies that the carbonyl analog $\mathbf{1 1}$ which adopts the same polyhedral cage, has 9 sep's, i.e., one more than expected. A close examination of the X-ray structures of $\mathbf{1 0}$ and $\mathbf{1 1}$ and the optimized geometries of 10-12 (B3-B4B5 bond angles and non-bonded B3-B6 distance) indicates that in turn, $\mathbf{1 0}$ and $\mathbf{1 1}$ are better considered as oblato-arachno hexagonal bipyramidal clusters, deriving from an 8-vertex closo hexagonal bipyramid by removal of two adjacent basal vertices. Consequently, $\mathbf{1 0}$ is an electrondeficient species, whereas 11 (and $\mathbf{1 2}$ which possesses 10 hydrogen atoms) possess the expected count of sep's. Fehlner et al. already noted the electronic unsaturation of $\mathbf{1 0}$ on the basis of its reactivity and a molecular orbital analysis using semi-empirical Fenske-Hall calculations [63]. The model clusters 13 and 14 [61,62] also result in an effective sep count of five. The shortage of 
skeletal electrons in cluster $\mathbf{1 4}$ is compensated by a formal $\mathrm{Cr}-\mathrm{Cr}$ triple bond and thus, in some ways, related to the two-electron richer $(\mathrm{CpNbCO})_{2}(\mathrm{RCCR})_{2}$ compound $(\mathrm{M}=\mathrm{M}$ double bond $)$ as theoretically pointed out by Hoffmann and colls. [74].

Satisfactorily, all late-transition metal compounds $\mathbf{1 5 - 1 7}$ with a nido square pyramidal $\mathrm{M}_{2} \mathrm{~B}_{3}$ cage and a 7-sep count $([2(\mathrm{Cp} * \mathrm{M}) \times 2+2(\mathrm{BH}) \times 3+1$ (bridging $\mathrm{H}) \times 4] / 2)$ obey the classical PSEPT rules, as expected.

When dealing with electron counting, it is of major importance not to forget that all the rules, which govern the structure/electron count relationship, are based on the so-called the 'closedshell principle' $[1,73]$. In other words, these stable dimetallaboranes should exhibit a significant highest occupied molecular orbital (HOMO)-lowest unoccupied molecular orbital (LUMO) energy gap. Indeed, BP86/TZ2P/SC HOMO-LUMO gaps of ca. $2 \mathrm{eV}$ are computed for many of the studied compounds in agreement with their electron counts (Table ST4, Supporting Information). $(\mathrm{Cp} * \mathrm{Cr})_{2}\left(\mathrm{~B}_{2} \mathrm{H}_{6}\right)_{2}(4)$ has not been isolated yet and its heavier congeners 5 and $\mathbf{6}$ have only been characterized in solution $[19,20]$. They are too much electron-rich by one extra sep according to the PSEPT-extended rules (see above). Accordingly, a small HOMO-LUMO gap of only $0.71 \mathrm{eV}$ was computed for the non-reported species 4. Larger energy gaps of 1.42 and $1.51 \mathrm{eV}$ were computed for $\mathbf{5}$ and $\mathbf{6}$, respectively. For the 'unsaturated' cluster 10, a small HOMO-LUMO gap of only $0.65 \mathrm{eV}$, which does not militate for its existence, led us to look into the details of the structure. A HOMO-LUMO gap of $1.64 \mathrm{eV}$ is computed for $\mathbf{1 1}$ which has two electrons more. With two hydrogen atoms more, the HOMO-LUMO gap jumps to $2.21 \mathrm{eV}$ for model 12, isoelectronic to 11. Additionally, HOMO-LUMO gaps of 1.57 and $0.98 \mathrm{eV}$ are computed for the model compounds 13 and 14, respectively. Obviously, these results render the formula of $\mathbf{1 0}$ questionable. 


\section{NMR chemical shifts}

The number of direct metal-boron and boron-boron bonds, the presence or absence of bridging hydrogen atoms, the metal identity, the atomic coordination number, the atom charges are some parameters which may influence ${ }^{1} \mathrm{H}$ and ${ }^{11} \mathrm{~B}$ NMR chemical shifts in metallaboranes [58]. ${ }^{11} \mathrm{~B},{ }^{1} \mathrm{H}$ and ${ }^{13} \mathrm{C}$ NMR chemical shifts were calculated at the DFT level using both BP86 and hybrid B3LYP functionals, with and without scalar spin-orbit (SO) relativistic corrections, using BP86 optimized geometries. Data are collected in Tables 3-5 and Tables ST5 and ST6 (Supporting Information) together with the experimental values where available. It turns out overall that the $\mathrm{B} 3 \mathrm{LYP} / \mathrm{TZ2} / \mathrm{SO}$ ZORA calculated isotropic magnetic shielding constants (converted to ${ }^{11} \mathrm{~B},{ }^{13} \mathrm{C}$ and ${ }^{1} \mathrm{H}$ NMR chemical shifts) agree very well with experimental data for clusters with the second and third row early-transition metals $\mathrm{Nb}$, Ta, Mo and $\mathrm{W}$, whereas BP86/TZ2P/SC computed values are in a better agreement for the clusters containing the first row early-transition metals $\mathrm{V}$ and $\mathrm{Cr}$. The importance of both scalar and spin-orbit relativistic corrections for the shielding tensors in heavy transition metal complexes was observed previously by Autschbach et al. [75]. Bridging hydrogen atoms in these clusters are relatively shielded and resonate around -6 to $-12 \mathrm{ppm}$.

A single ${ }^{11} \mathrm{~B}$ NMR resonance is experimentally observed at $1.7 \mathrm{ppm}$ for $(\mathrm{CpV})_{2}\left(\mathrm{~B}_{2} \mathrm{H}_{6}\right)_{2}(\mathbf{1})$ and $(\mathrm{CpNb})_{2}\left(\mathrm{~B}_{2} \mathrm{H}_{6}\right)_{2}(2)$ and at $-4.0 \mathrm{ppm}$ for $\left(\mathrm{Cp}^{*} \mathrm{Ta}\right)_{2}\left(\mathrm{~B}_{2} \mathrm{H}_{6}\right)_{2}\left(\mathbf{3}^{\prime}\right)$, revealing the symmetrical nature of these structures in solution [59]. DFT calculations also predict the symmetrical nature

of these clusters. In the case of 1 , the ${ }^{11} \mathrm{~B}$ NMR chemical shifts computed at the BP86/TZ2P and B3LYP/TZ2P levels deviate downfield from the experimental values only by $4 \mathrm{ppm}$ for the former but $17.5 \mathrm{ppm}$ for the latter (Table 3). For the carbon atoms of the $\mathrm{Cp}$ ligands, a reverse 
situation, but less pronounced is computed with a maximum deviation of $6 \mathrm{ppm}$ upfield at the BP86/TZ2P/SC level and only $1 \mathrm{ppm}$ at the B3LYP/TZ2P/SC level when compared to the experimental ${ }^{13} \mathrm{C}$ NMR chemical shifts. On the other hand, both BP86/TZ2P/SC and B3LYP/TZ2P/SC computed ${ }^{1} \mathrm{H}$ NMR chemical shift values are in good agreement with the experimental ones with a maximum deviation of $1 \mathrm{ppm}$. For 2 , the more accurate ${ }^{11} \mathrm{~B}$ NMR chemical shifts are computed at the B3LYP/TZ2P/SO level (only slightly better than without SO inclusion) with a maximum deviation of only $0.5 \mathrm{ppm}$ downfield whereas the BP86/TZ2P/SO computed values deviate by 2 ppm upfield. For the ${ }^{13} \mathrm{C}$ NMR, the maximum deviation obtained at the $\mathrm{B} 3 \mathrm{LYP} / \mathrm{TZ2P} / \mathrm{SO}$ and $\mathrm{BP} 86 / \mathrm{TZ2P} / \mathrm{SO}$ levels are $6 \mathrm{ppm}$ and $2.5 \mathrm{ppm}$ downfield, respectively. Both B3LYP and BP86 methods are successful in predicting the ${ }^{1} \mathrm{H}$ NMR values with a maximum deviation of $1.5 \mathrm{ppm}$. In the case of the tantalum cluster 3 ', ${ }^{11} \mathrm{~B}$ NMR chemical shifts computed at the B3LYP/TZ2P level including SO contributions result in a good agreement with the experimentally observed values with a maximum deviation of $3 \mathrm{ppm}$ upfield ( $7 \mathrm{ppm}$ at the BP86/TZ2P/SO level). We note that the inclusion of the SO term increases considerably the accuracy. The ${ }^{13} \mathrm{C}$ NMR chemical shift values are computed with a maximum deviation of 3 $\mathrm{ppm}$ at the $\mathrm{BP} 86 / \mathrm{TZ2P} / \mathrm{SC}$ level and $6 \mathrm{ppm}$ at the $\mathrm{B} 3 \mathrm{LYP} / \mathrm{TZ2P} / \mathrm{SC}$ level. Though the inclusion of SO term has a considerable effect on ${ }^{11} \mathrm{~B}$ NMR chemical shifts, only a small effect ( $\left.0.3 \mathrm{ppm}\right)$ is observed for the chemical shifts of the methyl and cyclopentadienyl carbon atoms. The computed ${ }^{1} \mathrm{H}$ NMR chemical shifts for the terminal and bridging hydrogen atoms are comparable at both B3LYP/TZ2P/SO and BP86/TZ2P/SO levels with deviations of 1-2 ppm.

When comparing compounds $\mathbf{1}, \mathbf{2}$, and $\mathbf{3}$, i.e., from $\mathrm{V}$ to $\mathrm{Ta}$, the boron atoms and the bridging hydrogen atoms experience slightly more shielding but the reverse situation is observed for terminal hydrogen atoms. A look at the individual $\mathrm{B} 3 \mathrm{LYP} / \mathrm{TZ2} / \mathrm{P} O \mathrm{SO}$ computed components 
$\sigma_{(\mathrm{dia})}, \sigma_{(\text {para })}$, and $\sigma_{(\mathrm{so})}$ of the shielding tensors indicates that the diamagnetic shielding largely dominates in all the cases as expected and is comparable in all clusters (Fig. 1). On the other hand, the paramagnetic shielding decreases considerably while going from $\mathrm{V}$ to $\mathrm{Ta}$, from -105 ppm to $-71 \mathrm{ppm}$. Not surprisingly, the shielding from the relativistic spin-orbit term strongly increases from -0.3 for $\mathrm{V}$ and $\mathrm{Nb}$ to $-7.0 \mathrm{ppm}$ for $\mathrm{Ta}$. In fact, the large low-field shifts, which are characteristics of the effect of the neighboring transition metals on ${ }^{11} \mathrm{~B}$ NMR resonances, were already ascribed to the paramagnetic term by Fehlner et al. [34].

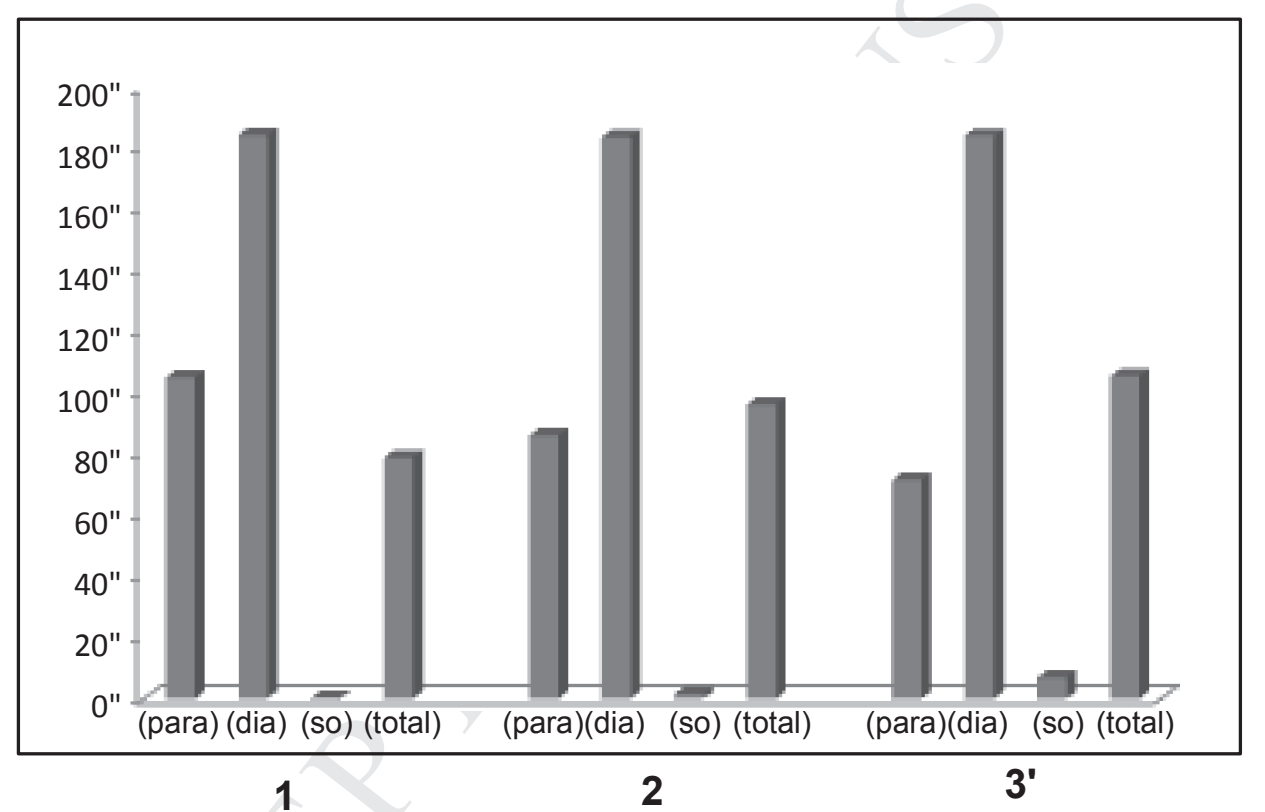

Fig. 1. Individual components of shielding tensors (ppm) $\sigma_{(\text {para })}, \sigma_{(\mathrm{dia})}$ and $\sigma_{(\mathrm{so})}$ Vs. total shielding in ${ }^{11}$ B NMR of compounds 1, 2, and $\mathbf{3}^{\prime} \cdot \sigma_{(\text {para })}$ and $\sigma_{(\mathrm{so})}$ are plotted as $\left|\sigma_{(\text {para })}\right|$ and $\left|\sigma_{(\text {so })}\right|$.

For the metallaborane clusters $(\mathrm{Cp} * \mathrm{Mo})_{2}\left(\mathrm{~B}_{2} \mathrm{H}_{6}\right)_{2}(\mathbf{5})$ and $(\mathrm{Cp} * \mathrm{~W})_{2}\left(\mathrm{~B}_{2} \mathrm{H}_{6}\right)_{2}(\mathbf{6})$, a single signal in the ${ }^{11} \mathrm{~B}$ NMR is also experimentally observed supporting the highly symmetric nature of these clusters in solution. For $\mathbf{5}$ a signal is observed experimentally at $-58.6 \mathrm{ppm}$ in the low frequency region. With the BP86 functional, deviations up to $6 \mathrm{ppm}$ (without SO) or $7 \mathrm{ppm}$ (with SO) in 
the upfield region are calculated (Table 4). Maximum deviations of upfield 2 ppm (without SO) and 4 ppm (with SO) are computed with the B3LYP functional. More surprisingly, the ${ }^{11} \mathrm{~B}$ NMR signal computed for 6 compares moderately to that experimentally measured at $-53.9 \mathrm{ppm}$. The closest computed value, which deviates by $10 \mathrm{ppm}$, is obtained at the B3LYP level (without SO). A good agreement between theory and experiment (0-2 ppm deviation) is observed for the ${ }^{1} \mathrm{H}$ NMR chemical shifts of the terminal and bridging hydrogen atoms with both methods (without $\mathrm{SO})$.

NMR data measured experimentally suggest that the electron-deficient cluster $(\mathrm{Cp} * \mathrm{Cr})_{2} \mathrm{~B}_{4} \mathrm{H}_{8}$, 10, is diamagnetic [61]. Two distinct boron environments are expected from a doublet signal at $126.5 \mathrm{ppm}$ and a multiplet signal at $34.3 \mathrm{ppm}$ (Table 5). Interestingly, the BP86/TZ2P computed values of $15.9,22.4,22.2$, and 16.6 ppm for B3, B4, B5, and B6, respectively, strongly differ, especially those for B4 and B5. With two hydrogen atoms more, the corresponding values change to $36.6,32.4,33.6$, and $37.2 \mathrm{ppm}$. A good agreement with the experimental values is then observed for B3 and B6, but again not for the experimentally unusually shielded B4 and B5 atoms. These results suggest that compound $\mathbf{1 0}$ should probably be corrected into $(\mathrm{Cp} * \mathrm{Cr})_{2} \mathrm{~B}_{4} \mathrm{H}_{10}, \mathbf{1 2}$, and that the experimental boron chemical shifts of $126.5 \mathrm{ppm}$ have been wrongly attributed to B4 and B5 atoms. Concerning, the ${ }^{1} \mathrm{H}$ NMR chemical shifts, the best agreement is observed between values experimentally measured for $\mathbf{1 0}$ and values theoretically computed for 12. For comparison, the ${ }^{11} \mathrm{~B}$ NMR chemical shifts were computed for the cluster $(\mathrm{Cp} * \mathrm{Cr})_{2} \mathrm{~B}_{4} \mathrm{H}_{6}(\mathrm{CO})_{2}(\mathbf{1 1})$ for which the two corresponding signals are experimentally observed at 63.9 and $34.9 \mathrm{ppm}$. A good agreement is found at either at BP86 or B3LYP levels of theory (Table 5). 
${ }^{11}$ B NMR chemical shifts of the two types of boron atoms (B2, B3 and B4) directly bonded to the metal atoms in $(\mathrm{Cp} * \mathrm{M})_{2} \mathrm{~B}_{5} \mathrm{H}_{9}(\mathrm{M}=\mathrm{Cr}(7), \mathrm{Mo}(\mathbf{8}), \mathrm{W}(9))$ experience a large systematic shift to higher field going from $\mathrm{Cr}$ to Mo to W, whereas the shift at upper field for the boron atoms (B1, B5) connected to the metal atoms via M-H-B bridged bonds are invariant (Table ST5 (Supporting Information) and Fig. 2). This trend is also predicted by the DFT calculations, although the agreement between computed and experimental values is far to be satisfactory. For 7 the better chemical shifts are computed at the BP86/TZ2P/SC level with a deviation of $3.5 \mathrm{ppm}$ for B1/B5, but 13 ppm for B2/B4 and 18 ppm for B3 downfield. Except for B3 for which a deviation of $9 \mathrm{ppm}$ is observed, the computed B3LYP/TZ2P/SC values differ by more than 20 ppm downfield. On the other hand, B3LYP/TZ2P/SC computed values for the Mo analogue 8 and $\mathrm{B} 3 \mathrm{LYP} / \mathrm{TZ2} / \mathrm{PO}$ computed values for the $\mathrm{W}$ analogue 9 are in a very good agreement with the experimental values with a maximum deviation of 3 ppm downfield (Table 4 and Fig. 2). Inclusion of relativistic spin-orbit corrections for the latter improves the B3LYP/TZ2P/SC computed ${ }^{11} \mathrm{~B}$ NMR chemical shifts considerably by ca. 7 ppm. Both B3LYP and BP86 computed ${ }^{1} \mathrm{H}$ NMR $\delta$ values for the B-H terminal and M-H-B bridging hydrogen atoms in close agreement with the experimental values with a maximum deviation of ca. $1 \mathrm{ppm}$.

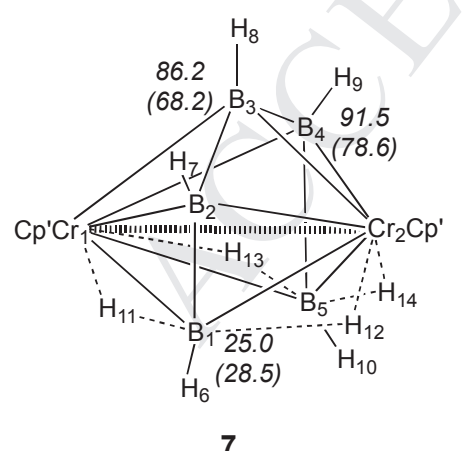

7

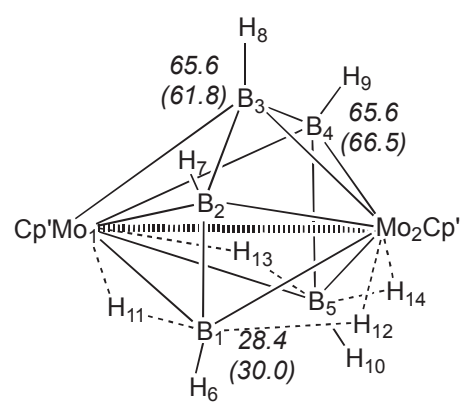

8

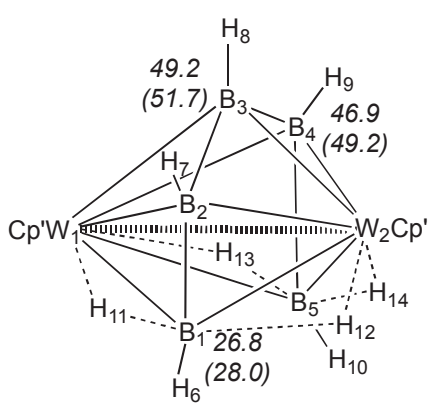

9

Fig. 2. Experimental and most accurate computed (in brackets) ${ }^{11} \mathrm{~B}$ NMR chemical shifts (ppm) for compounds 7-9 (BP86/TZ2P/SC for 7, B3LYP/TZ2P/SC for 8 , and B3LYP/TZ2P/SO for 9). 
${ }^{11} \mathrm{~B}$ NMR chemical shifts were also computed for the late transition metal borane compounds in order to also check the suitability of the computational method used (Table ST6 and Fig. 3). Experimentally, the nido-2,4-( $\left.\mathrm{Cp}^{*} \mathrm{Co}\right)_{2} \mathrm{~B}_{3} \mathrm{H}_{7}$ compound 15 exhibits two ${ }^{11} \mathrm{~B}$ NMR signals in a 2:1 ratio at $65.8 \mathrm{ppm}$ and $-18.1 \mathrm{ppm}$ [5]. Correct values (Table 10) with deviations of a few ppm are computed at the $\mathrm{BP} 86 / \mathrm{TZ} 2 \mathrm{P} / \mathrm{SC}, \mathrm{BP} 86 / \mathrm{TZ2P} / \mathrm{SO}$, and $\mathrm{B} 3 \mathrm{LYP} / \mathrm{TZ} 2 \mathrm{P} / \mathrm{SC}$ levels (the latter is slightly better). Good values are also computed for the ${ }^{1} \mathrm{H}$ NMR chemical shifts, especially at the B3LYP/TZ2P/SC level with maximum deviations of ca. $1 \mathrm{ppm}$. The signals of the two terminal hydrogen atoms $\mathrm{H} 6$ and $\mathrm{H} 8$, not attributed experimentally are computed to resonate around 0 ppm, justifying the possibility of overlapping experimentally with the H peak of TMS [5] and the strength of the computational methods used. For the nido-1,2-(Cp* Rh $)_{2} \mathrm{~B}_{3} \mathrm{H}_{7}$ species 16, the experimental ${ }^{11} \mathrm{~B}$ NMR spectrum results with four broad signals at 3.1, 5.8, 8.2, and $11.1 \mathrm{ppm}$ and a very broad signal at $21.1 \mathrm{ppm}$ [7]. Since only three signals are expected, these five signals must be due to the presence of two tautomers (16 and $\mathbf{1 6 b}$ for instance, see above) or even other additional species in solution, as confirmed later with variable-temperature NMR [7]. Indeed, calculations seem to indicate a more complex situation. Although, the different levels of calculations lead to somewhat different values (up to $10 \mathrm{ppm}$ difference), they show the same trend with the three boron atoms in $\mathbf{1 6}$ resonating in the same range around 0-10 ppm (Table 5 and Fig. 3), and the three boron atoms in $\mathbf{1 6 b}$ resonating strongly differently around -12 (B3), 1 (B4) and 48 (B5) ppm. With no experimental peaks measured at such low or high frequencies, we might conclude that $\mathbf{1 6 b}$ is not present in the solution of $\mathbf{1 6}$ and that the additional peak such as at $21 \mathrm{ppm}$ are due to other species. Interestingly, the ${ }^{11} \mathrm{~B}$ NMR peaks for the chlorineanalogue cluster $(\mathrm{Cp} * \mathrm{Rh})_{2} \mathrm{~B}_{3} \mathrm{H}_{6} \mathrm{Cl}$ show experimental ${ }^{11} \mathrm{~B}$ NMR chemical shifts at 48.0, 4.0, and $-5.3 \mathrm{ppm}$ for the three boron atoms [11]. 


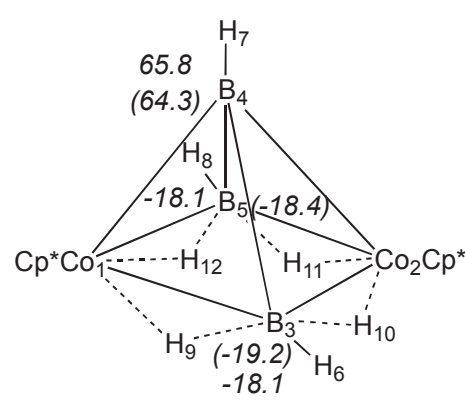

15

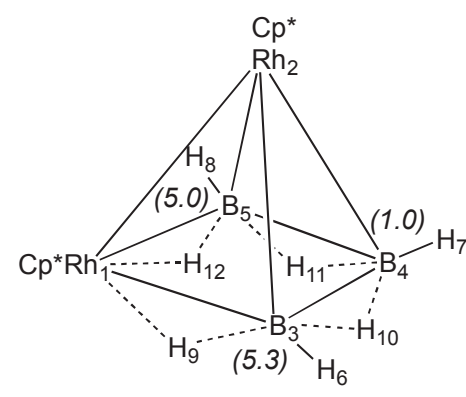

16

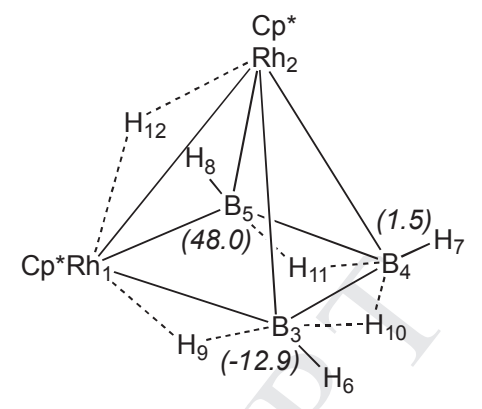

$16 b$

Fig. 3. DFT computed chemical shift values (ppm, in brackets) for clusters $\mathbf{1 5}$ $(\mathrm{B} 3 \mathrm{LYP} / \mathrm{TZ2P} / \mathrm{SC})$ and 16, 16b $(\mathrm{B} 3 \mathrm{LYP} / \mathrm{TZ2P} / \mathrm{SO})$. Experimental values for $\mathbf{1 5}$ are given for comparison.

\section{Conclusion}

The present study proves the strength of DFT methods in analyzing the electronic and magnetic properties of a series of dimetallaboranes with both early- and late-transition metals. In particular, the observed ${ }^{1} \mathrm{H}$ and ${ }^{11} \mathrm{~B}$ chemical shifts of most of the studied compounds can be reproduced with a rather good accuracy of a few ppm at the DFT-GIAO BP86/TZ2P/SC level for the compounds with first-row transition metal elements and at the B3LYP/TZ2P/SO level for those with second- and third-row transition metal elements. This shows that the quality of DFT computed ${ }^{1} \mathrm{H}$ and ${ }^{11} \mathrm{~B}$ chemical shifts generally attained for boranes and carboranes does not degrade much in the presence of transition metals, suggesting that any metallaborane is amenable to relatively accurate ${ }^{1} \mathrm{H}$ and ${ }^{11} \mathrm{~B}$ NMR computations. Indeed, the accuracy is sufficient for structural applications to elucidate the number and the location of bridging hydrogen atoms in experimentally poorly characterized metallaboranes such as $(\mathrm{Cp} * \mathrm{Cr})_{2} \mathrm{~B}_{4} \mathrm{H}_{8}$. 


\section{Acknowledgments}

The authors acknowledge the Indo-French Centre for Promotion of Advanced Research (IFCPAR) (Project No. 4405-1) for generous financial support. Dr. B. Le Guennic (Rennes) is thanked for helpful discussions.

\section{Appendix. Supplementary data}

Supplementary data (Tables ST1-ST6 and Cartesian coordinates of all optimized geometries) related to this article can be found at http://dx.doi.org/10.1016/j.jorganchem...

\section{References}

[1] T. P. Fehlner, J.-F. Halet, J.-Y. Saillard, Molecular Clusters. A Bridge to Solid-State Chemistry, Cambridge University Press, New York, 2007.

[2] T. P. Fehlner, Organometallics 19 (2000) 2643-2651.

[3] R. B. King, Inorg. Chem. 43 (2004) 4241-4247.

[4] T. P. Fehlner, Organometallics 19 (2000) 2643-2651.

[5] Y. Nishihara, K. J. Deck, M. Shang, T. P. Fehlner, B. S. Haggerty, A. L. Rheingold, Organometallics 13 (1994) 4510-4522.

[6] X. Lei, M. Shang, T. P. Fehlner, J. Am. Chem. Soc. 120 (1998) 2686-2687.

[7] H. Yan, A. M. Beatty, T. P. Fehlner, Organometallics 21 (2002) 5029-5037.

[8] X. Lei, M. Shang, T. P. Fehlner, Chem. Eur. J. 6 (2000) 2653-2664.

[9] S. Ghosh, B. C. Noll, T. P. Fehlner, Dalton Trans. (2008) 371-378.

[10] K. Geetharani, S. K. Bose, G. Pramanik, T. K. Saha, V. Ramkumar, S. Ghosh, Eur. J. Inorg. Chem. (2009) 1483-1487. 
[11] X. Lei, M. Shang, T. P. Fehlner, J. Am. Chem. Soc. 121 (1999) 1275-1287.

[12] A. S. Weller, M. Shang, T. P. Fehlner, Chem. Commun. (1998) 1787-1788.

[13] B. Le Guennic, H. Jiao, S. Kahlal, J.-Y. Saillard, J.-F. Halet, S. Ghosh, M. Shang, A. M. Beatty, A. L. Rheingold, T. P. Fehlner, J. Am. Chem. Soc. 126 (2004) 3203-3217.

[14] J. Ho, K. J. Deck, Y. Nishihara, M. Shang, T. P. Fehlner, J. Am. Chem. Soc. 117 (1995) 10292-10299.

[15] S. Aldridge, H. Hashimoto, K. Kawamura, M. Shang, T. P. Fehlner, Inorg. Chem. 37 (1998) 928-940.

[16] H. J. Bullick, P. D. Grebenik, M.L. H. Green, A. K. Hughes, J. B. Leach, P. C. McGowan, J. Chem. Soc. Dalton Trans. (1995) 67-75.

[17] S. Aldridge, M. Shang, T. P. Fehlner, J. Am. Chem. Soc. 120 (1998) 2586-2598.

[18] S. Sahoo, R. S. Dhayal, B. Varghese, S. Ghosh, Organometallics 28 (2009) 1586-1589.

[19] A. S. Weller, M. Shang, T. P. Fehlner, Organometallics 18 (1999) 53-64.

[20] S. K. Bose, S. Ghosh, B. C. Noll, J.-F. Halet, J.-Y. Saillard, A. Vega, Organometallics 26 (2007) 5377-5385.

[21] C. Ting, L. Messerle, J. Am. Chem. Soc. 111 (1989) 3449-3450.

[22] S. Aldridge, H. Hashimoto, M. Shang, T. P. Fehlner, J. Chem. Soc. Chem. Commun. (1998) 207-208.

[23] S. K. Bose, K. Geetharani, B. Varghese, S. M. Mobin, S. Ghosh, Chem. Eur. J. 14 (2008) 9058-9064.

[24] T. Onak, J. Tseng, M. Diaz, D. Tran, J. Arias, S. Herrera, D. Brown, Inorg. Chem. 32 (1993) 487-489.

[25] G. Schreckenbach, T. Ziegler, Theor. Chem. Acc. 99 (1998) 71-82. 
[26] M. Bühl, D. Hnyk, J. Macháček, Chem. Eur. J. 11 (2005) 4109-4120.

[27] M. Bühl, J. Holub, D. Hnyk, J. Macháček, Organometallics 25 (2006) 2173-2181.

[28] M. Bühl, D. Hnyk, J. Macháček, Inorg. Chem. 46 (2007) 1771-1777.

[29] K. Geetharani, B. S. Krishnamoorthy, S. Kahlal, S. M. Mobin, J.-F. Halet, S. Ghosh, Inorg. Chem. 51 (2012) 10176 - 10184.

[30] B. S. Krishnamoorthy, A. Thakur, K. Chakrahari, S. Bose, P. Hamon, T. Roisnel, S. Kahlal, S. Ghosh, J.-F. Halet, Inorg. Chem. 51 (2012) 10375 - 10383.

[31] B. S. Krishnamoorthy, S. Kahlal, S. Ghosh, J.-F Halet, Theor. Chem. Acc. 132 (2013) 1356-1366.

[32] B. S. Krishnamoorthy, S. Kahlal, B. Le Guennic, J.-Y. Saillard, S. Ghosh, J.-F. Halet Sol. State Sci. 14 (2012) 1617-1623.

[33] S. K. Bose, S. M. Mobin, S. Ghosh, J. Organomet. Chem. 696 (2011) 3121-3126.

[34] A. S. Weller, T. P. Fehlner, Organometallics 18 (1999) 447-450.

[35] Y. Nishihara, K. J. Deck, M. Shang, T. P. Fehlner, V. A. Haggerty, L. Rheingold, Organometallics 13 (1994) 4510-4522.

[36] ADF2010.02, SCM, Theoretical Chemistry, Vrije Universiteit, Amsterdam, The Netherlands, http://www.scm.com.

[37] G.teVelde, F. M. Bickelhaupt, E. J. Baerends, S.J. A.van Gisbergen, C. Fonseca Guerra, J. G. Snijders, T. Ziegler, J. Comput. Chem. 22 (2001) 931-967.

[38] S. H. Vosko, L. Wilk, M. Nusair, Can. J. Phys., 58 (1980) 1200-1211.

[39] A. D. Becke, J. Chem. Phys. 84 (1986) 4524-4529.

[40] A. D. Becke, Phys. Rev. A 38 (1986) 3098-3100. 
[41] J. P. Perdew, Phys. Rev. B 33 (1986) 8822-8824.

[42] L. Versluis, T. Ziegler, J. Chem. Phys. 88 (1988) 322-329.

[43] E. van Lenthe, E. J. Baerends, J. G. Snijders, J. Chem. Phys. 99 (1993) 4597-4610.

[44] E. van Lenthe, E. F. Baerends, J. G. Snijders, J. Chem. Phys. 101 (1994) 9783-9792.

[45] E. van Lenthe, R. van Leeuwen, E. J. Baerends, J. G. Snijders, Int. J. Quantum. Chem. 57 (1996) 281-293.

[46] A. D. Becke, Phys. Rev. A, 38 (1988) 3098-3100.

[47] C. Lee, W. Yang, R. G. Parr, Phys. Rev. B 37 (1988) 785-789.

[48] A. D. Becke, J. Chem. Phys., 98 (1993) 5648-5652.

[49] F. London, J. Phys. Radium 27 (1937) 397-409.

[50] R. Ditchfield, Mol. Phys. 27 (1974) 789-807.

[51] K. Wolinski, J. F. Hinton, P. Pulay, J. Am. Chem. Soc. 112 (1990) 8251-8260.

[52] K. Friedrich, G. Seifert, G. Grossmann, Z. Phys. D, 17 (1990) 45-46.

[53] G Schreckenbach, T. Ziegler, J. Phys. Chem. 99 (1995) 606-611.

[54] G. Schreckenbach, T. Ziegler, Int. J. Quantum. Chem. 61 (1997) 899-918.

[55] G. Schreckenbach, T. Ziegler, Int. J. Quantum. Chem. 60 (1996) 753-766.

[56] S. K. Wolff, T. Ziegler, J. Chem. Phys. 109 (1998) 895-905.

[57] S. K. Wolff, T. Ziegler, E. van Lenthe, E. J. Baerends, J. Chem. Phys. 110 (1999) 76897698.

[58] T. P. Fehlner, Collect. Czech. Chem. Commun. 64 (1999) 767-782. 
[59] S. K. Bose, K. Geetharani, V. Ramkumar, S. M. Mobin, S. Ghosh, Chem. Eur. J. 15 (2009) 13483-13490.

[60] J. Emsley, The Elements, Oxford University Press, Oxford, 3rd Ed., 1998.

[61] K. J. Deck, Y. Nishihara, M. Shang, T. P. Fehlner, J. Am. Chem. Soc. 116 (1994) 84088409.

[62] S. K. Bose, S. Ghosh, B. C. Noll, J.-F. Halet, J.-Y. Saillard, A. Vega, Organometallics 26 (2007) 5377-5385.

[63] S. Ghosh, M. Shang, T. P. Fehlner, J. Organomet. Chem., 614-615 (2000) 92-98.

[64] M. David Curtis, W. M. Butler, J. Organomet. Chem. 155 (1978) 131-145.

[65] K. Wade, J. Chem. Soc. D. (1971) 792-793.

[66] K. Wade, Adv. Inorg. Chem. Radiochem. 18 (1976) 1-66.

[67] K. Wade, in Transition Metal Clusters, B. F. G. Johnhson (ed.), Wiley, Chichester, 1980, pp. 193-264.

[68] D.M.P. Mingos, Nature Phys. Sci. 236 (1972) 99-102.

[69] D. M. P. Mingos, Acc. Chem. Res. 17 (1984) 311-319.

[70] D. M. P. Mingos, D. J. Wales, Introduction to Cluster Chemistry, Prentice-Hall, Englewood Cliffs, 1990.

[71] R. B. King, Inorg. Chem. 45 (2006) 8211-8216.

[72] R. B. King, S. Ghosh, Theor. Chem. Acc. 131 (2012) 1087. 
[73] J.-F. Halet, J.-Y. Saillard, in: J. Reedijk, K. Poeppelmeier (eds.), Comprehensive Inorganic Chemistry II, Elsevier, Oxford, 2013 vol. 9: Theory and Methods (S. Alvarez, volume ed.), p. 869-885.

[74] D. M. Hoffman, R. Hoffmann, C. R. Fisel, J. Am. Chem. Soc. 104 (1982) 3858-3875.

[75] J. Autschbach, T. Ziegler, in: Encyclopedia of Nuclear Magnetic Resonance, Vol. 9, Advances in NMR, John Wiley and Sons, Chichester, 2002, pp. 306-323. 


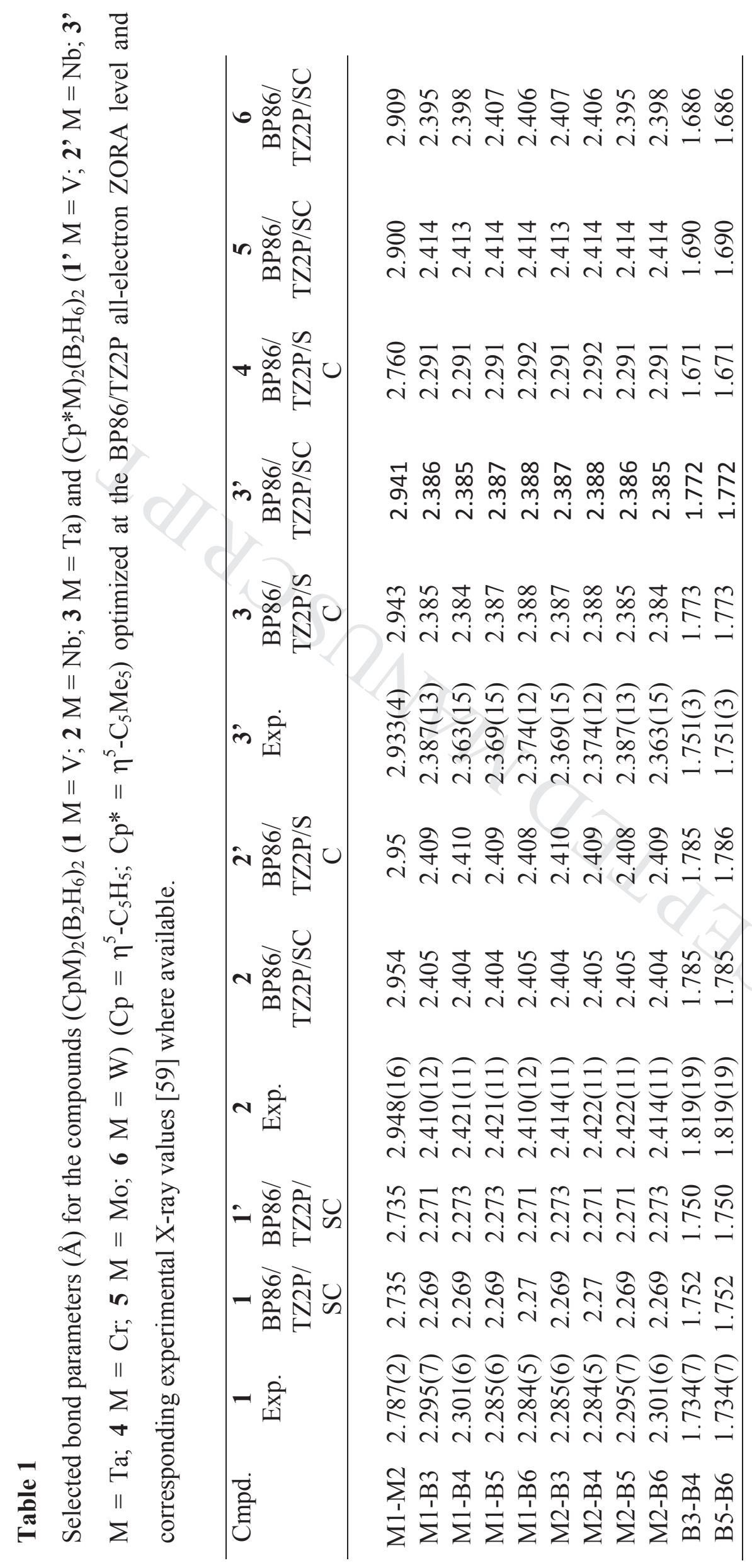




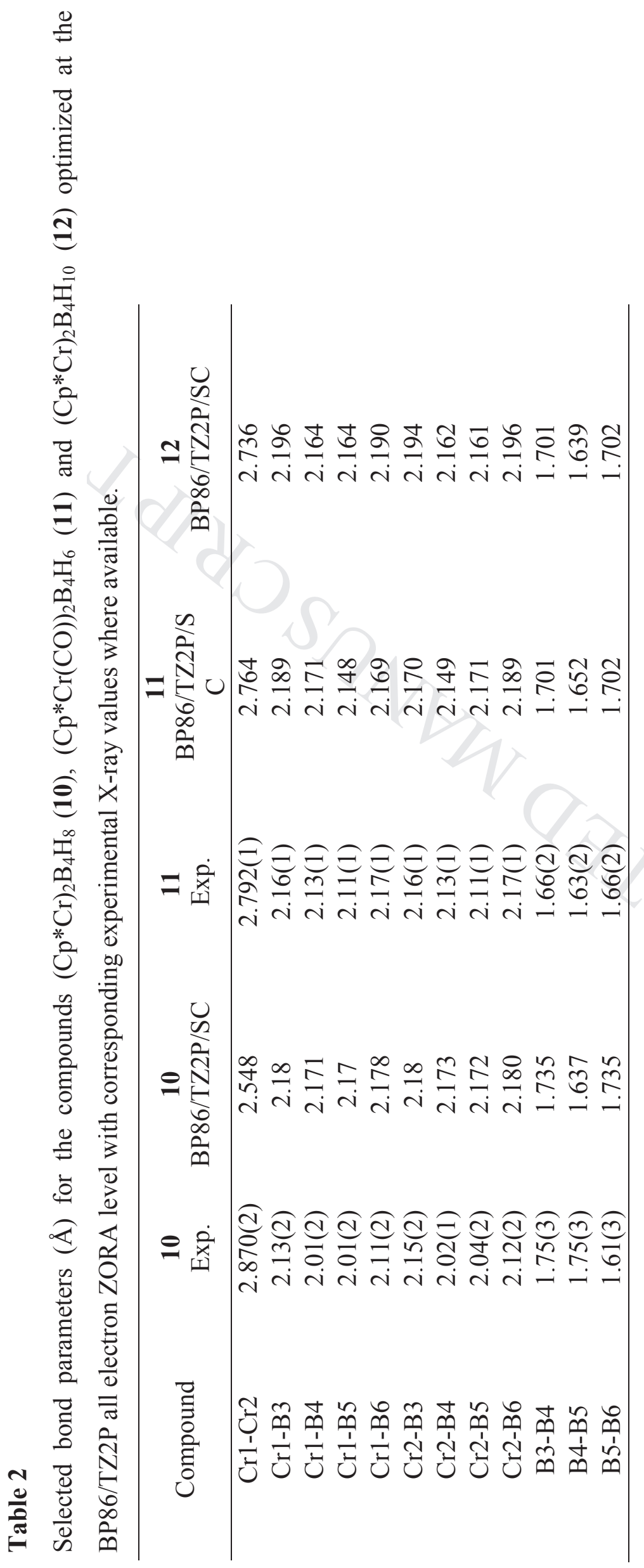




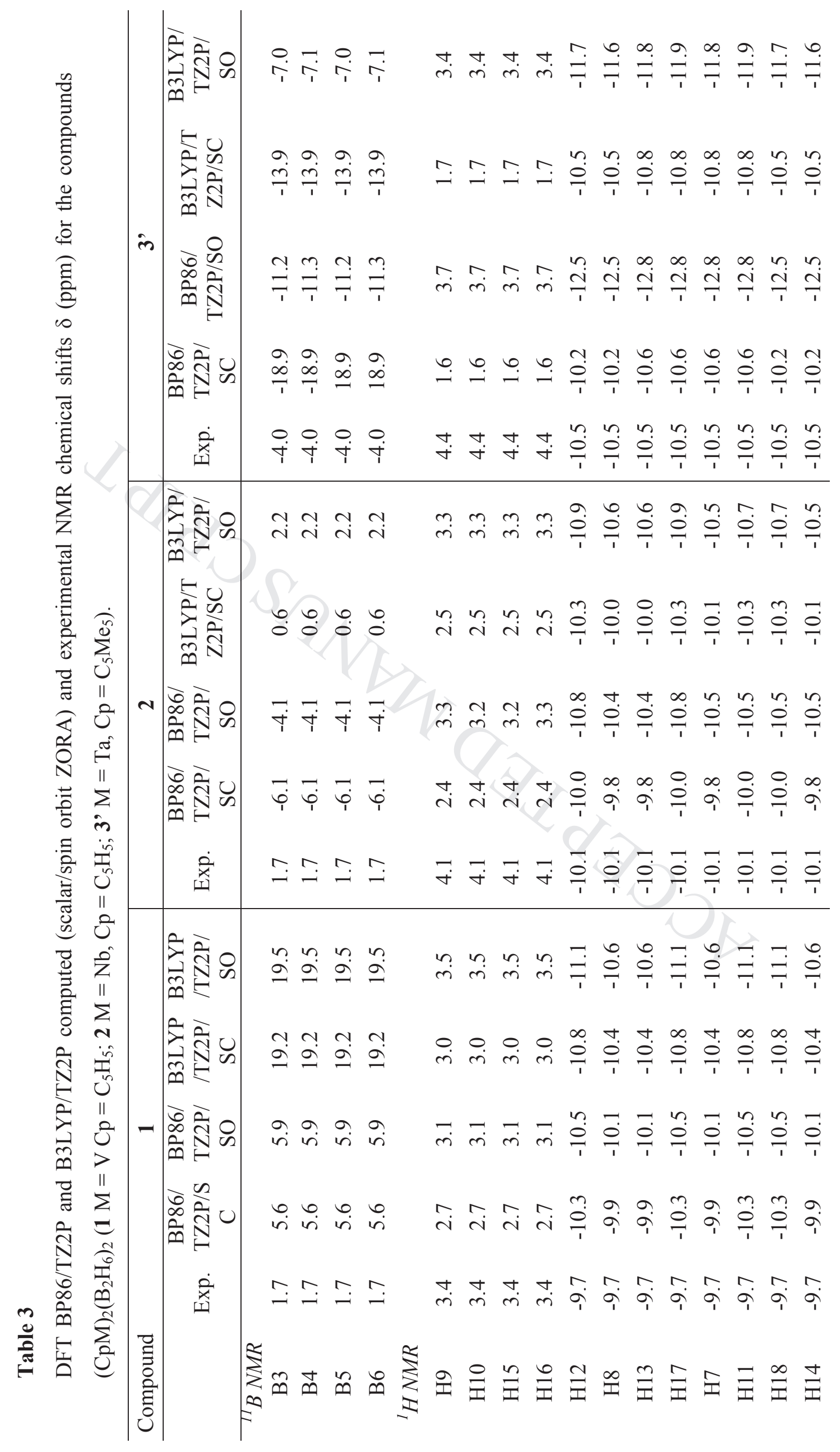


$\vec{i} \quad \stackrel{n}{0}=$

$\stackrel{0}{i} \quad \stackrel{0}{0}$

ㄱ. $\stackrel{\stackrel{m}{g}}{=} \stackrel{\infty}{0}$

$\stackrel{+}{\stackrel{\varpi}{g}} \stackrel{?}{=}$

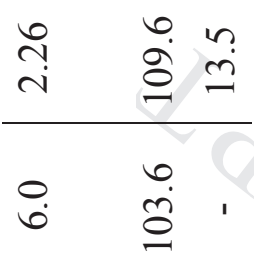

iे $\stackrel{0}{\text { ḋ }}$

․

용.

in $\frac{1}{a}$.

ir $\vec{a}$

in a

ìn

in $\stackrel{0}{a}$.

하 $\stackrel{\infty}{a}$.

$\sum_{0}^{\pi} \sum_{0}^{\infty} U^{0} U^{0}$ 


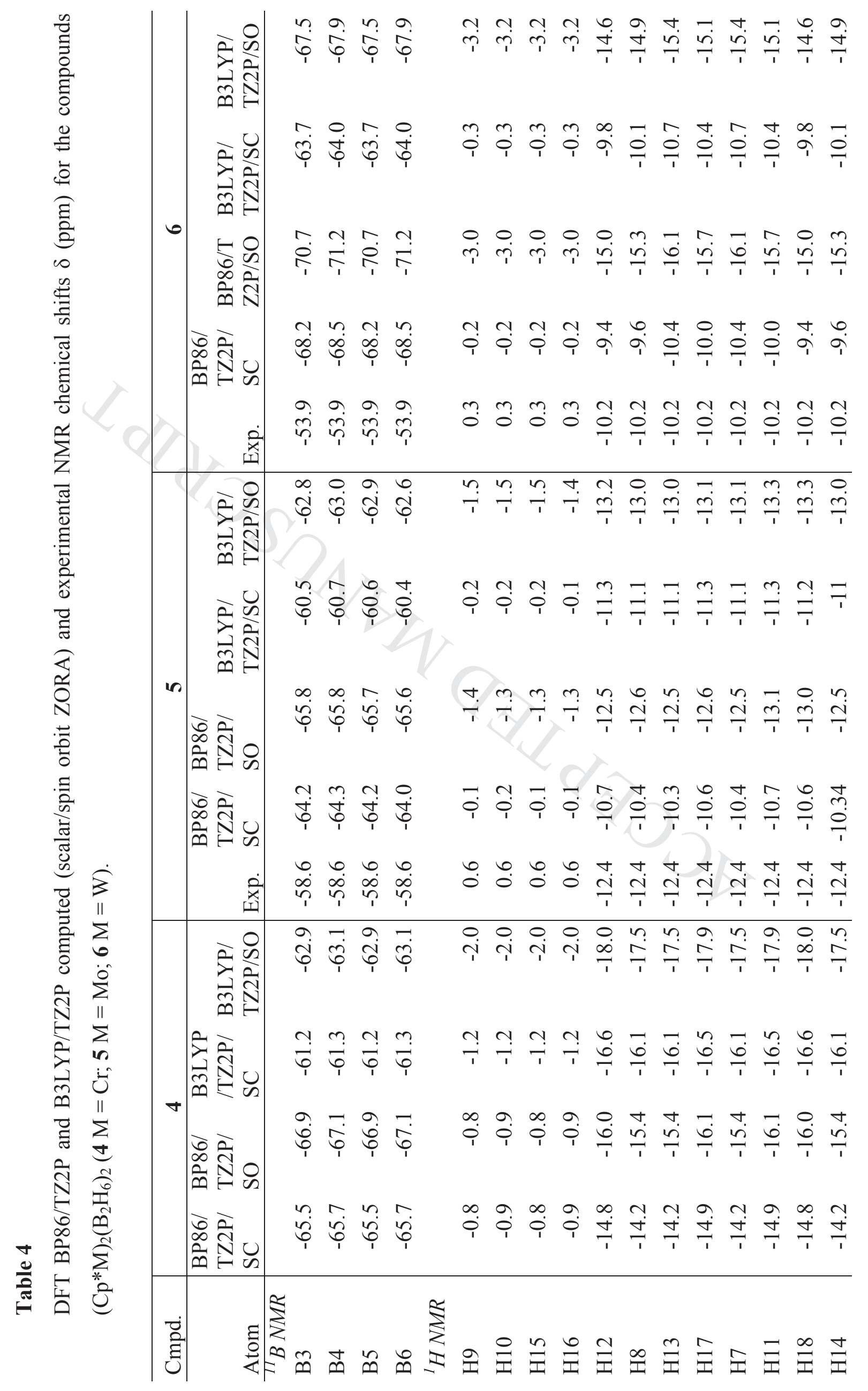




$$
\begin{aligned}
& \text { ․ } \quad \begin{array}{lll}
0 & 0 \\
0 & 0
\end{array} \\
& \text { ․ }
\end{aligned}
$$

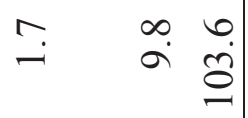

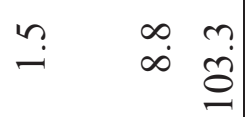

$$
\begin{aligned}
& \text { I. }
\end{aligned}
$$

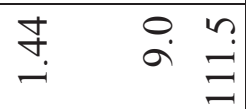

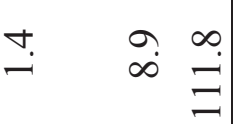

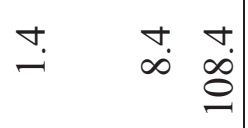

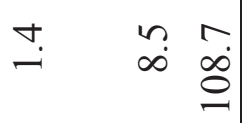

$$
\begin{aligned}
& \overrightarrow{0} \quad \overrightarrow{0}
\end{aligned}
$$

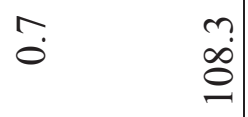

$$
\begin{aligned}
& \hat{0} \\
& \stackrel{0}{0}
\end{aligned}
$$

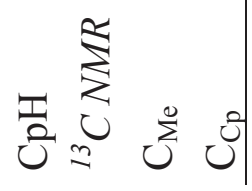




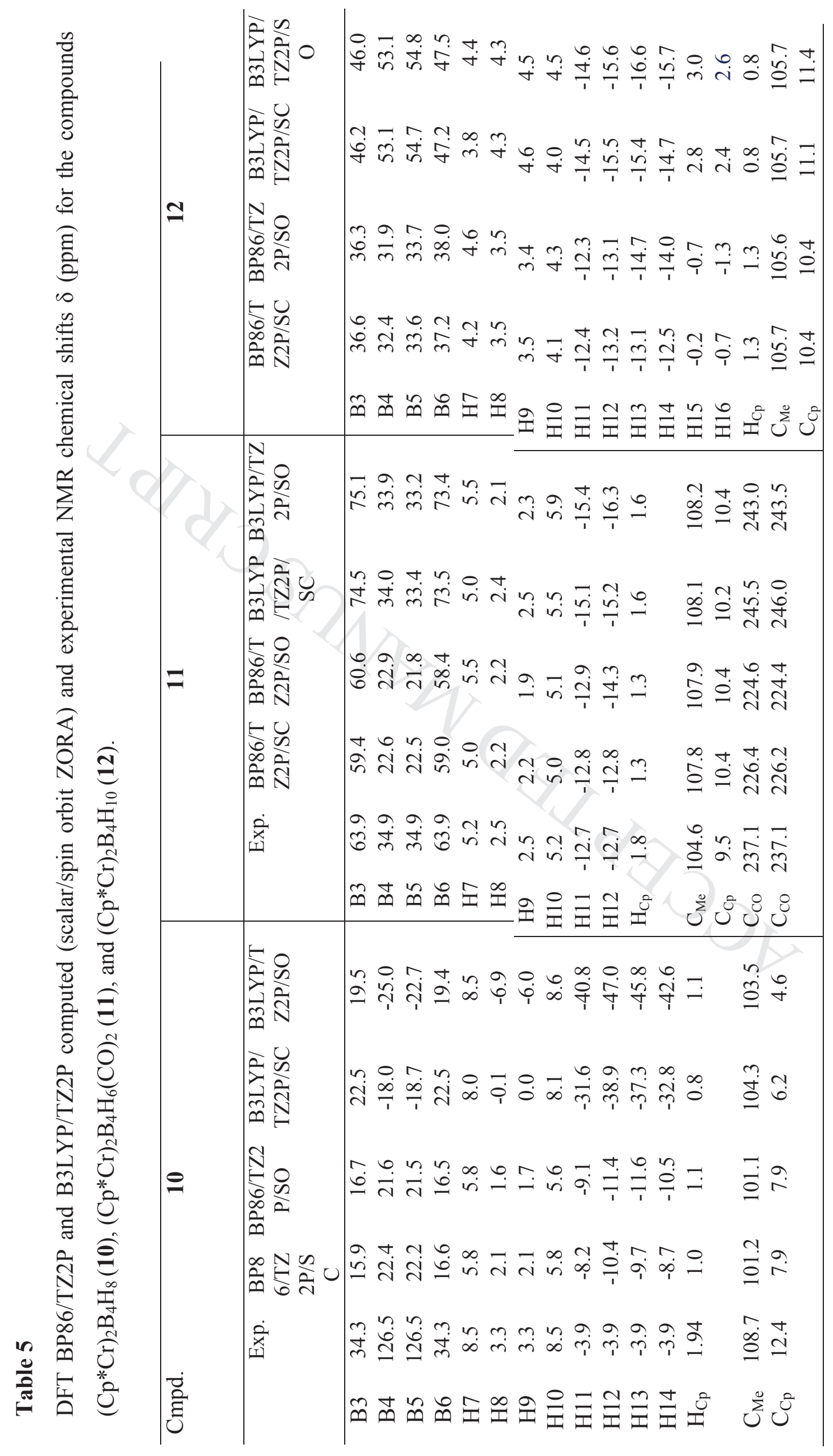




\section{Structural, electronic and magnetic properties of some early vs late transition dimetallaborane clusters - A theoretical investigation}

Kandasamy Bharathi ${ }^{\mathrm{a}}$, Lalshab Beerma ${ }^{\mathrm{a}}$, Chinnasamy Santhi ${ }^{\mathrm{a}}$, Bellie Sundaram Krishnamoorthy ${ }^{\mathrm{a}, \mathrm{b}, *}$ and Jean-François Halet ${ }^{\mathrm{b}, *}$

${ }^{a}$ Department of Chemistry, Vivekanandha College of Arts and Sciences for Women (Autonomous), Elayampalayam, Tiruchengode, 600 036, India

${ }^{b}$ Institut des Sciences Chimiques de Rennes, UMR 6226 CNRS-Université de Rennes 1, Avenue du Général Leclerc, 35042 Rennes Cédex, France

\section{Highlights}

The strength of DFT methods in analyzing the electronic and magnetic properties of a series of dimetallaboranes is demonstrated $\square{ }^{11} \mathrm{~B}$ chemical shifts are well reproduced at the DFTGIAO BP86/TZ2P level for first-row transition metal compounds B3LYP/TZ2P/SO level is necessary for second- and third-row transition metal compounds 


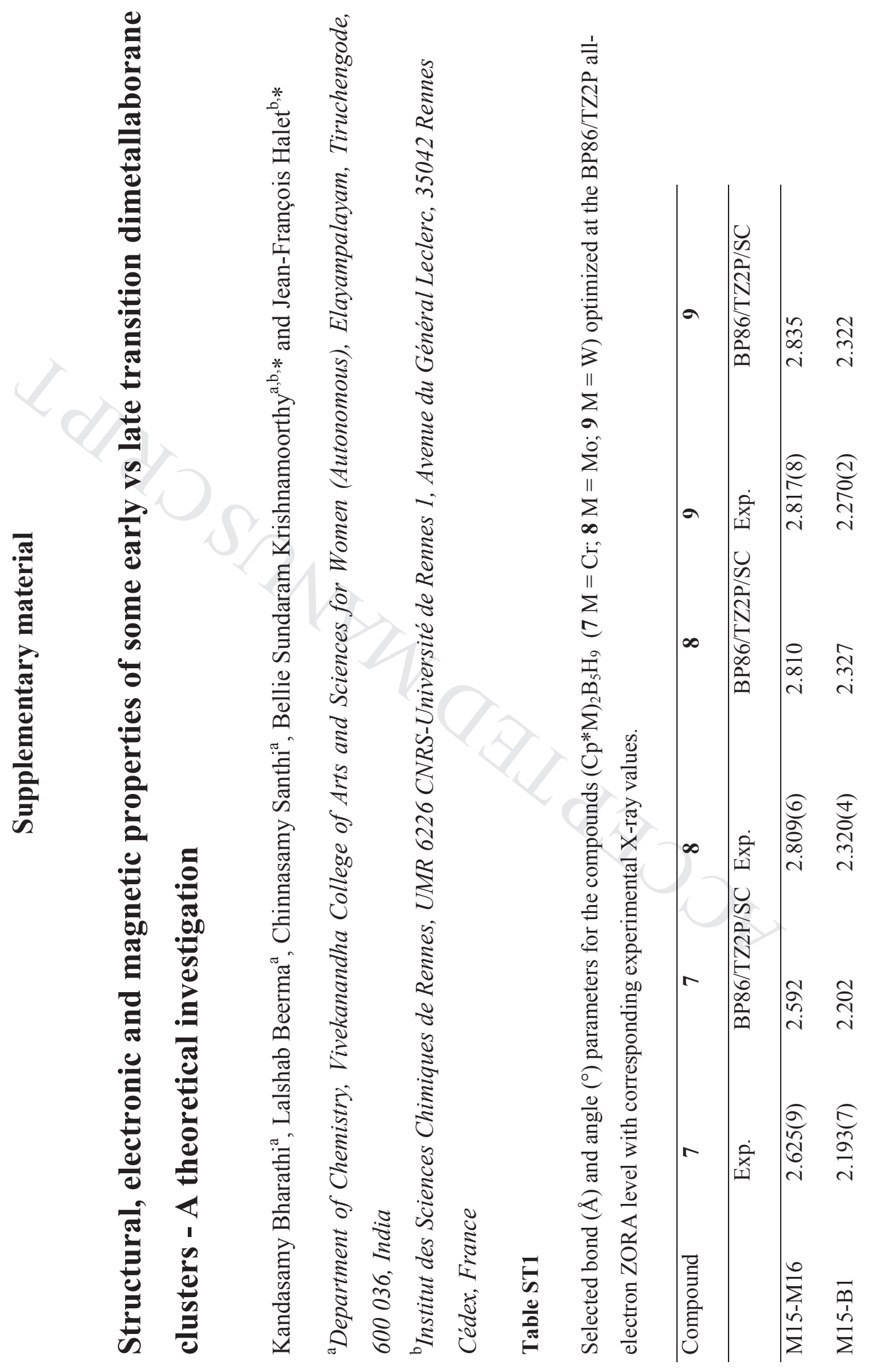




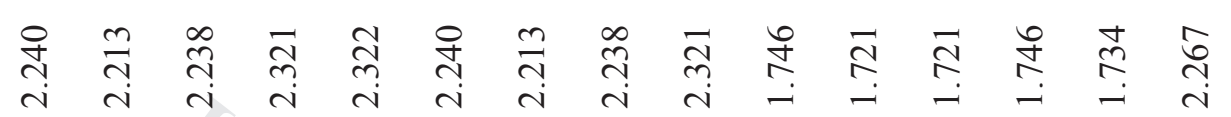

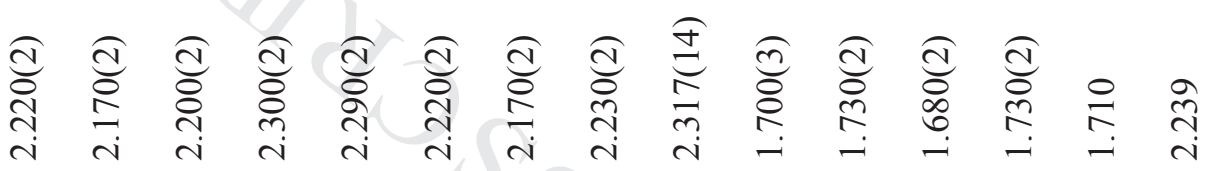

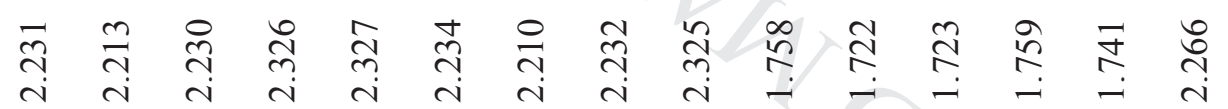

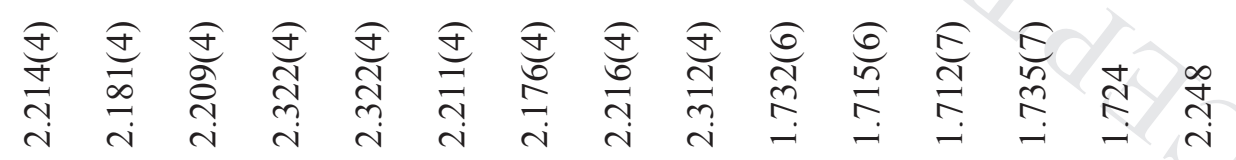

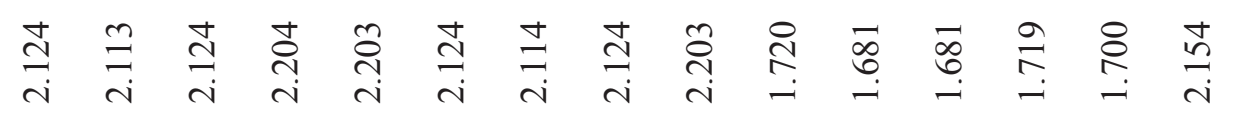

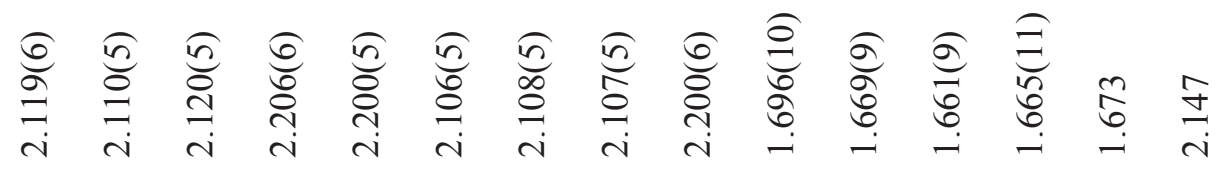

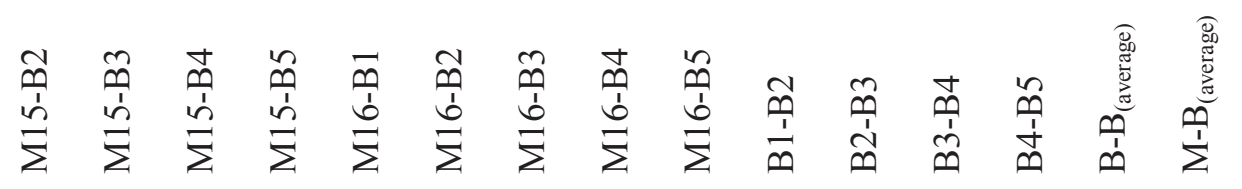




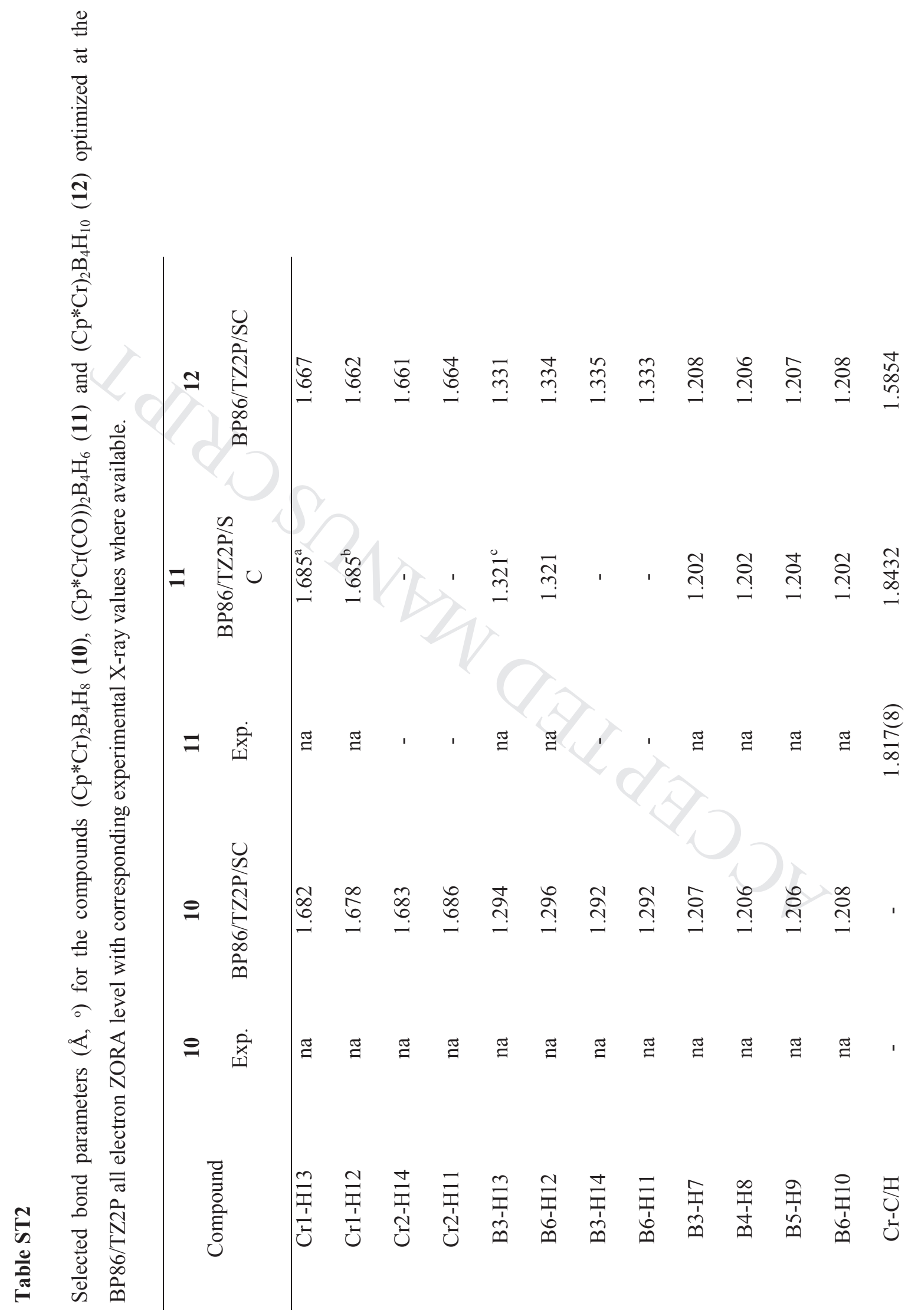




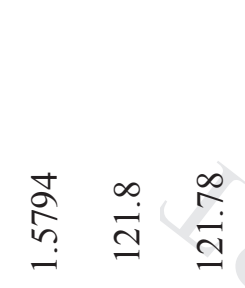

疍

$\cong \stackrel{\infty}{\stackrel{\infty}{3}}$.

$-$

$\cong \bar{\Xi}$

$\Xi \varepsilon$

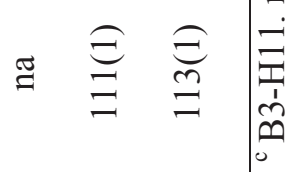

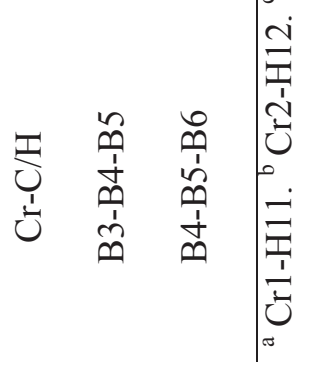

六

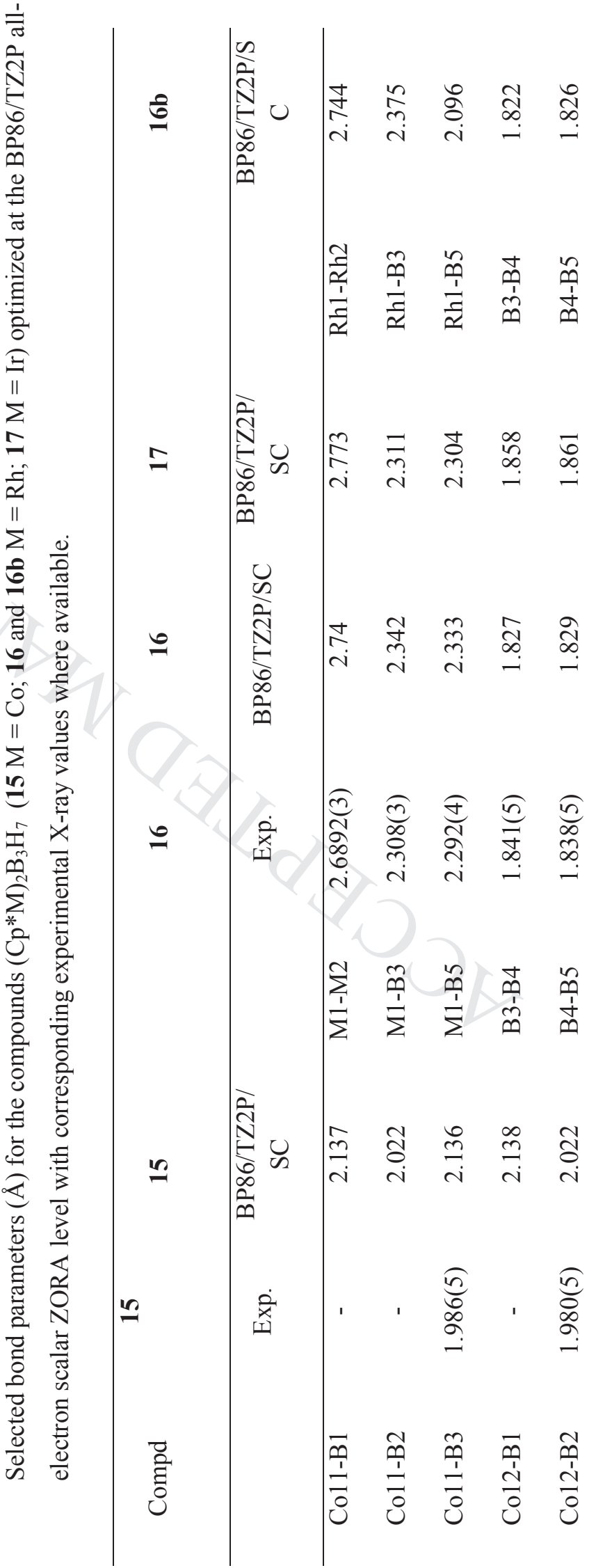




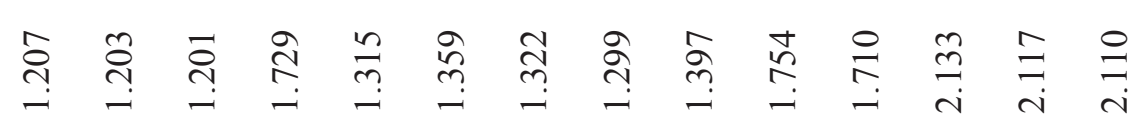

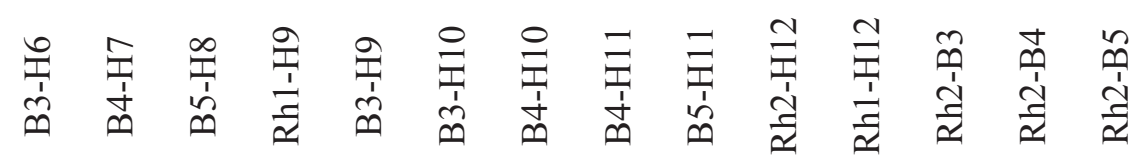

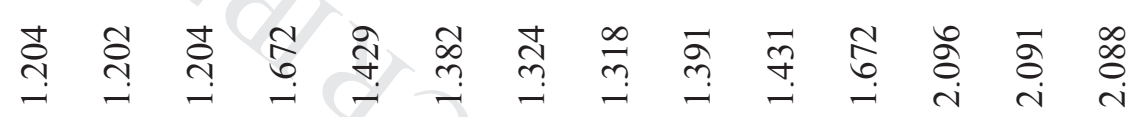

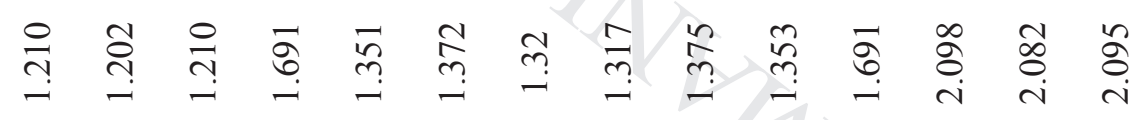

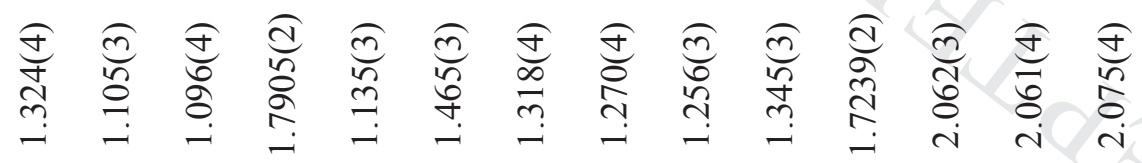

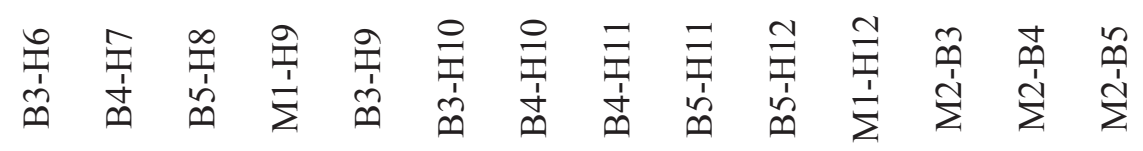

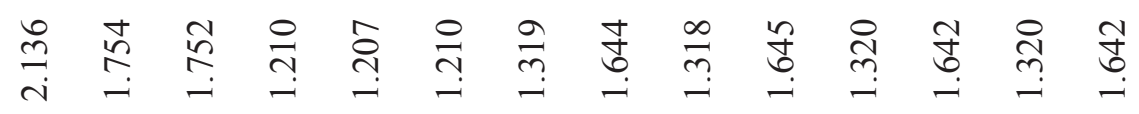

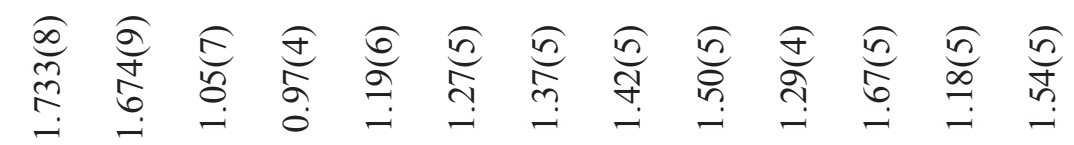

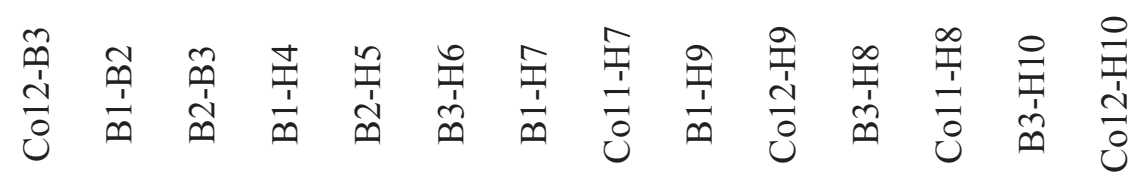




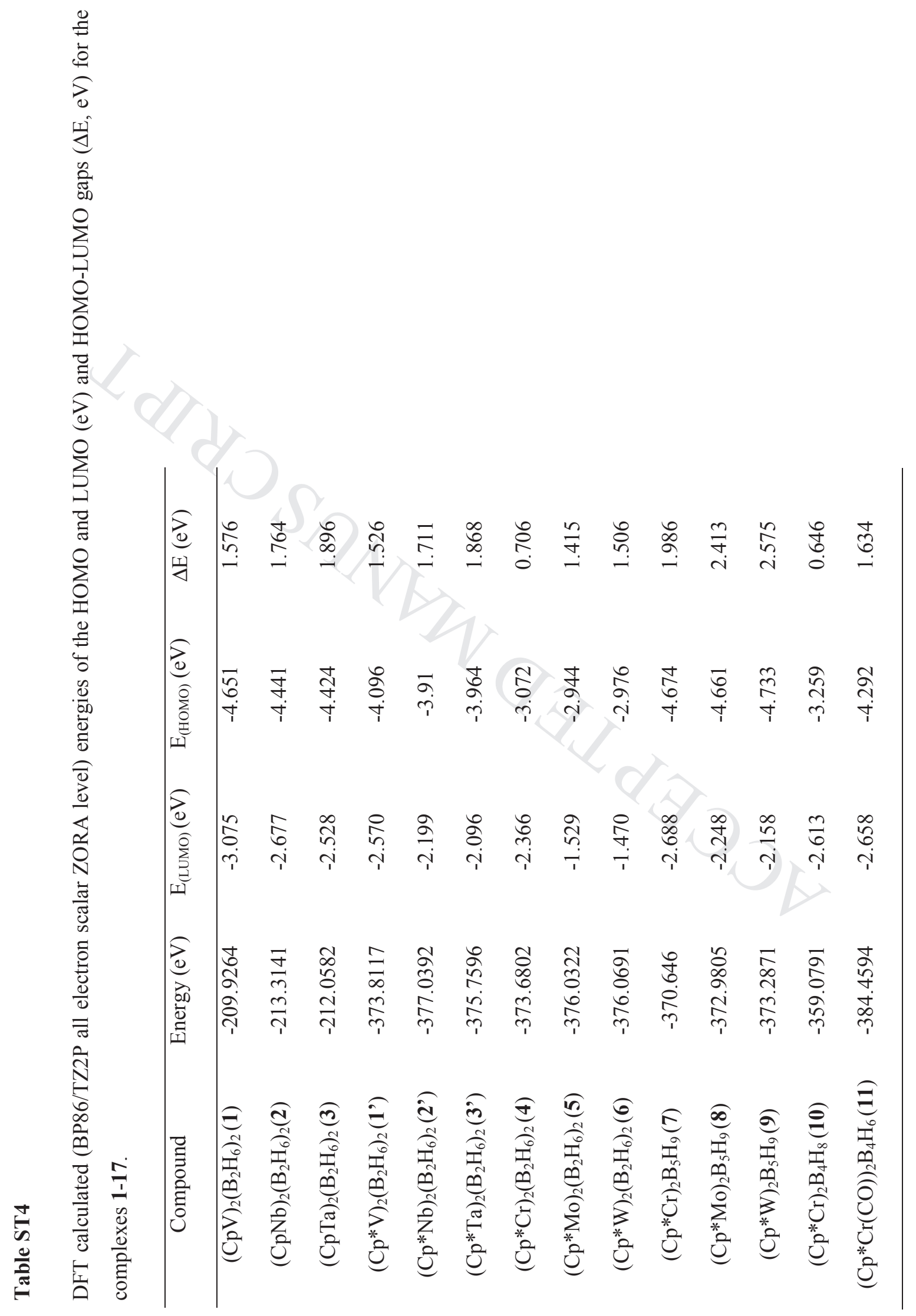




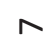

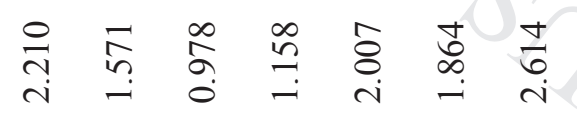

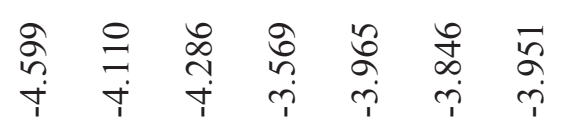

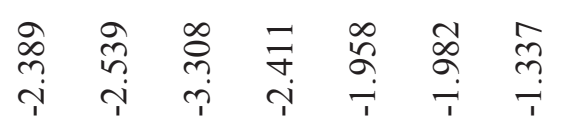

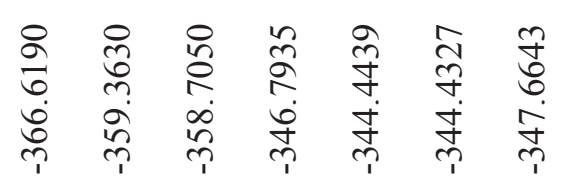

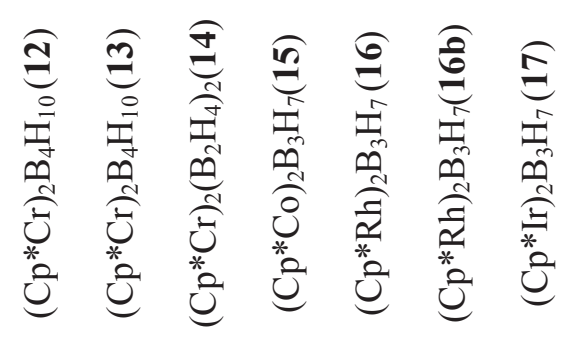




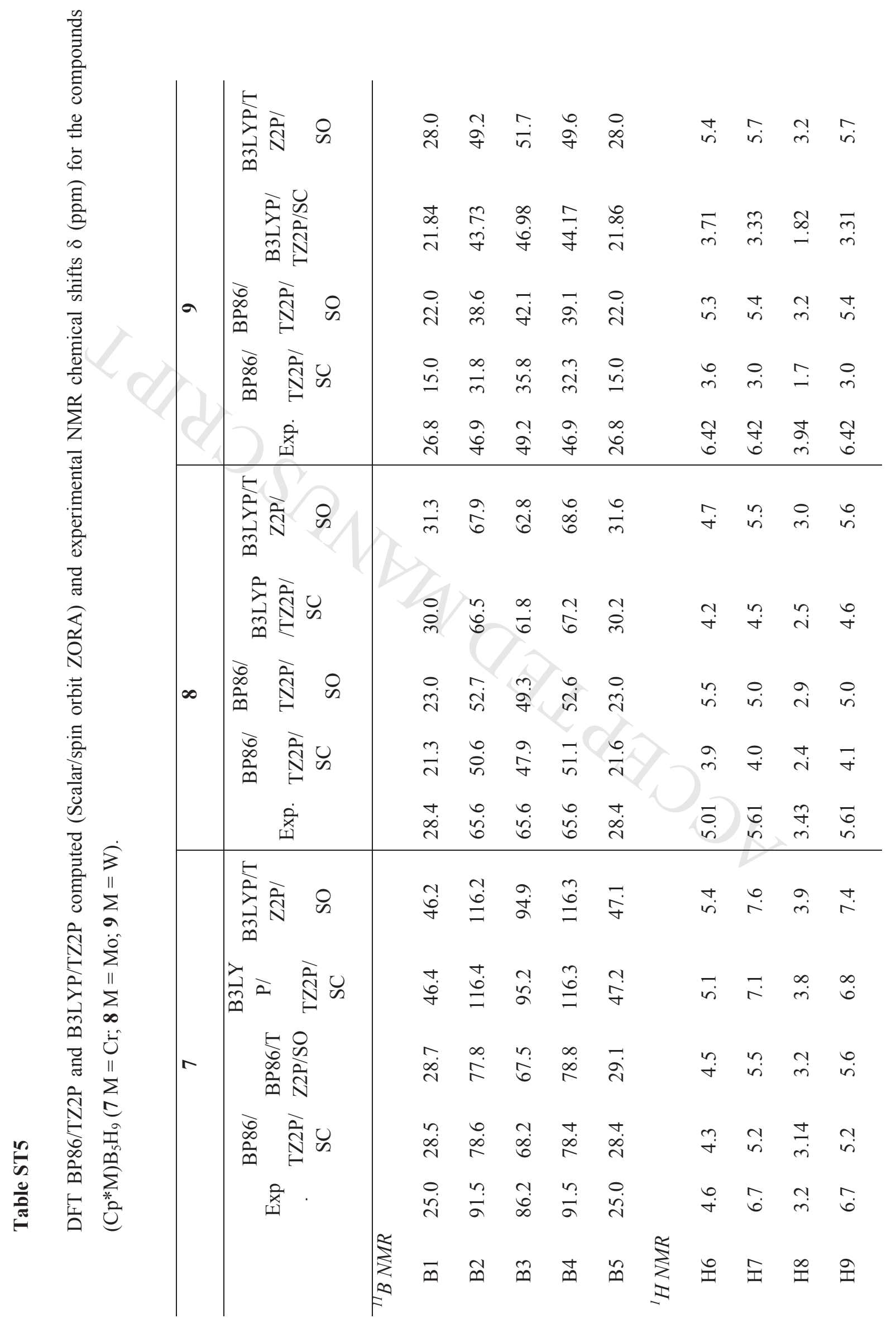




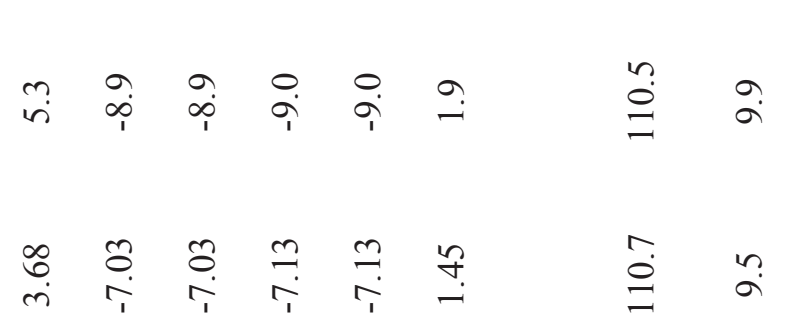

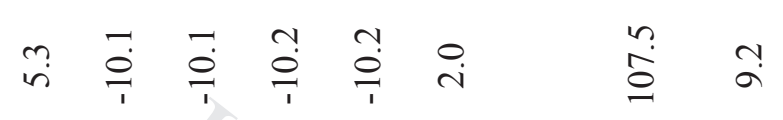

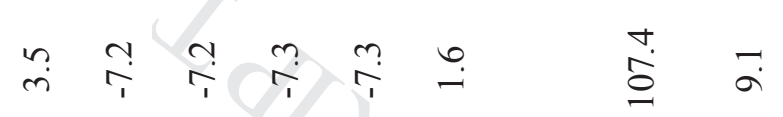

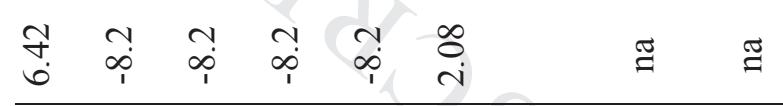

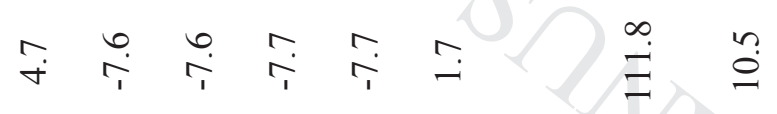

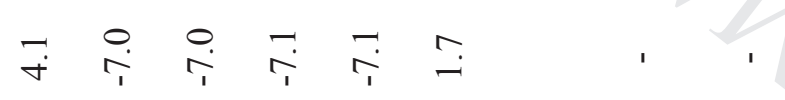

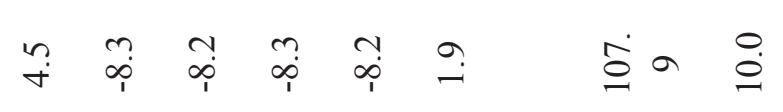

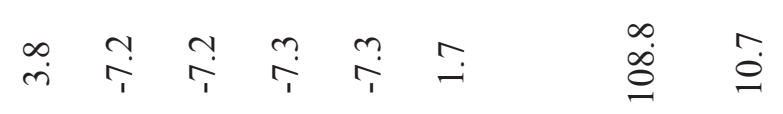

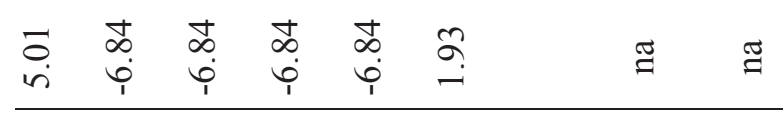

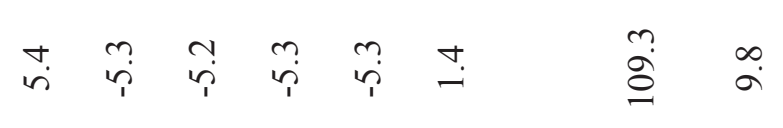

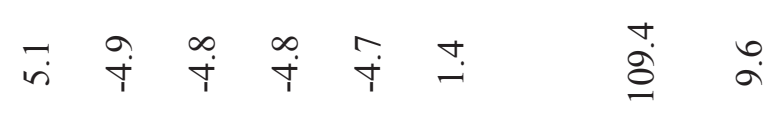

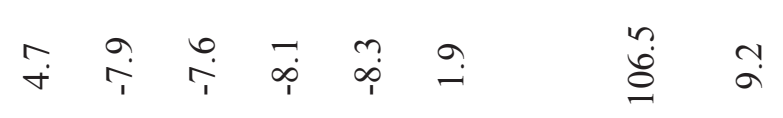

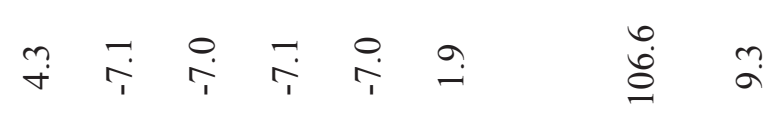

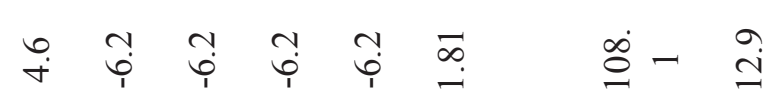

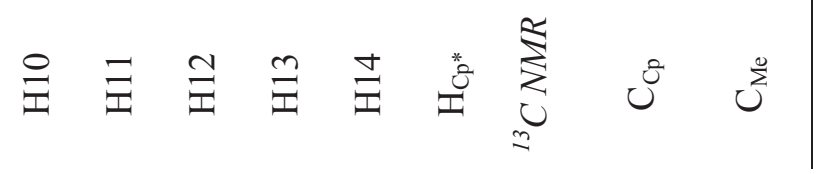




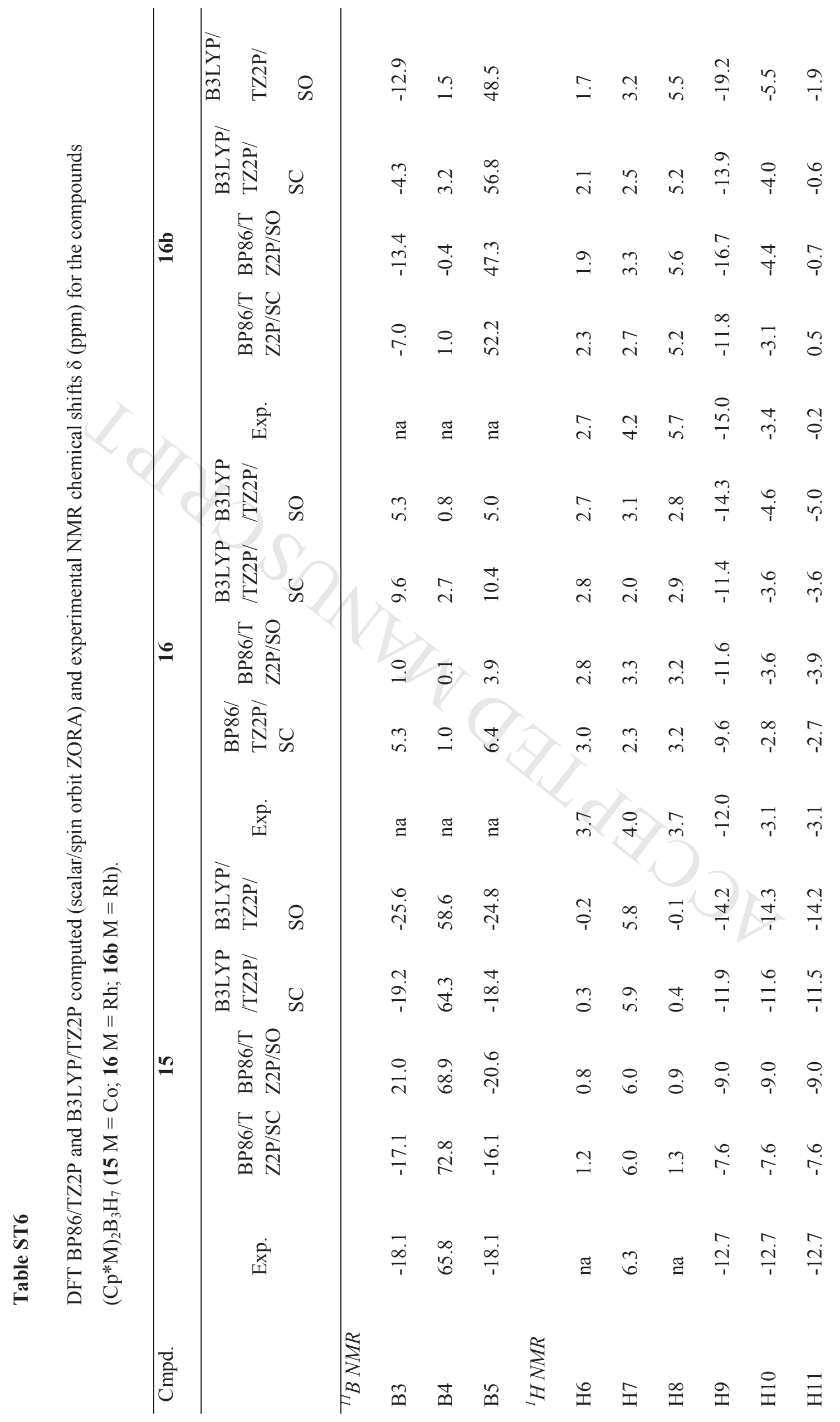




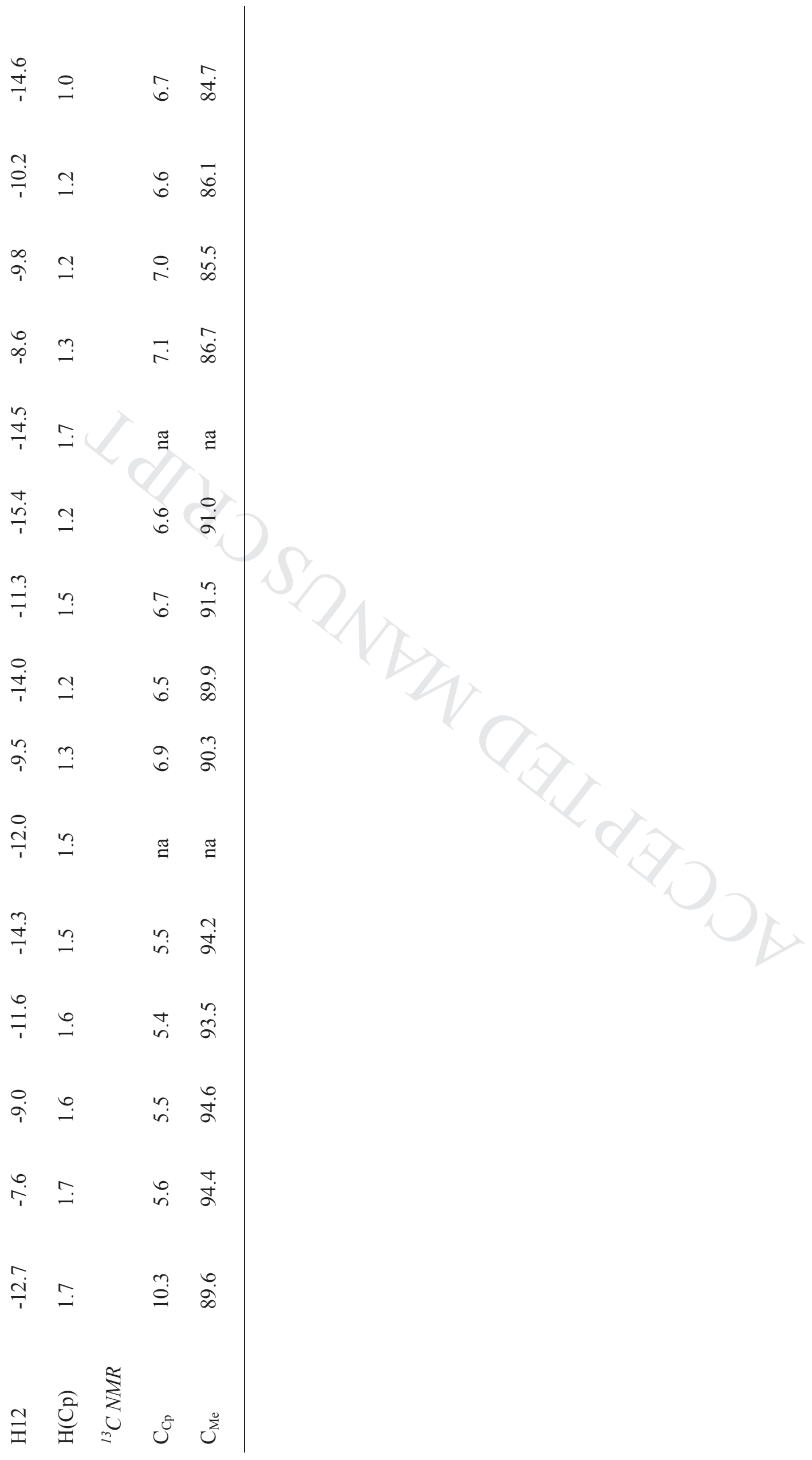

$\exists$ 


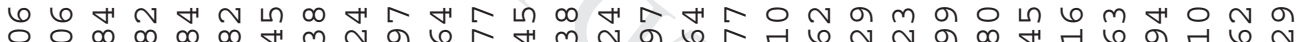

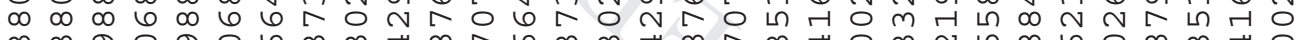

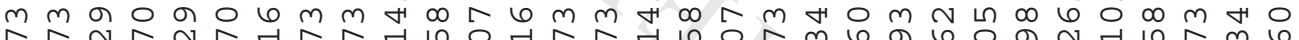
बิ人

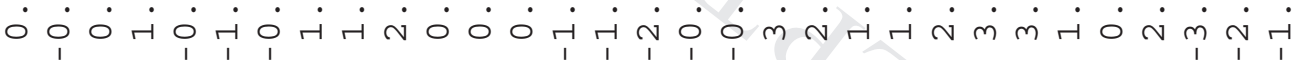

の न त 0 - 0 r H

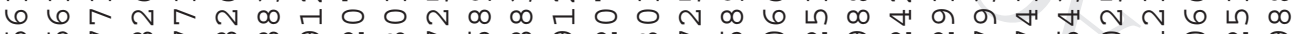

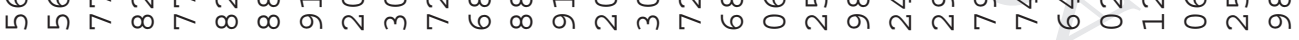

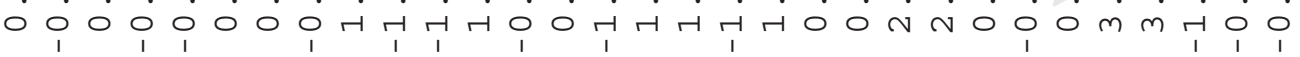

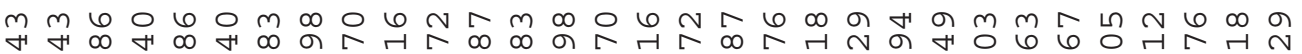
A

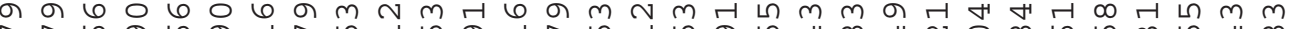
^尺

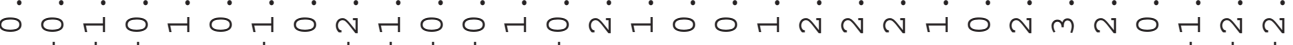

$P \triangleright \sqcap \sqcap \sqcap \sqcap$ 出出出出出出出出出出出出UUUUU出出出出出UUU - $N$ m 


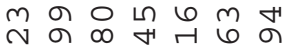
$m \rightarrow \operatorname{N} \infty \sim$ $m \sim 10 \infty 60$ ब 60 ब $N$ त เ स व 0 क मी

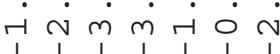

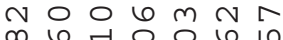

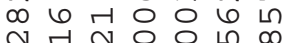
$N \pi N 000$ 걱 ฟ

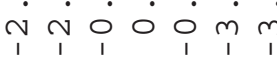

मั भ बे बून भ मे मे $\forall r 0060$

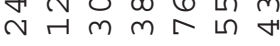

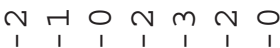

U出出出出出 $\stackrel{m}{m} \dot{m} \stackrel{n}{m} \hat{m} \stackrel{\infty}{m}$

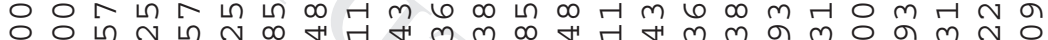

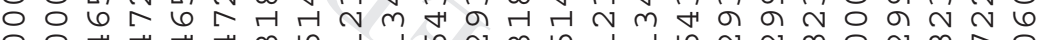

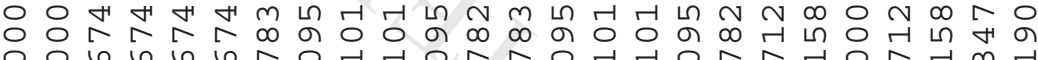

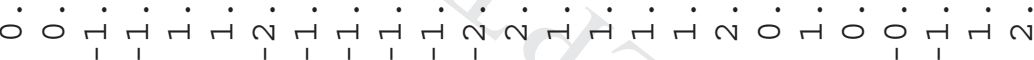

"の유요 6ก の

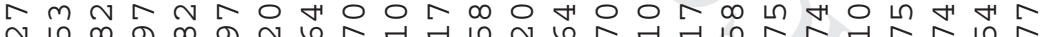
ஸ 긍ำ

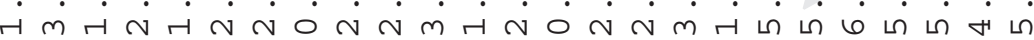

の

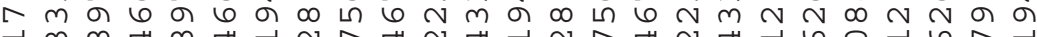
긋

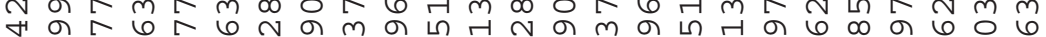
ナூ

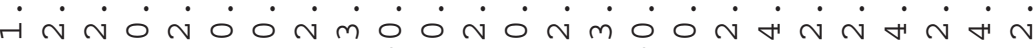

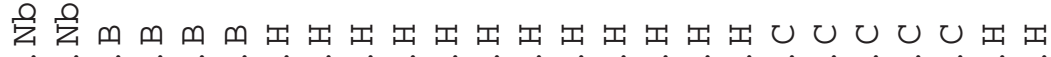

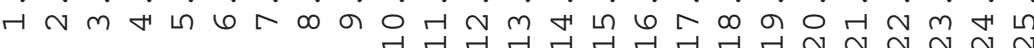


웡유

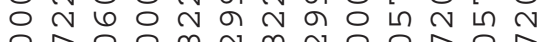
$0 \pi \circ 0 \infty N \infty N$ म ब

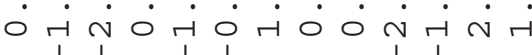

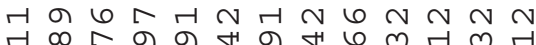
댁

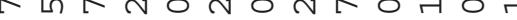
$m$ เ $\infty$ เ $m$ ब $m$ बता

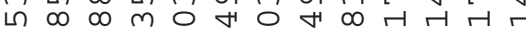

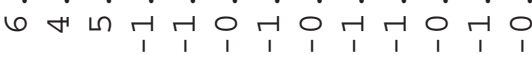

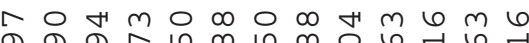

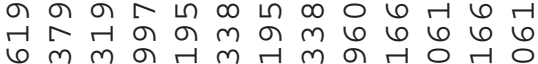

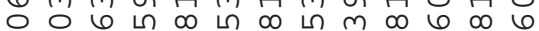
त ब

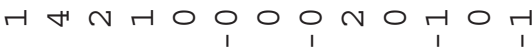

出出出UUUU兀出出出出出

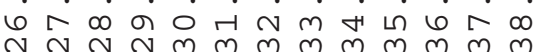

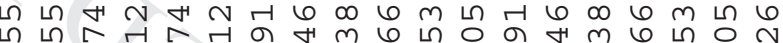

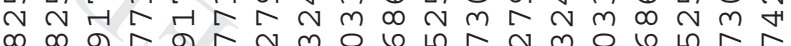

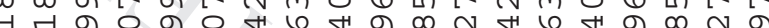

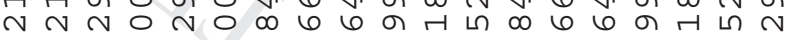

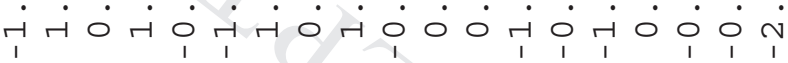

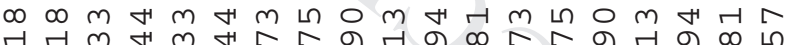

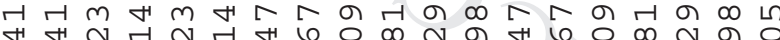

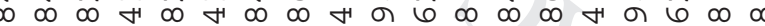
$0 \quad \infty$ เ $\infty$ L เ

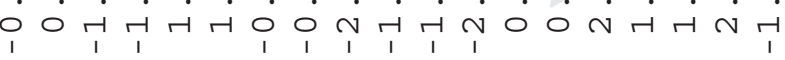

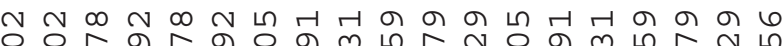

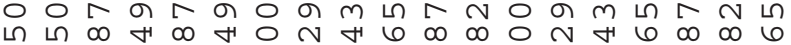
$\Omega$

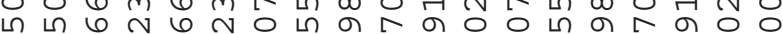
மூ

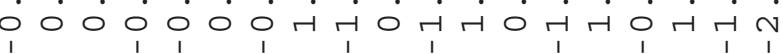

E

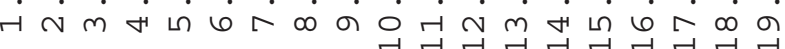




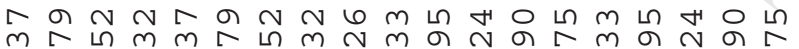

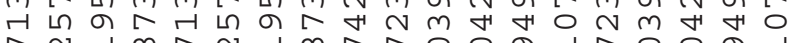

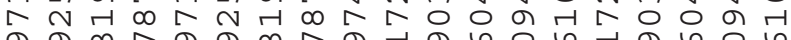
の

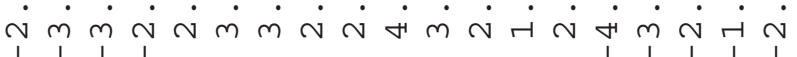

○ N

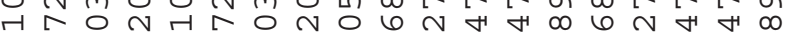
$m 6 m 6 m 6 m 6 \infty N+\infty 0 m N+\infty \circ m$

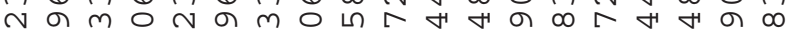

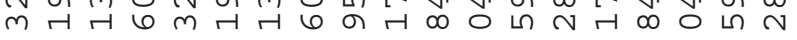

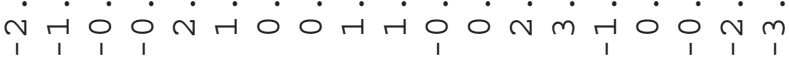

ด人

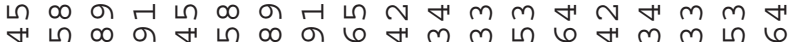
अ

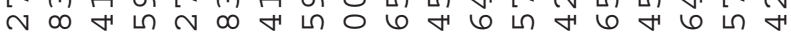
○ क म r

$\circlearrowright U U U U \circlearrowright U U U$ 出出出出出出出出出出

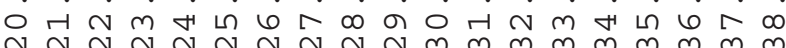

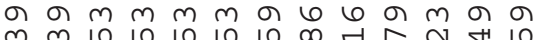
$m$ b

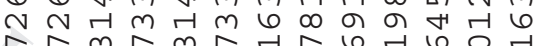
बิ

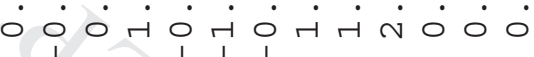

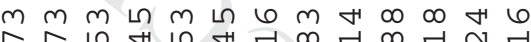

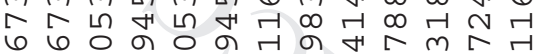

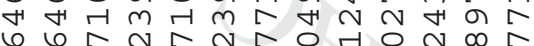
เที่ำ

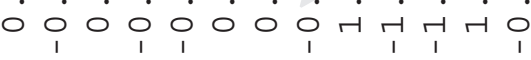

댄대

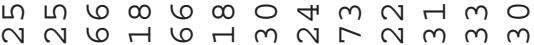

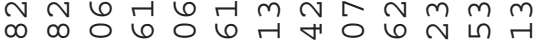
- 1 a

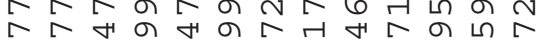

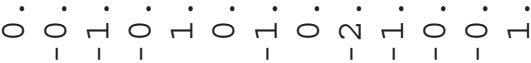

アメのッロ出出出出出出出

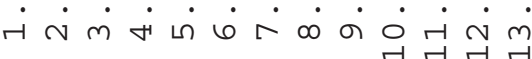




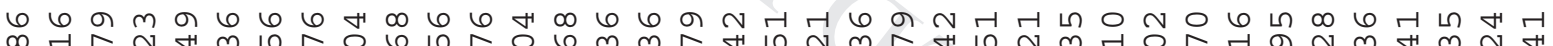
$r-r \infty \omega n$ L $\infty$ ब $ब$ म

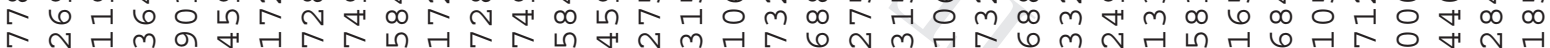

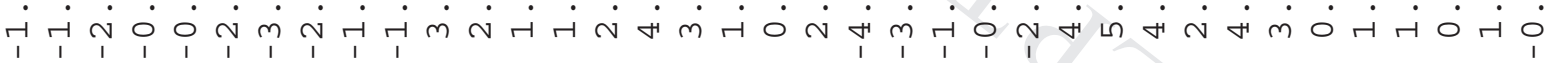

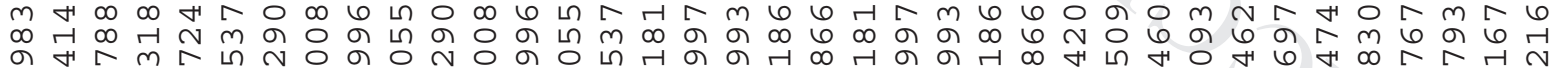

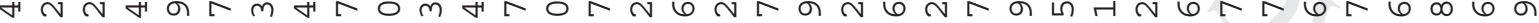

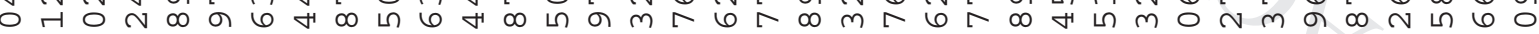

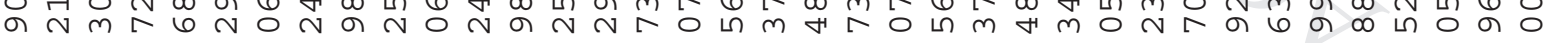
0

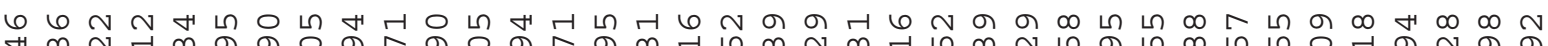
봇

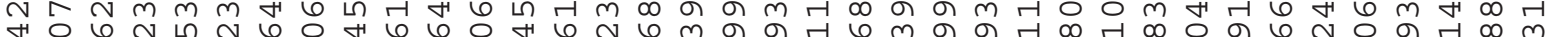

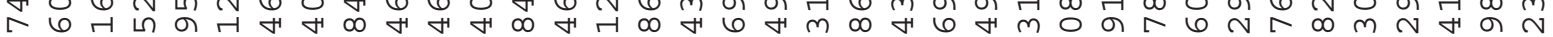
닥

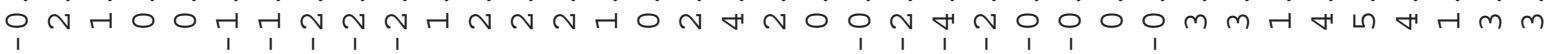

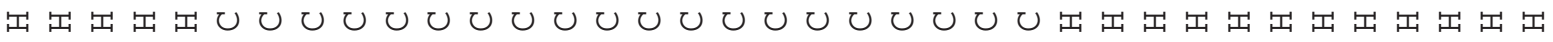
닥 


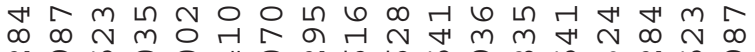

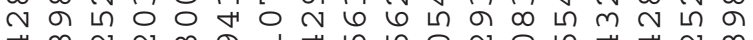
$\forall \infty N N m$ N $N$ 开 К

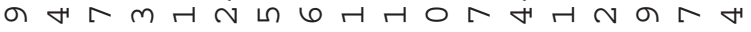

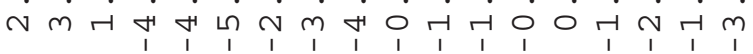

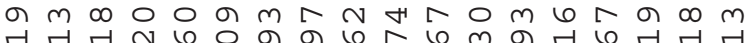
न न

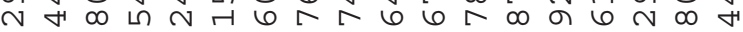
बेतम में

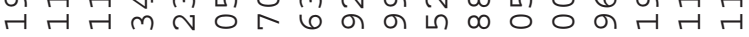

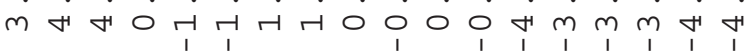

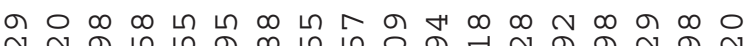

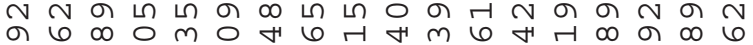

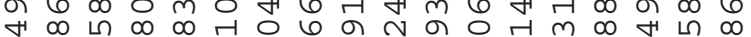

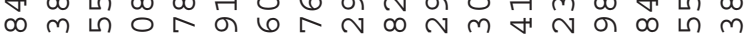

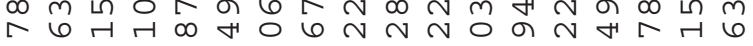

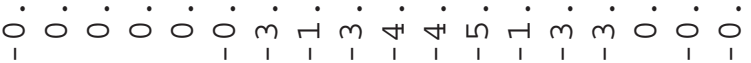

出出出出出出出出出出出出出出出出出出

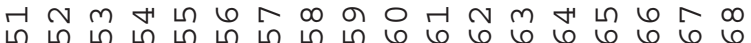

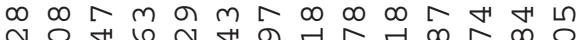

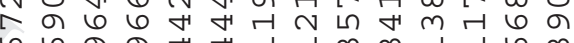
66 の $000 \infty \pi \hat{0}, \begin{array}{lll}0 \\ 0\end{array}$ 0

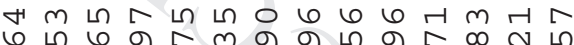
v N 0 H ก बर

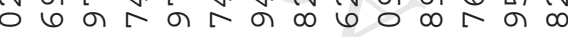

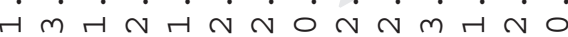

H

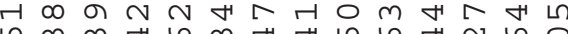
6 ชู $m$ - 0 r $m$ a 6 - $m$

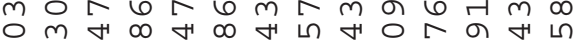

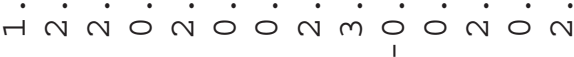

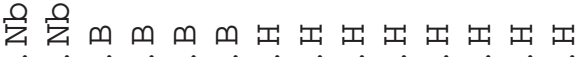

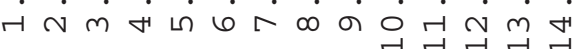




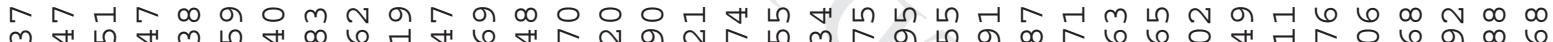

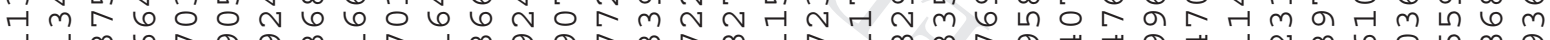

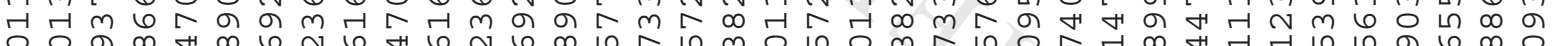
다

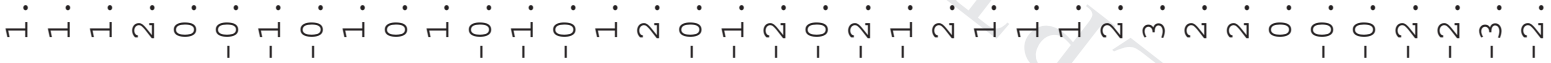

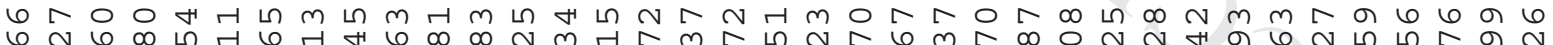

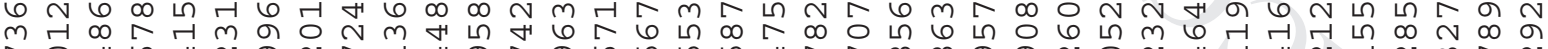

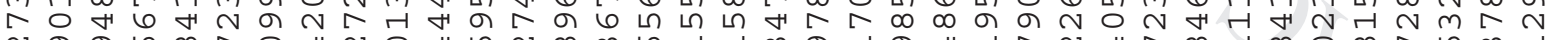

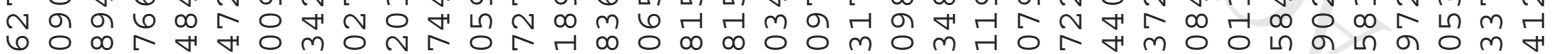

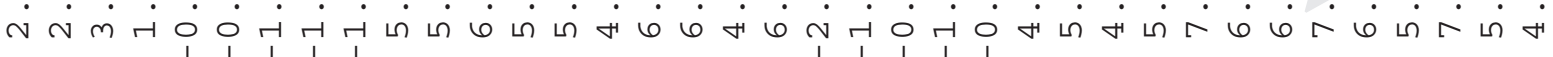

ᄂ เn $N$ ட $m$ t 0 o $m$ m

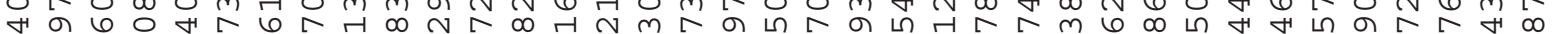
将

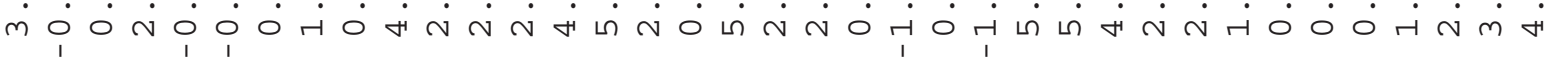

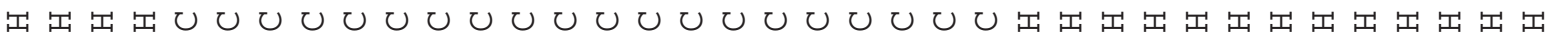

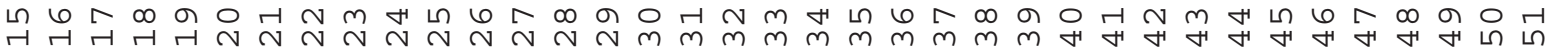




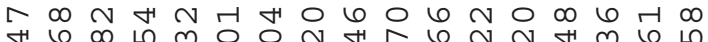

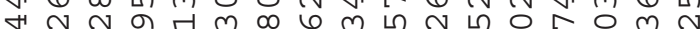

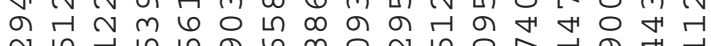

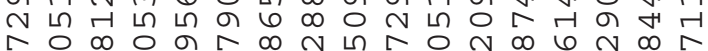

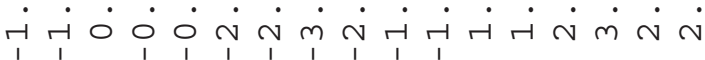

अָ

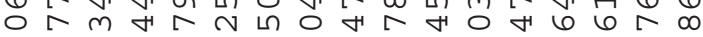

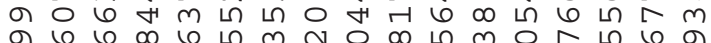

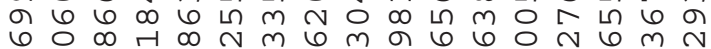
में

N $\infty$ の

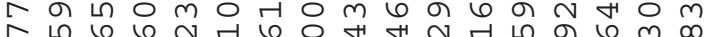
$\sim \sim \sigma \infty$ मी न न न न

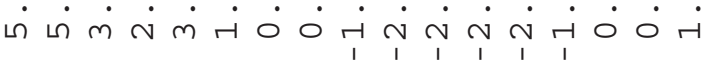

出出出出出出出出出出出出出出出出出

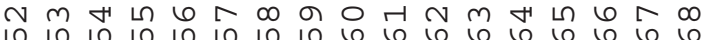

더

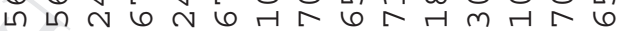

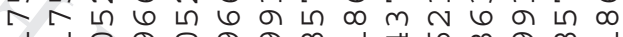

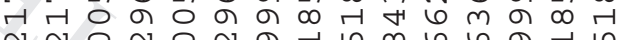
ก

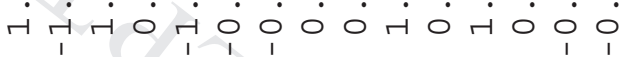

กิก N $N$ ᄂ म म 6 त 6 न ๒ உ 운

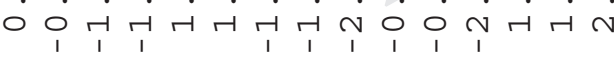

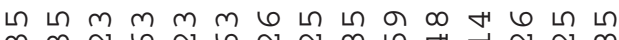

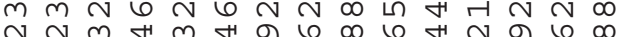

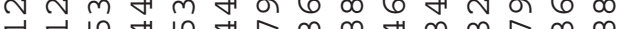

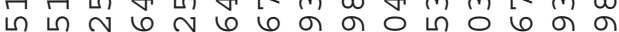

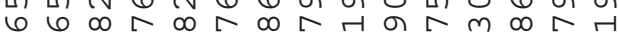

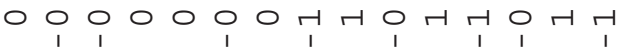

Е

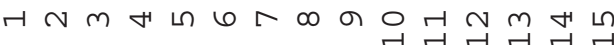




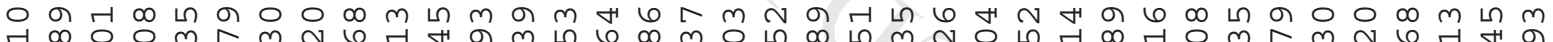

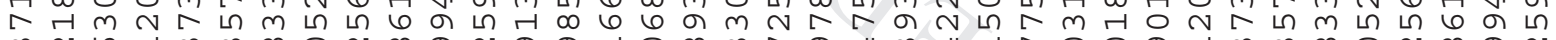

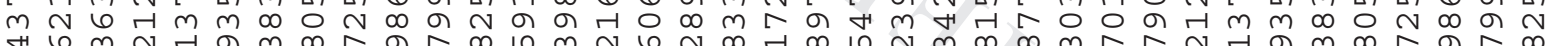

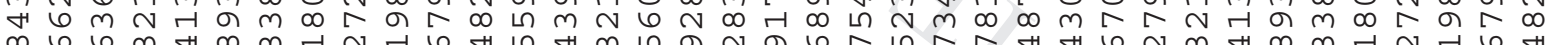

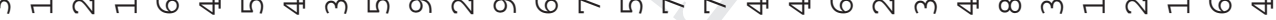

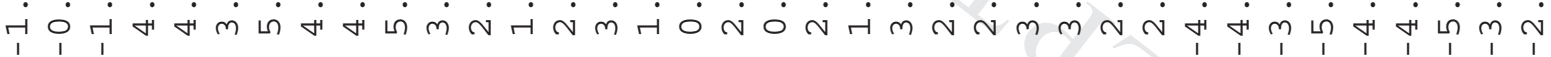

걱 m

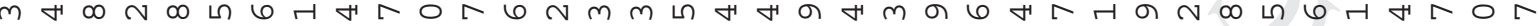

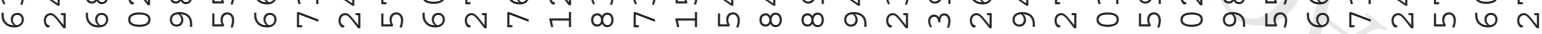

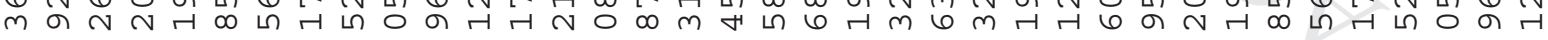
0 0

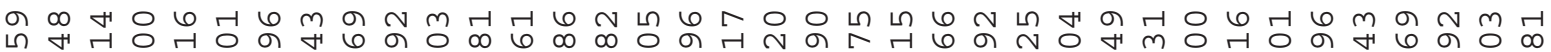
し)

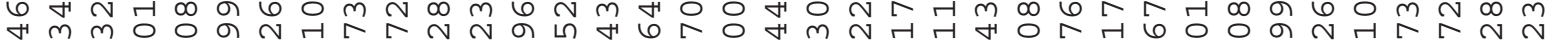
0 ह 0 म

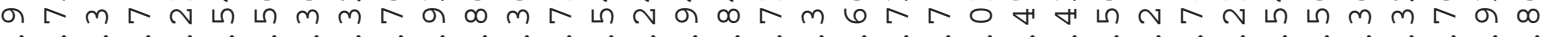

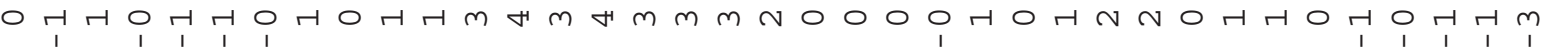

出出出U出出出U出出出U出出出U出出出U出出出UUUUUU出出出U出出出U

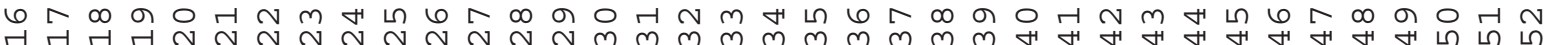




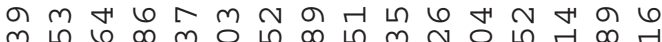

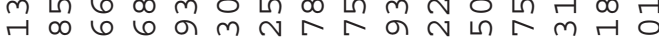
न न

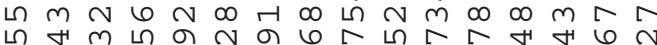

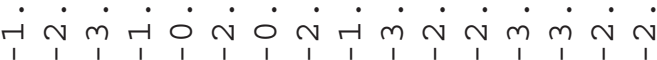

$0 \hat{0} \hat{0}+4)$ $m 6$ เ

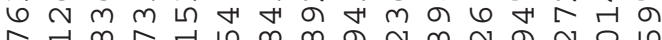

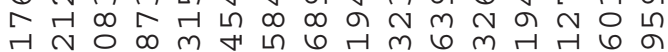

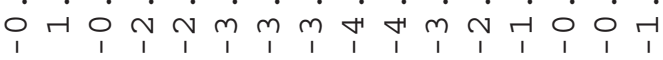

당

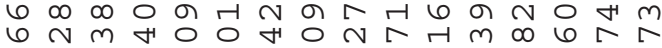

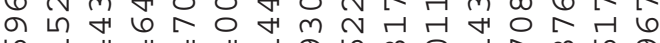

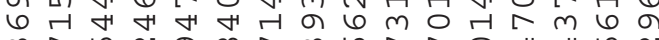
$m$ ก

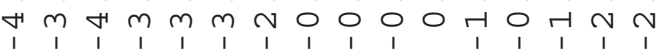

出出出U出出出U出出出UUUUU

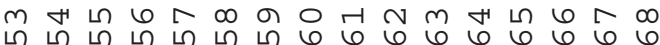

a ब

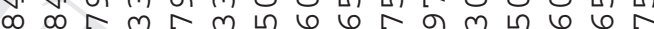

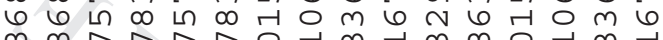

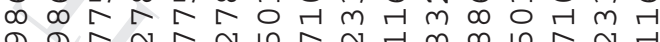

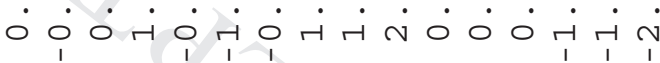

नि

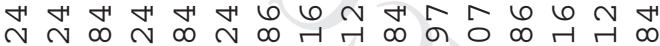
मा मतन 0 L 1 人

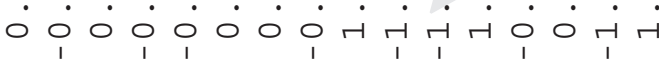

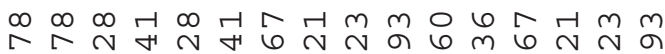

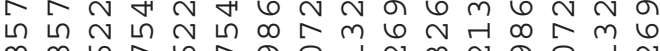

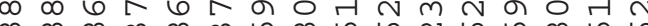

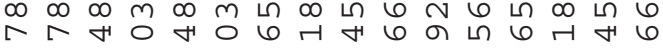
0 o 0 मr

过艺のッ๓๓出出出出出出出出出出

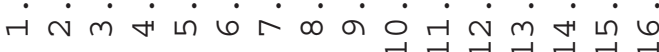


동

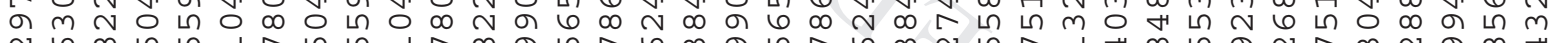

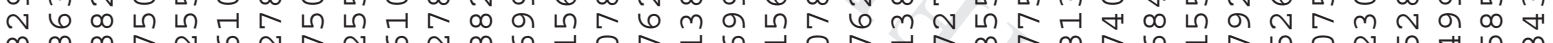

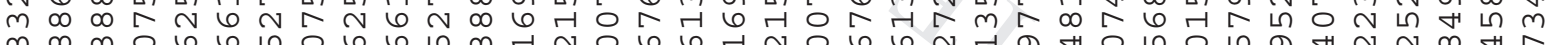

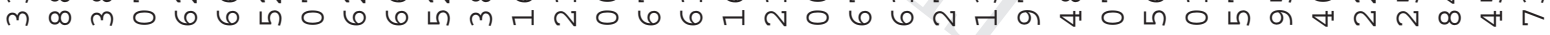

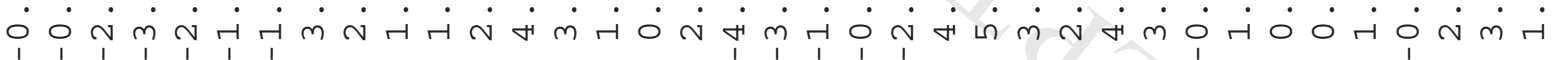

Nㅡ섯

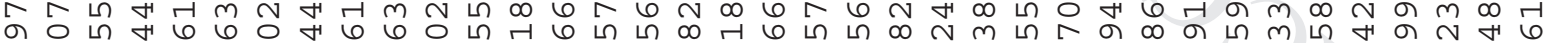
H

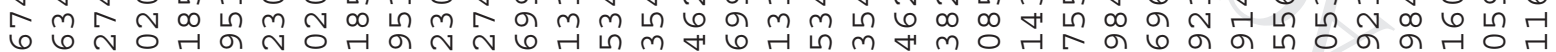

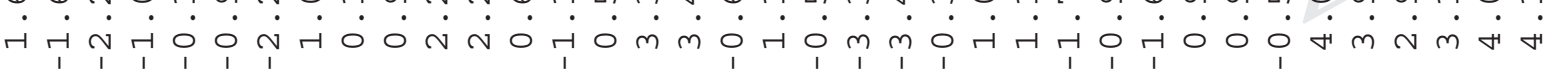

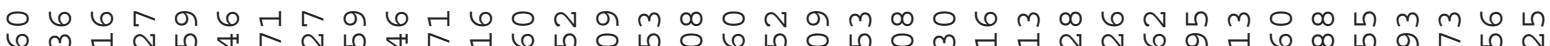

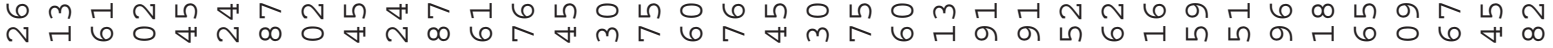

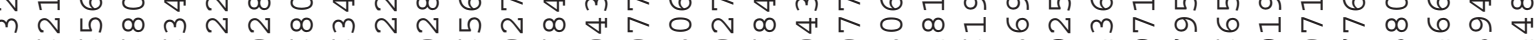
न

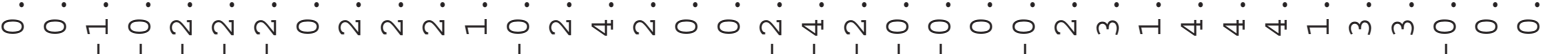

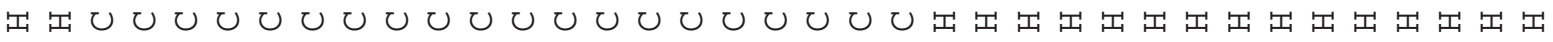

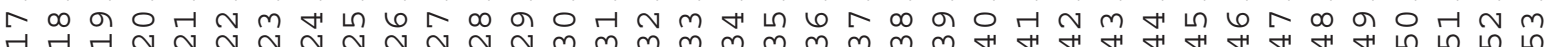




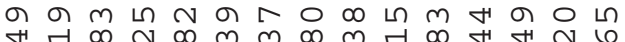

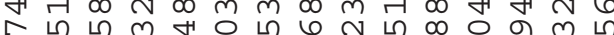
N $\wedge+\infty$ म

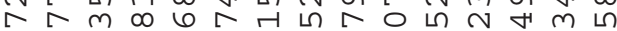
N

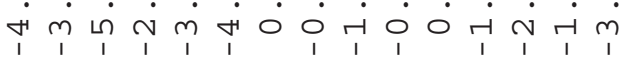

군 サ ம त 6 म 6 म

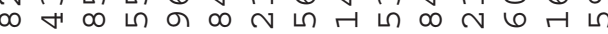
m

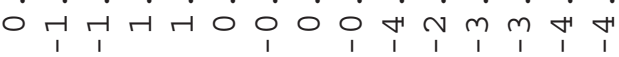

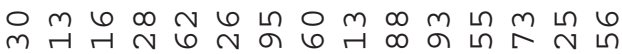
m $\infty$ ชै + $\infty$ б̆ स

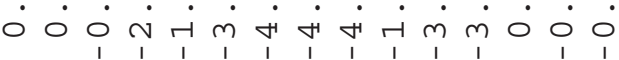

出出出出出出出出出出出出出出出

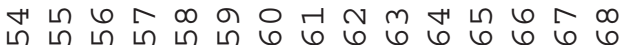

ம்

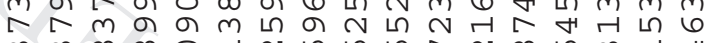

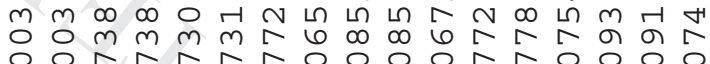

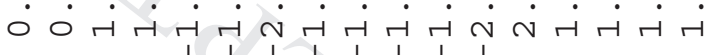

$\infty$ の

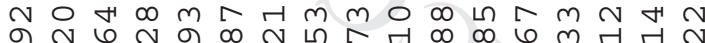

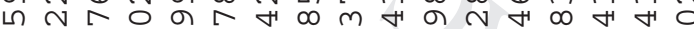
स 1 r 0 a

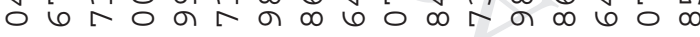

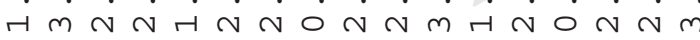

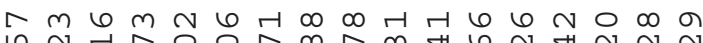
$\llcorner N$ ก 6
0

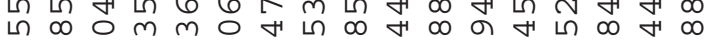

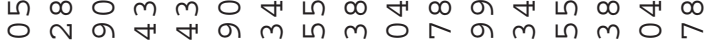
$\dot{r} \dot{\sim} \dot{0} \dot{N} \dot{0} \dot{0} \dot{N} \dot{m} \dot{0} \dot{0} \dot{0} \dot{N} \dot{m} \dot{0} 0$

$\sum \sum \cong \sqcap \sqcap$ 出出出出出出出出出出出

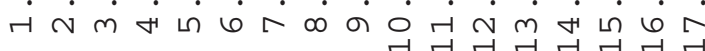


늠

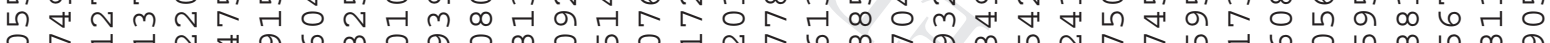
O

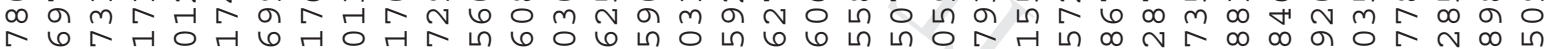

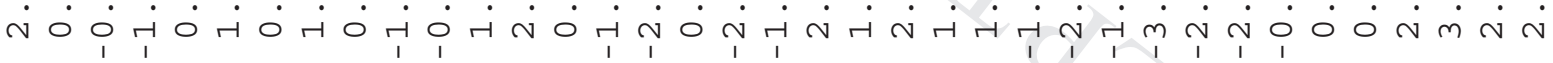

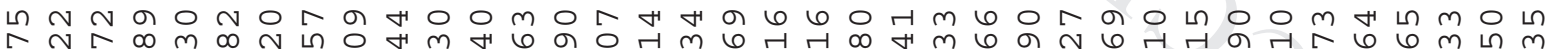

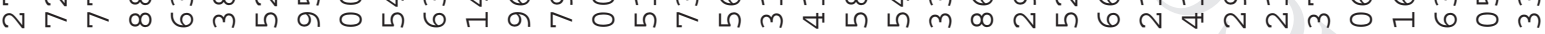

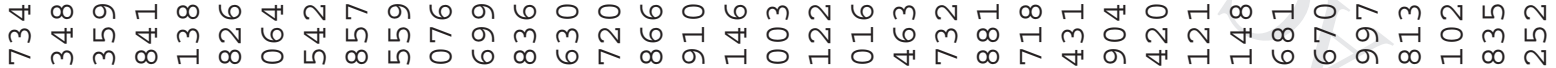

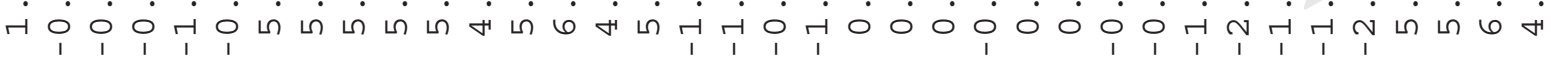

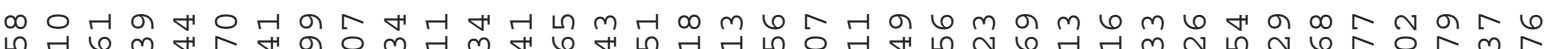
싱

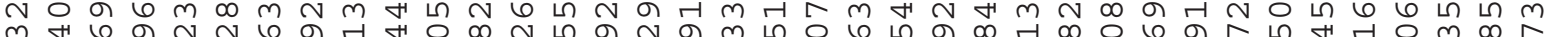

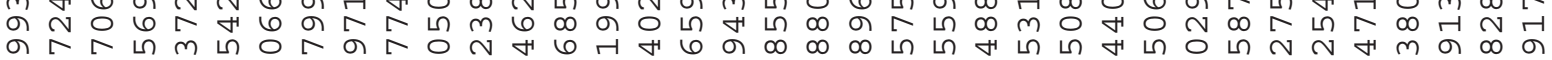

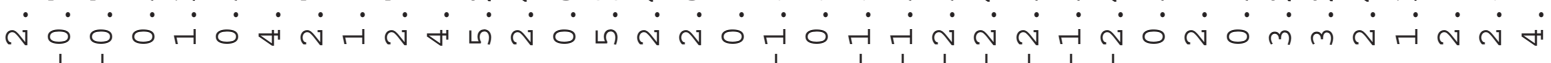

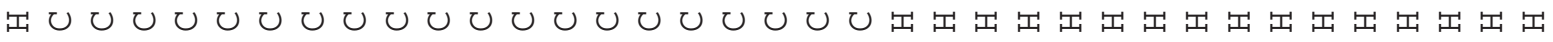

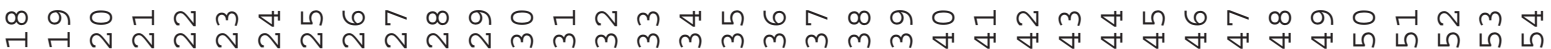




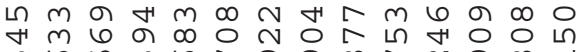
$m$ b $m b 0$ th $10 m \in 6 \pi \infty$

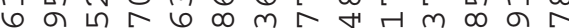

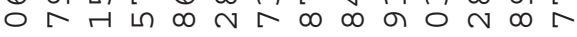

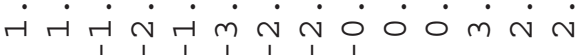

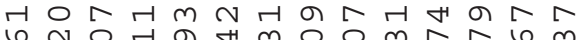

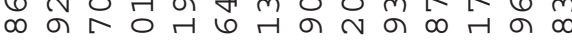

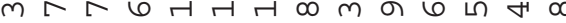
$\infty$ б न $\infty N$ स ब मी ब $N$ G

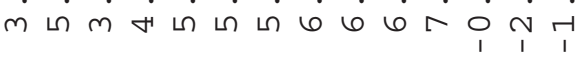

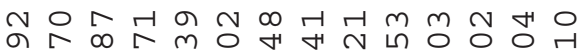
बิ

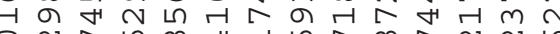

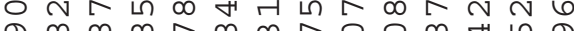

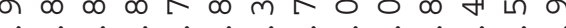
ก

出出出出出出出出出出出出出出

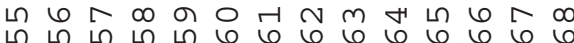

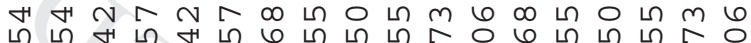

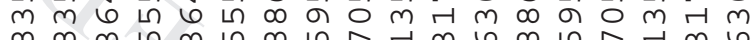

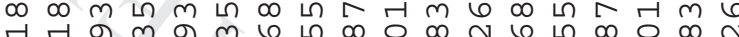

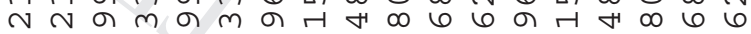

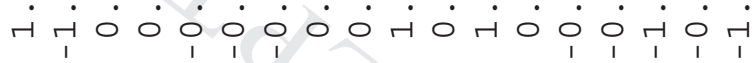

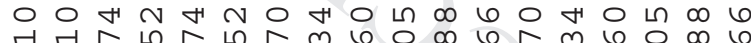
a वे अ म

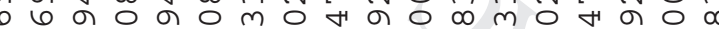

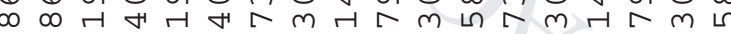
H H $r$ H

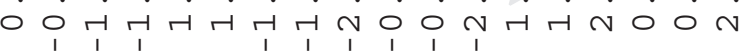

人⿵内人 மூ

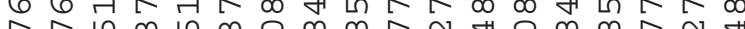

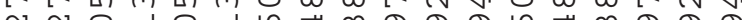

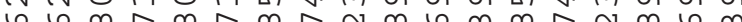
$\begin{array}{ccc}0 \\ 0 & \infty & 0\end{array}$

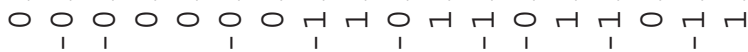

ろろッறறの出出出出出出出出出出出出

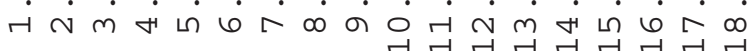




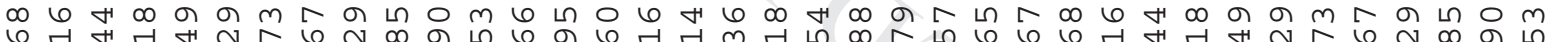

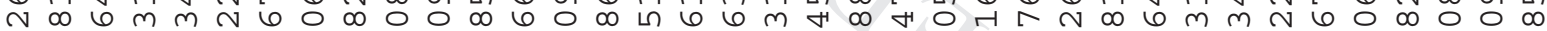
NON N

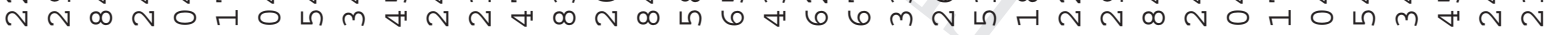

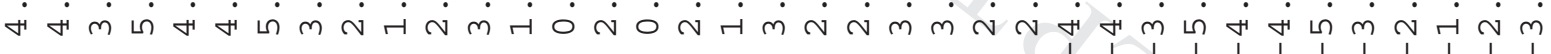

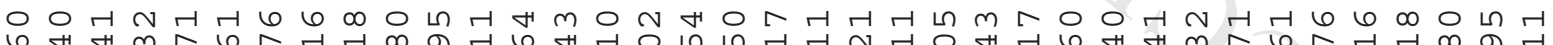

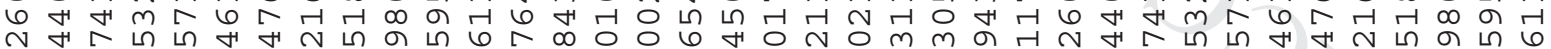

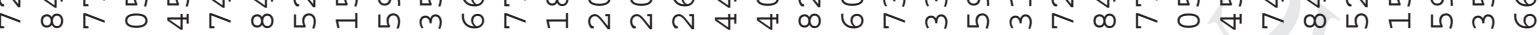

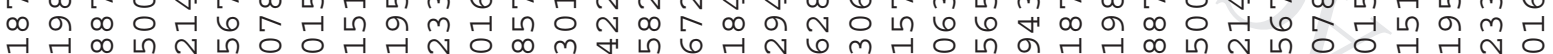
ت 0 0

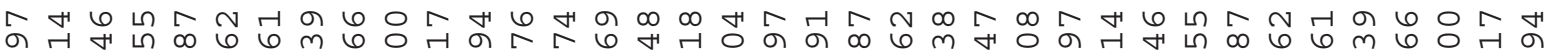

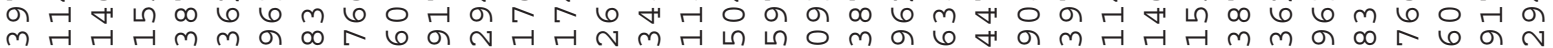

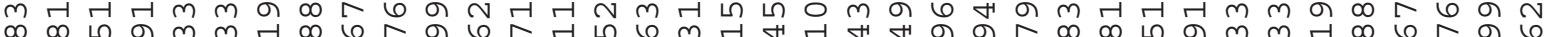

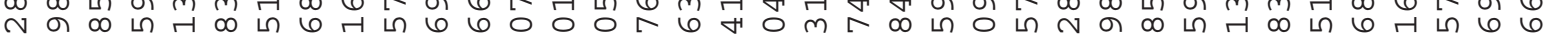

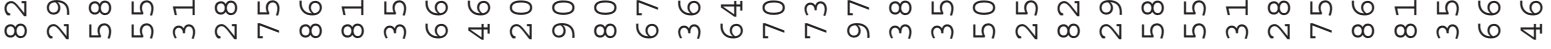

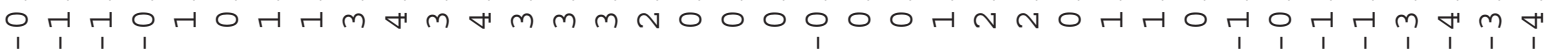

U出出出U出出出U出出出U出出出U出出出UUUUUU一兀出出出U出出出U出出出

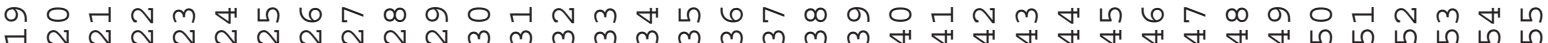


மேிำ 60000 $m m$ म $\infty$ ब

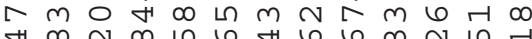

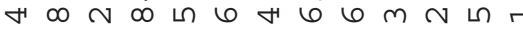

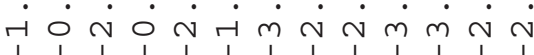

4mon 하성

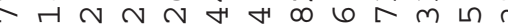

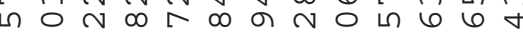

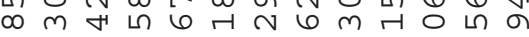
$\dot{v} \dot{m} \dot{m} \dot{m} \dot{\gamma} \dot{m} \dot{\sim} \dot{0} 0 \dot{0}$

c

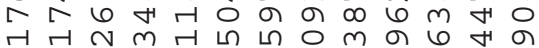

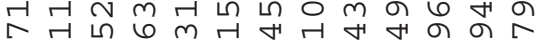
○ 00060 ㄱํㅇ

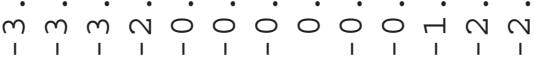

U出出出U出出出UUUUU

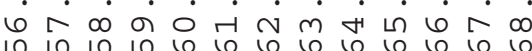

ஸ $\infty$ ह 6 -

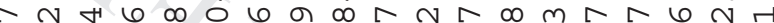

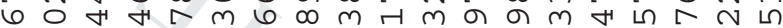

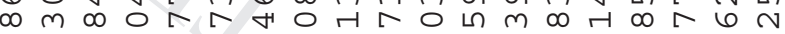

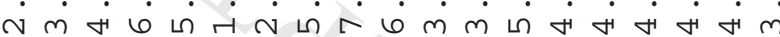

m

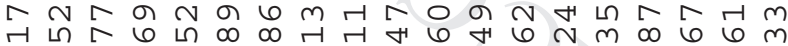

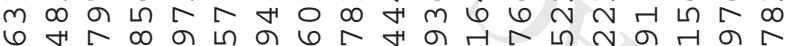

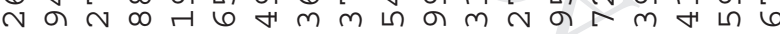
மम

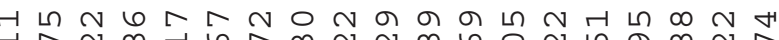

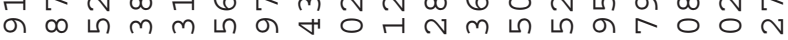

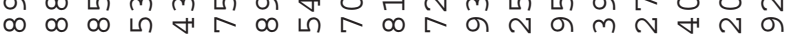
$\infty \infty$

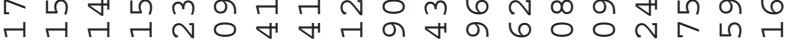

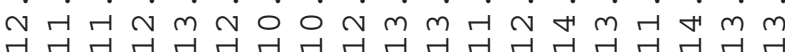

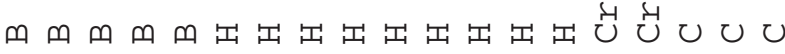

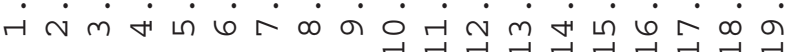


เூ

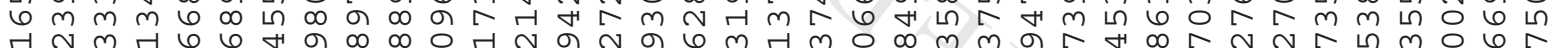

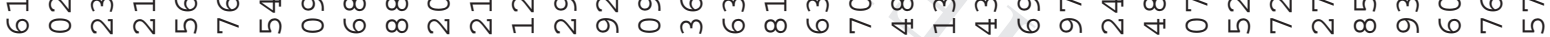

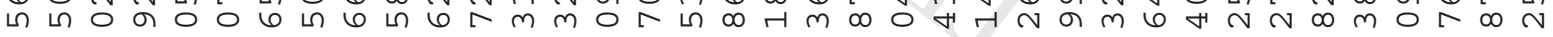

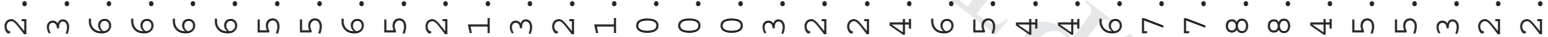

m

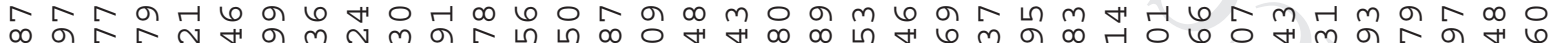

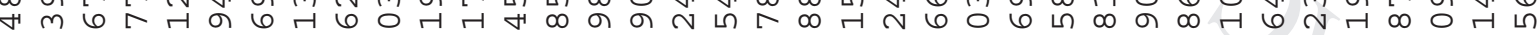
மீ유

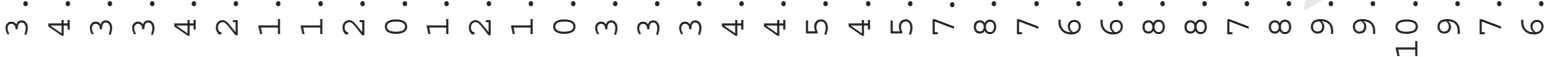

ঋ

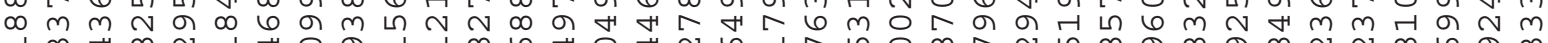

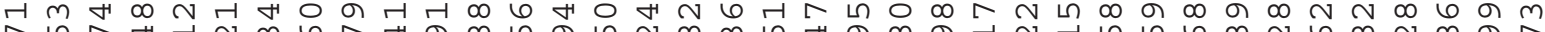
今。

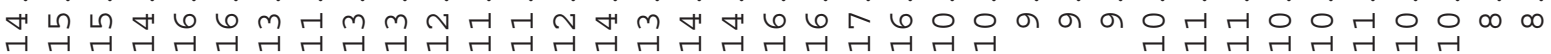

UUU出出出U出出出U出出出U出出出U出出出UUUUUU出出出U出出出U出

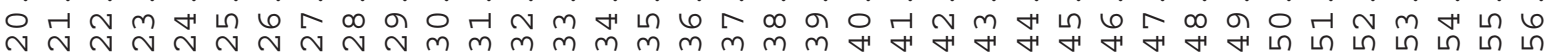


$\infty$ 6 a $\Omega \infty \infty$ $\circ m+6 N N$ r $60 \infty$ $\infty \pi \sim r+106 \sim m \sim$ $m \infty$ เी 0 \% $\dot{\sim} \dot{\sim} \dot{\sim} \dot{m} \dot{4} \vec{\sim} \dot{\sim} \infty$

$6 \sim m \sim \circ\llcorner 6 \infty \sigma$

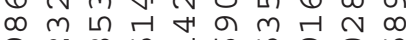

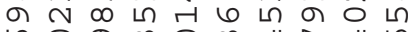
$60 \%$ m $m$ H

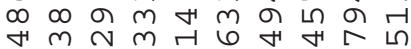
$\infty \infty$ ம்

$N$ H

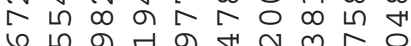

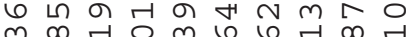
$m \infty$ r $m$ m

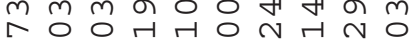
の $\infty \infty \infty \curvearrowleft \propto \infty \stackrel{\circ}{\circ}$

出出U出出出U出出出 กิ
N m m

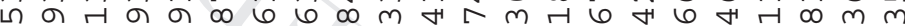

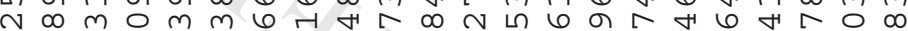

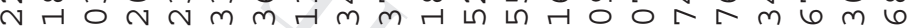
00000000000000 H $N$ in $6 \mathrm{~m} N$

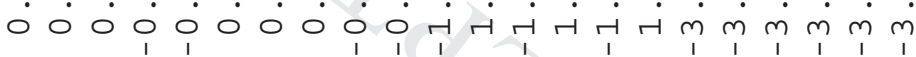

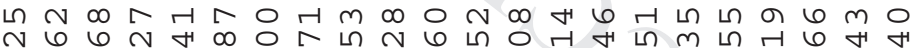

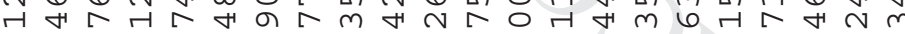
살

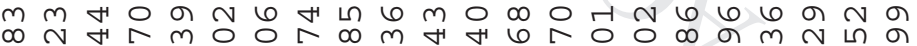
ت

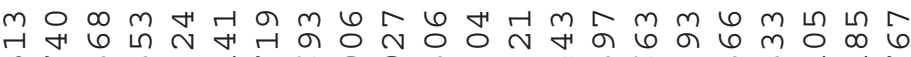

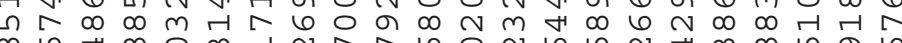

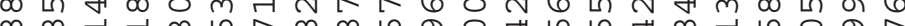
$\infty \infty \cdots$ $m, n$ h

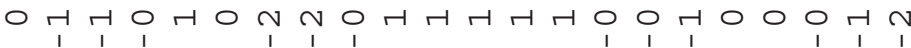

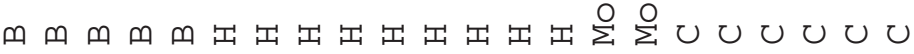

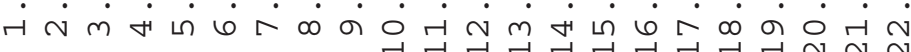


당 G

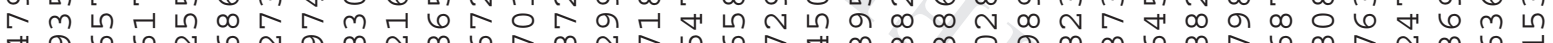

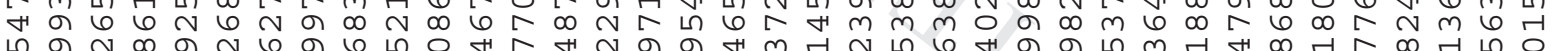

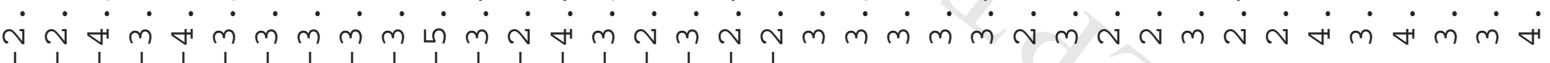

것으 $m 0 \infty$ ก 00 ద

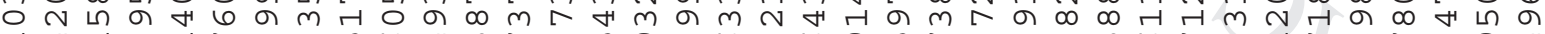
걱

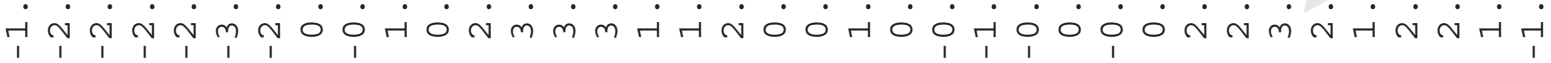

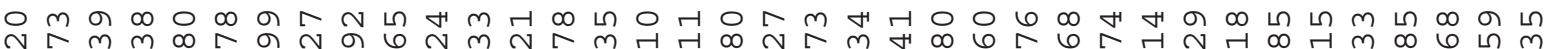

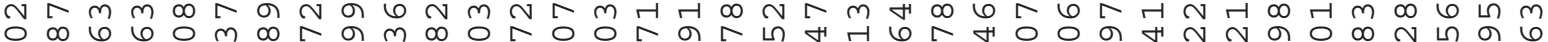

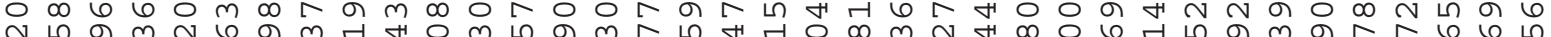

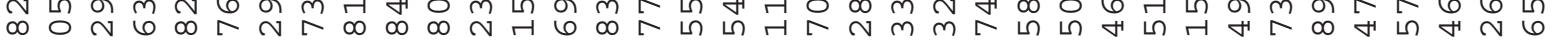

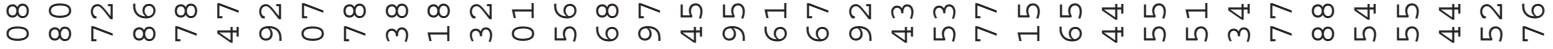

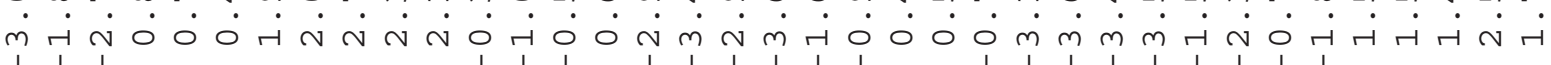

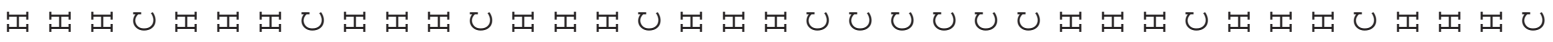

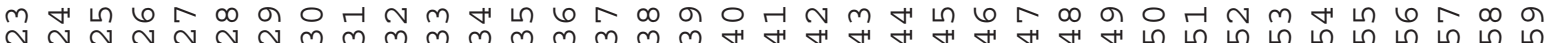




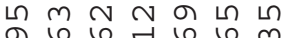
N $m-1060$

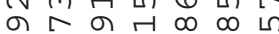
$\circ$ เ $\infty \mathrm{m} 66$ - 6 เी เी $N \infty$ เी மं $\dot{m} \dot{m} \dot{m} \dot{H}$

4

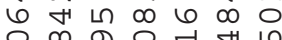

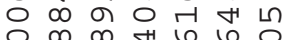
$\circ \infty \infty \begin{array}{llll}0 & 0 & 0\end{array}$

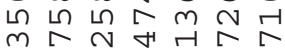
- $0 N m \sim N$

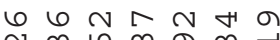
$N m\llcorner\infty m$

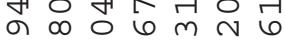

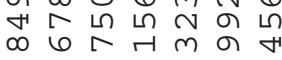

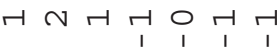

出出出已出出出

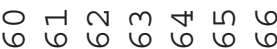

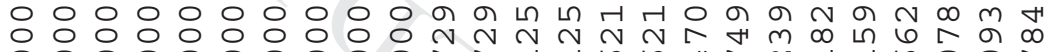

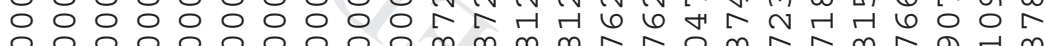
年

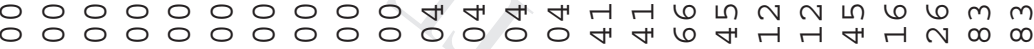

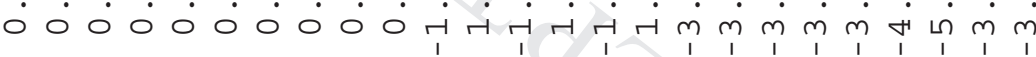

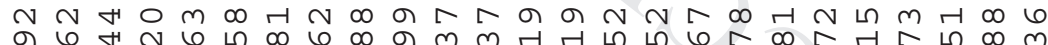
बे

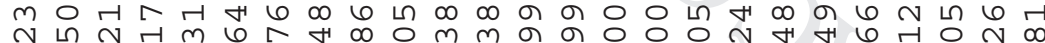

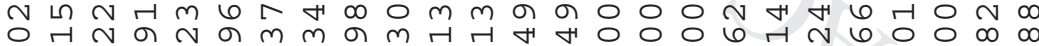

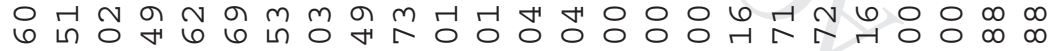

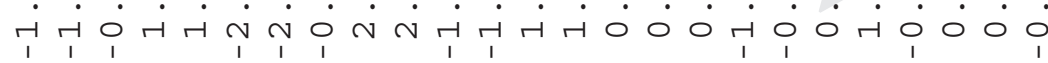

ॠ

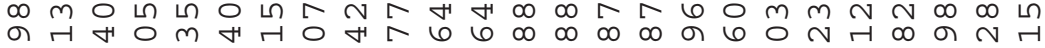
1)

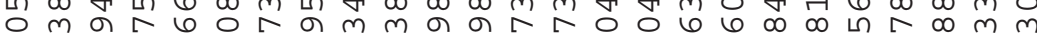

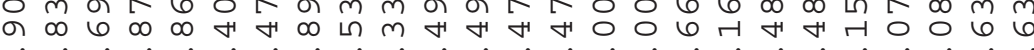

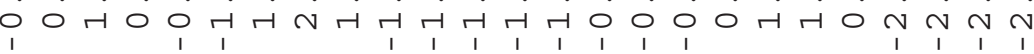

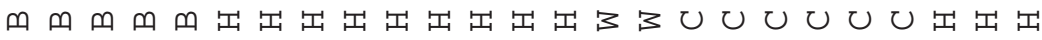

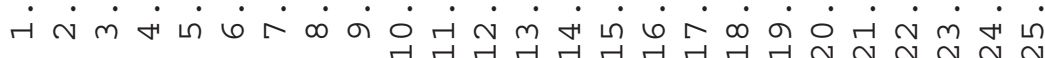


웅

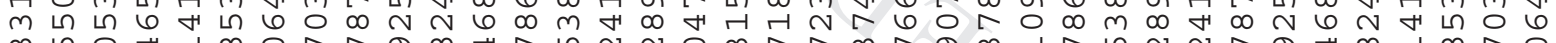

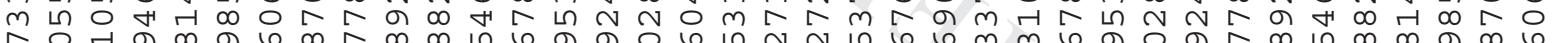

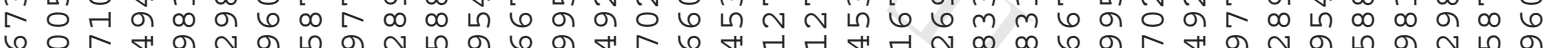

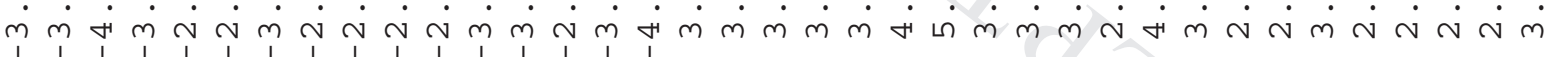

サய ○ में

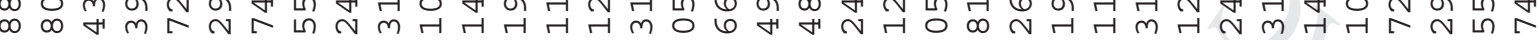

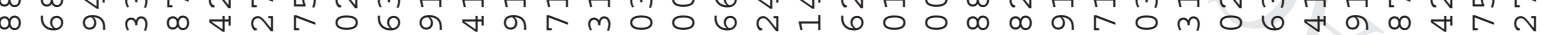

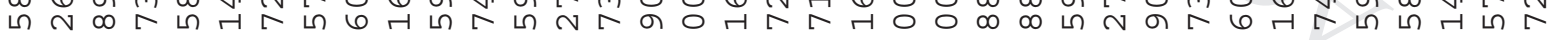

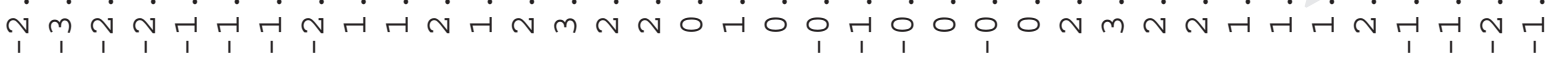

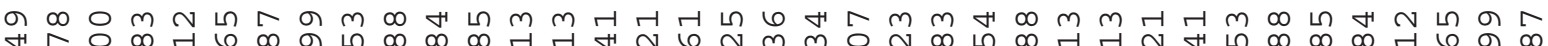
サं H त ヘn

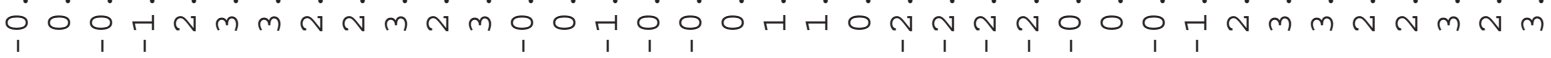

U出出出U出出出U出出出U出出出UUUUUU出出出U出出出U出出出U出出出

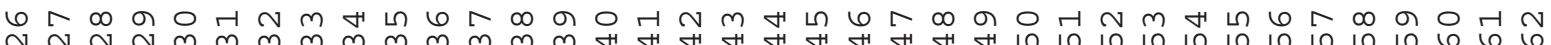


움요 $m$ เก 6 เก $m$ मी ल० ने $\dot{m} \dot{m} \dot{m} \dot{\gamma}$

$\begin{array}{llll}4 & 4 & 0 \\ 4 & 0 & 0\end{array}$ $\infty$ o व $m$ $\infty \quad \infty m+$ $\infty 6 \mathrm{~m}$ a เ $\sim \wedge \infty$ $\underset{1}{\sim} \dot{m}_{1} \underset{1}{\sim} \underset{1}{\sim}$

or $\infty \mathrm{m} 0$ अ 구 $m$ v

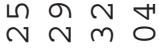
$0 \begin{array}{lll}1 & 0 \\ 0 & r_{1} & 0\end{array}$

U出出出

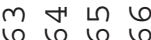

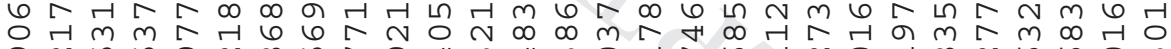

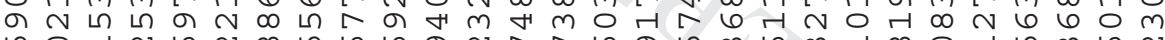

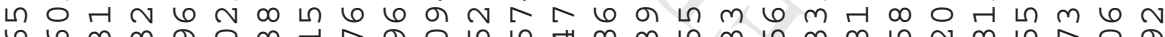

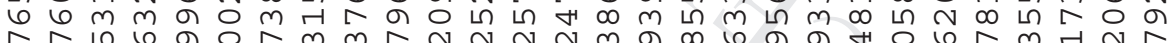

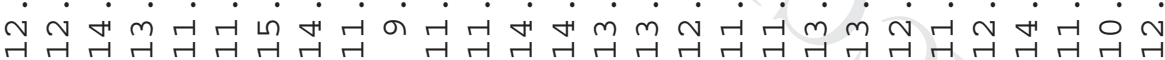

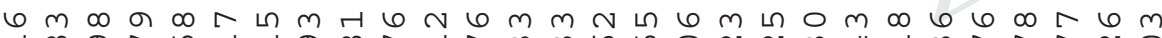

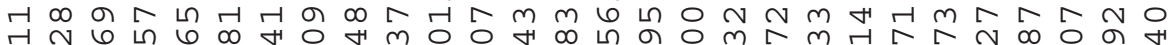

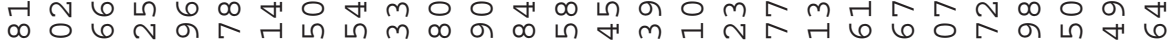

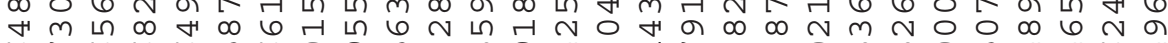

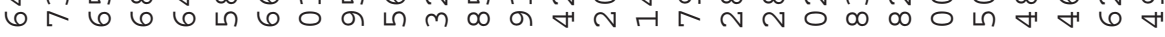
6 H

다

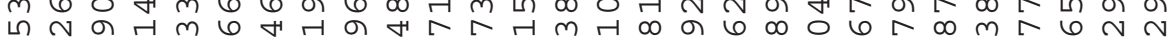

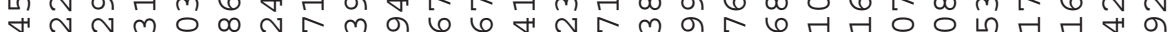

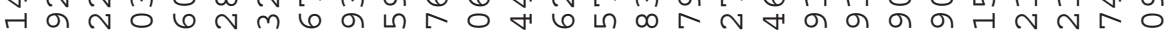

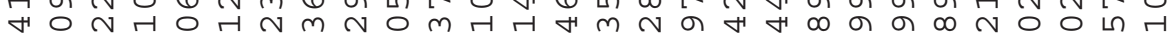

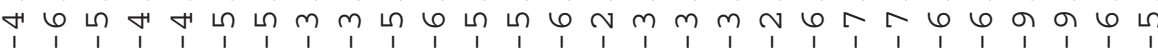


向

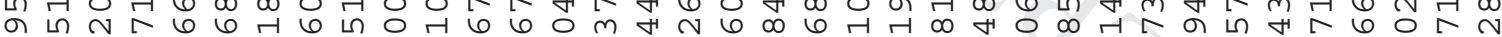

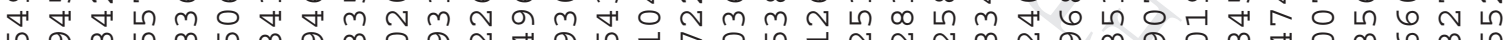

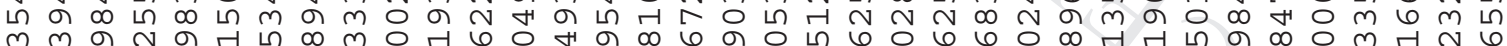

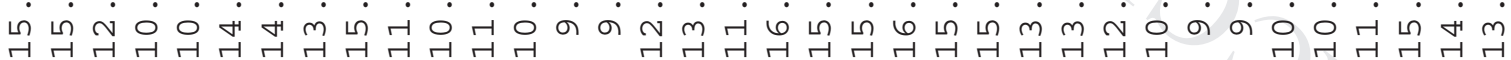

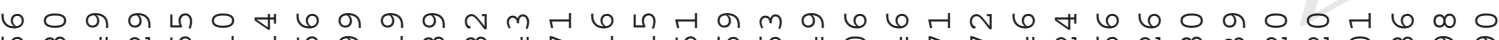

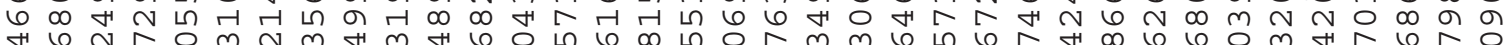

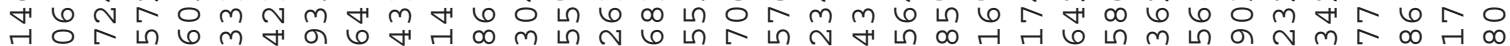

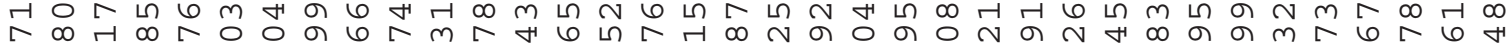


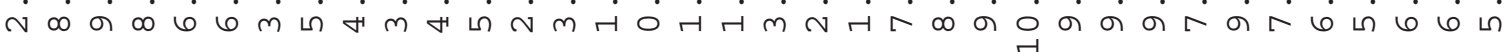

ดิ

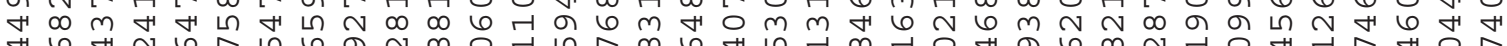
H 6 H

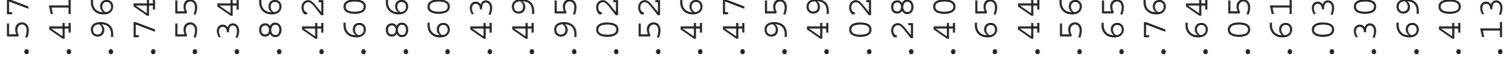

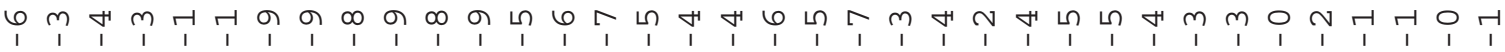




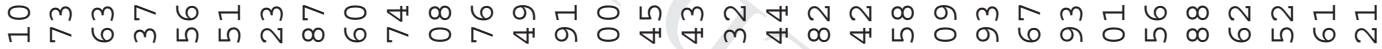

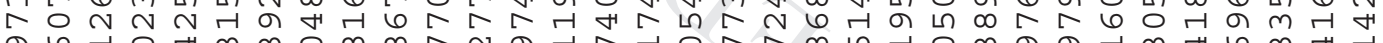

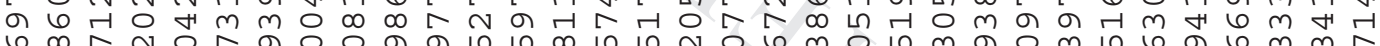

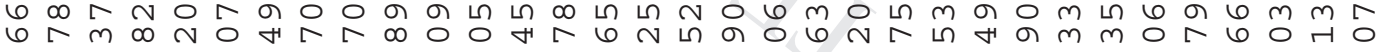

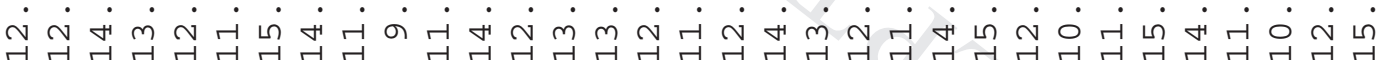

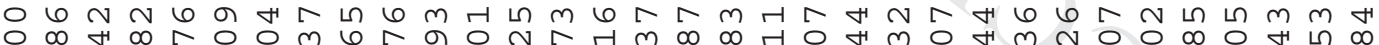
b人

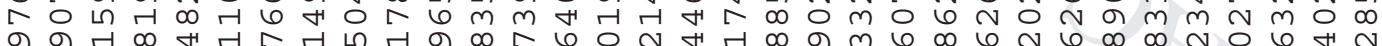

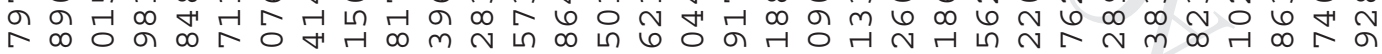

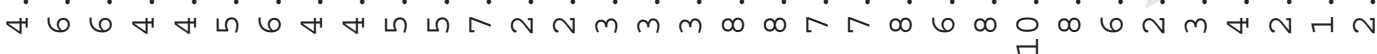

ナூ

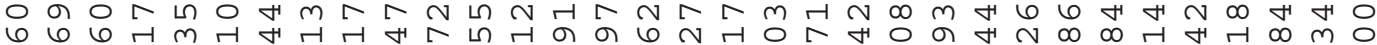

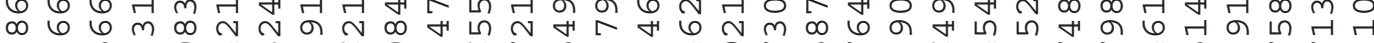

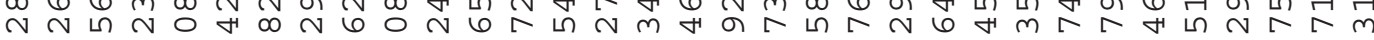
$m$ เก

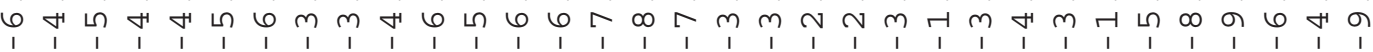

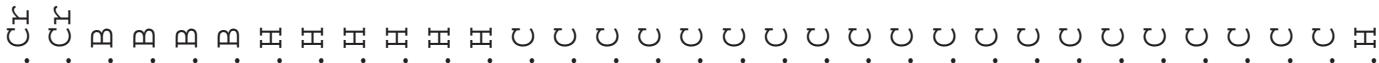

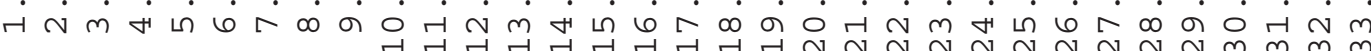


光

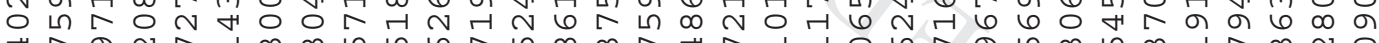

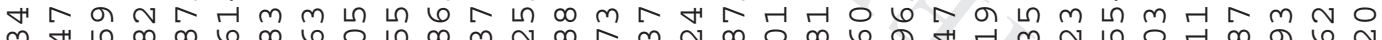

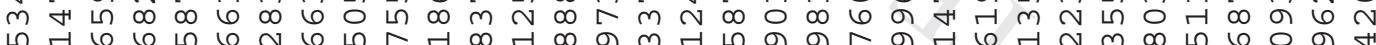

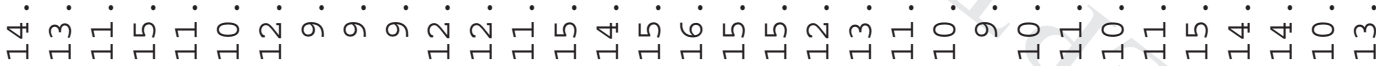

H

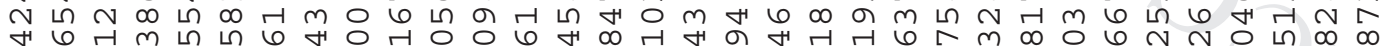

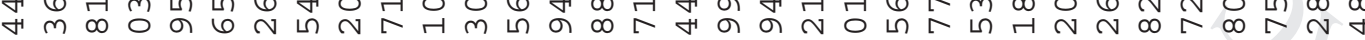

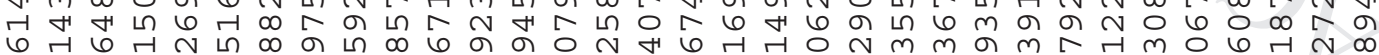

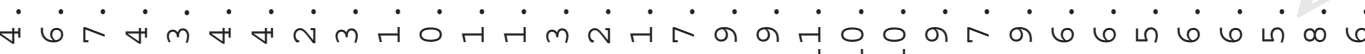

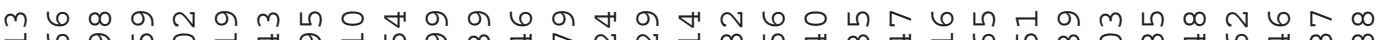
r

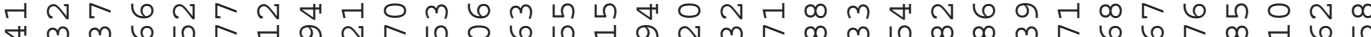

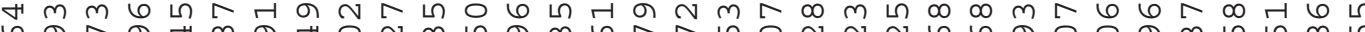
6 月 H

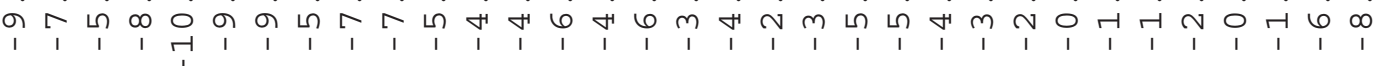

出UU出出出出出出出出出出出出出出出出出出出出出出出出出出出出 0 ○

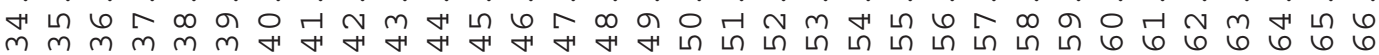




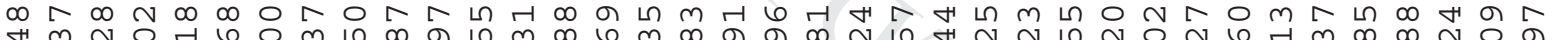

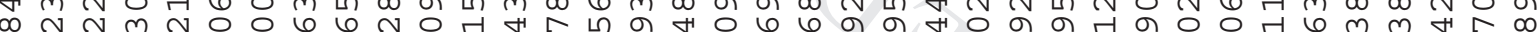

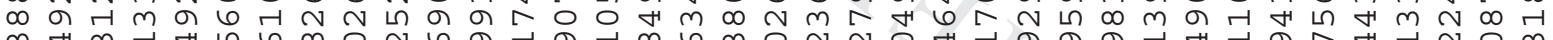
m

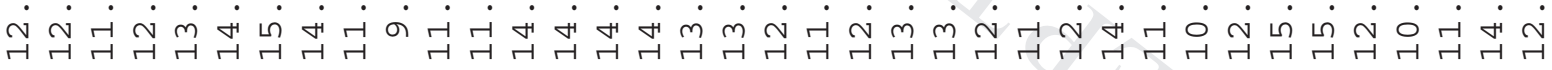

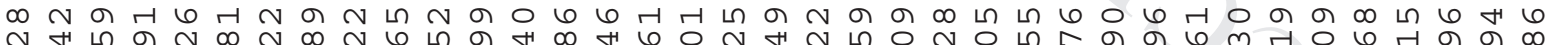

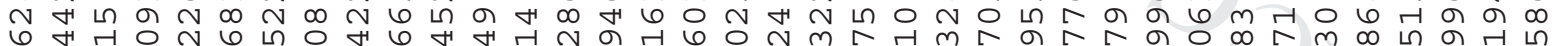
H

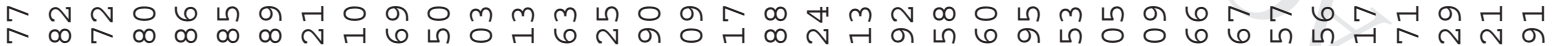

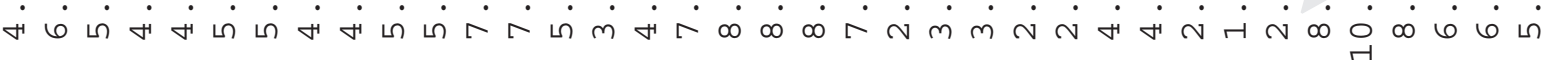

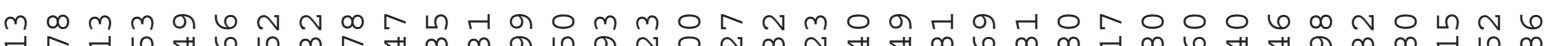

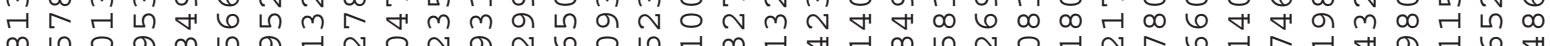

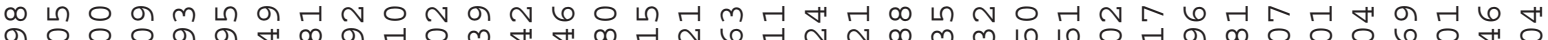
o $\begin{gathered}0 \\ 0\end{gathered}$

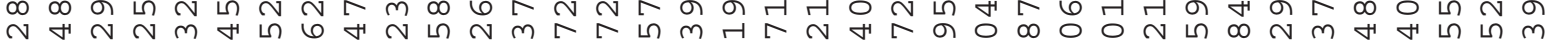

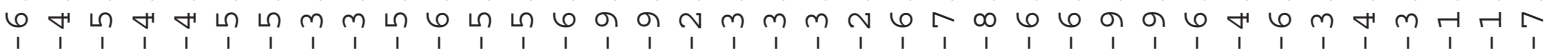

Uூ

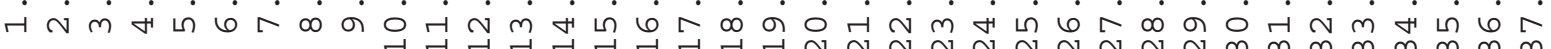


ᄂ

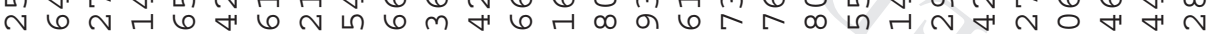

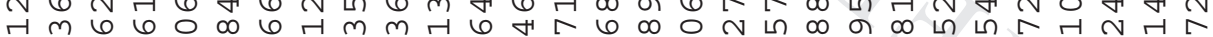

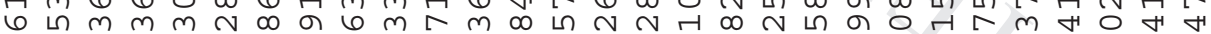
6 अ $\mathrm{m} \infty$ の

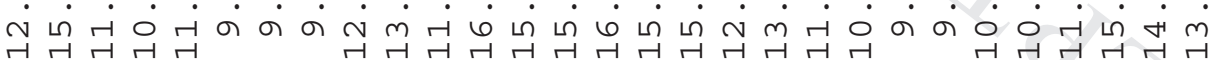

$\infty \sim \infty$ ○ ก) เी $m$ in

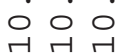

H HO เ

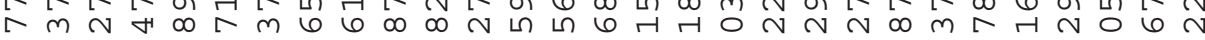

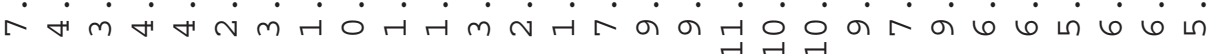

光 0 i

เก $m \infty$

เि

가 $\stackrel{\circ}{\sim} \stackrel{\infty}{1}$ $\therefore \dot{6}$

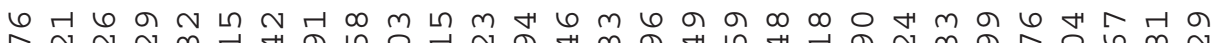

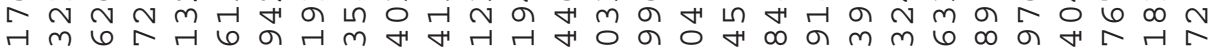

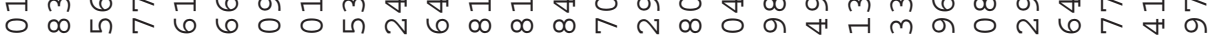
+人 सं

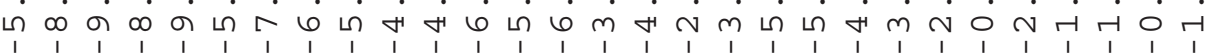

$\operatorname{con}$ H $\sim \infty$ o $\circ 66$ m $6 \mathrm{~m}$ 0 - 14

出出出出出出出出出出出出出出出出出出出出出出出出出出出出出 $\sqcap \sqcap \sqcap$

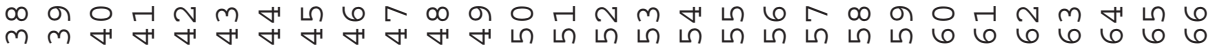




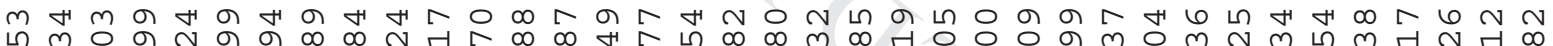

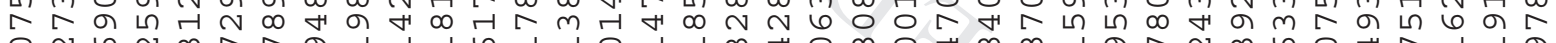

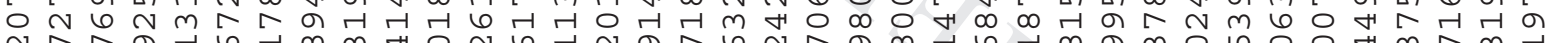

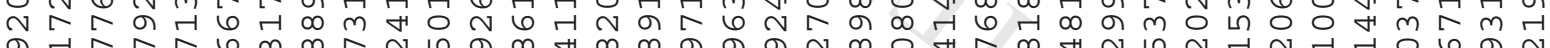

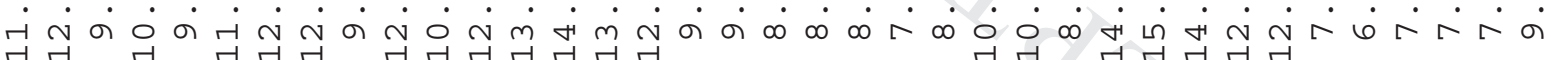

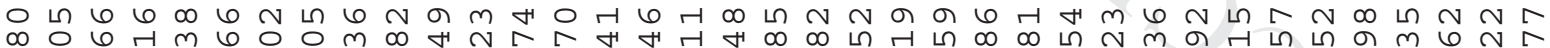

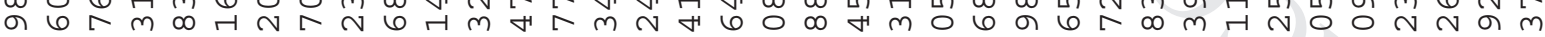
ब 46 H

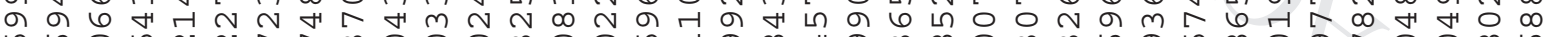

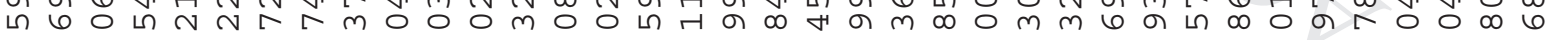

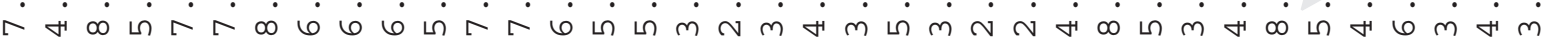

H

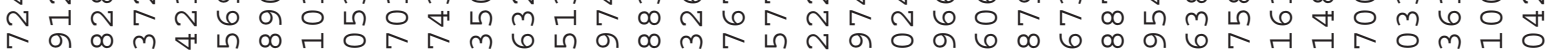

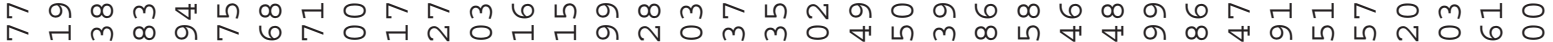

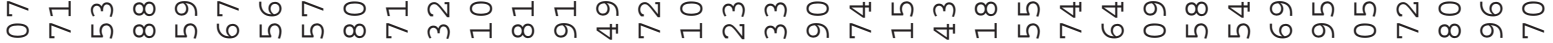

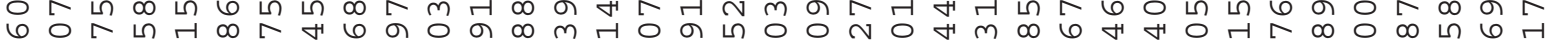

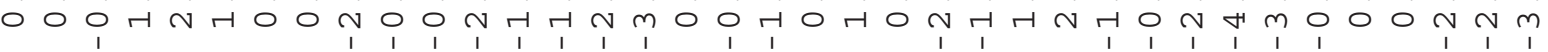

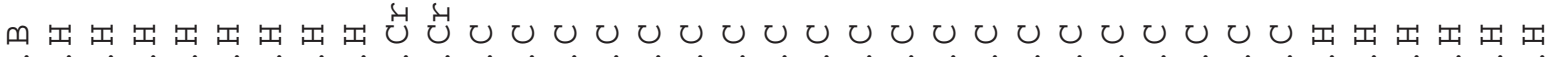
अ 
の $\circ$ 이 N எै

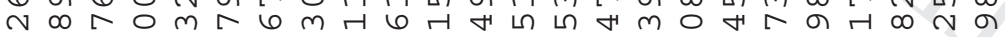

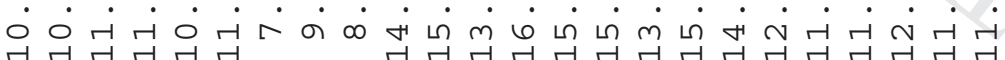

œ बर

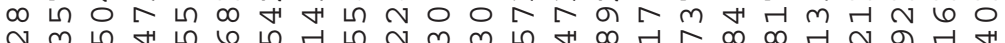

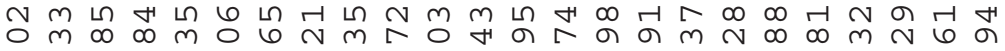

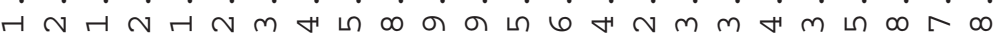

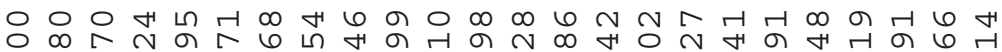

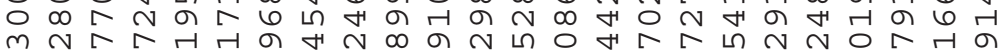
m मा $\infty$ ने

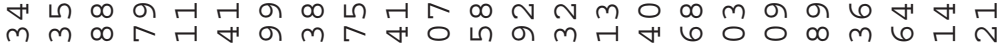

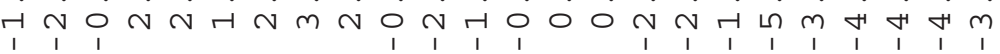

出出出出出出出出出出出出出出出出出出出出出出出出

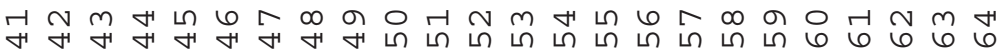

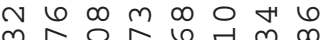
a $6 \sim$ म 4 म $m$ म० $m \infty$ เ $N$ เ $m$ के - $の$ เ 0 \% 6 r

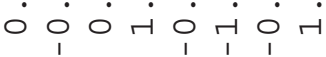

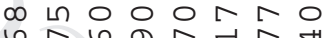
๘ก

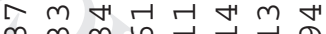

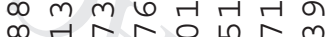

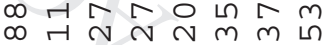
0 or 0 or म

$\sim m \circ m \sim m \sim \infty$ 유 ॠ म० म다의

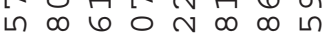

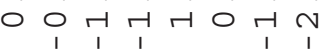

岂岂の तं $m+10 \wedge \infty$ 
H

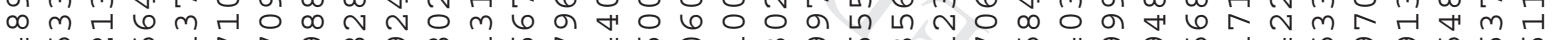

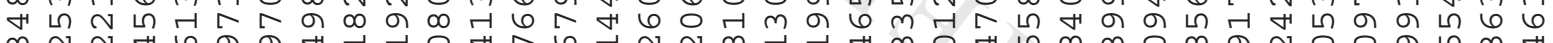

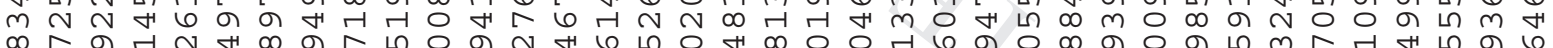

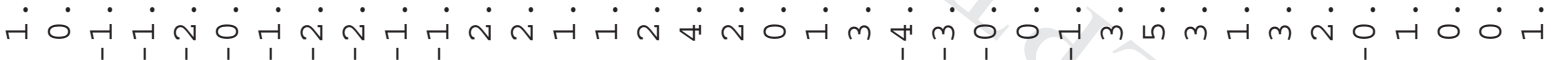

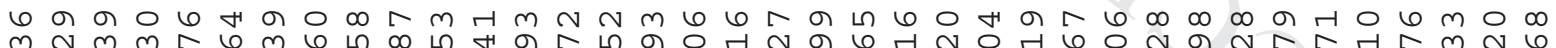

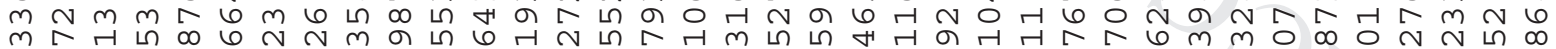
$6 m \infty$ म பก 0 म

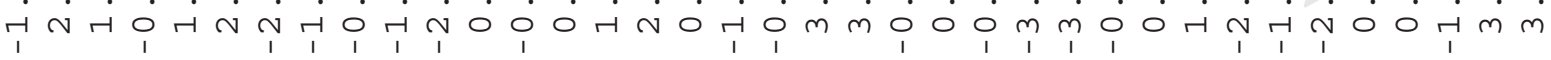

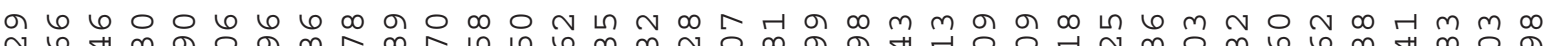
ชै

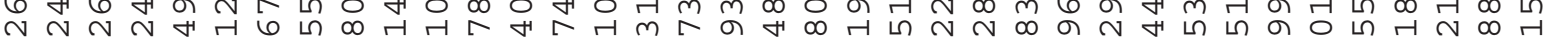

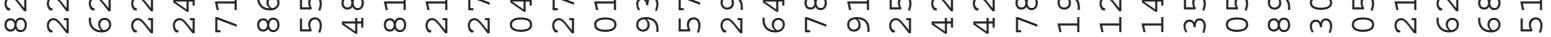

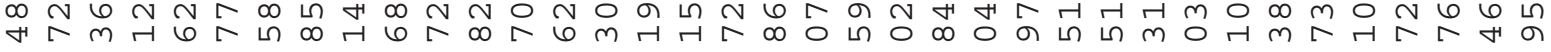

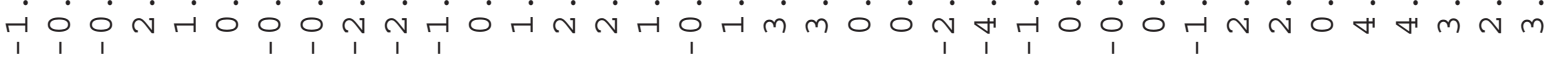

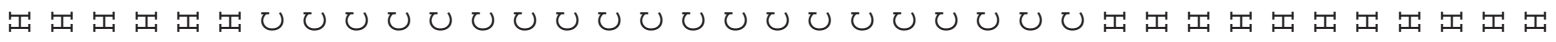

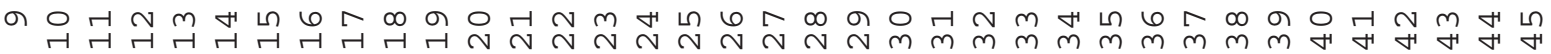




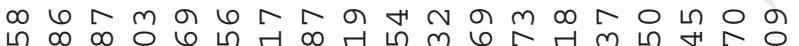

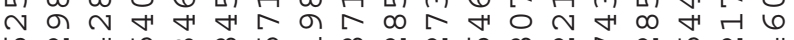

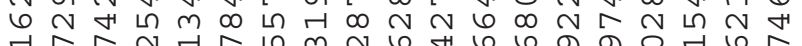

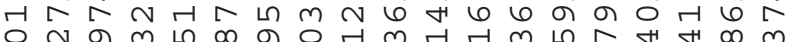
$\dot{\circ} \dot{m} \dot{\sim} \dot{m} \dot{m} \dot{\gamma} \dot{0} \dot{H} \dot{0} \dot{0} \dot{0} \dot{0}$

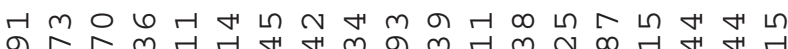

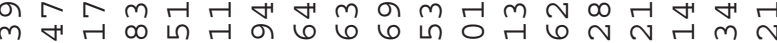

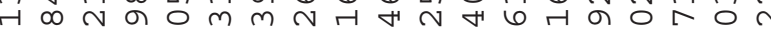
ब담 v m m

舟

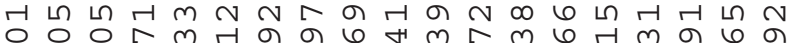

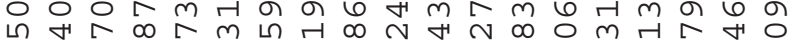
年 m 0 r

出出出出出出出出出出出出出出出出出出出

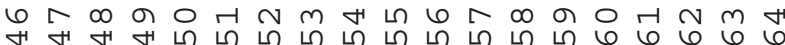

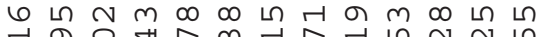
H

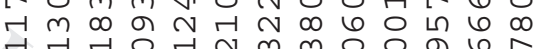

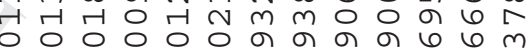

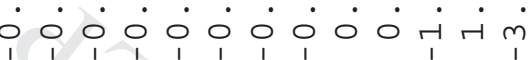

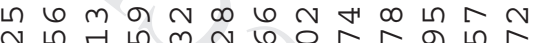
$m$
$m$

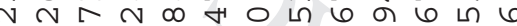
ब $6 m \infty$ म $\infty$ r $\infty 0 \infty \pi n$

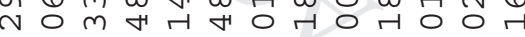

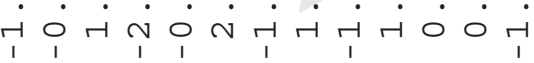

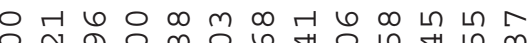

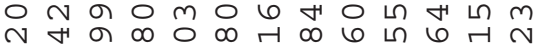
भ

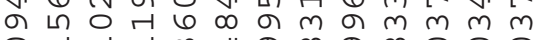
年

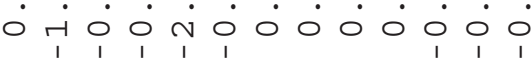

๓๓๓出出出出出出出U

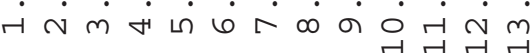




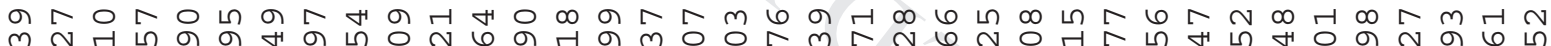
H

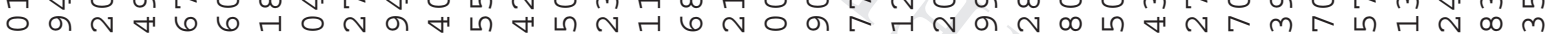
$m \circ m-\infty$ ब

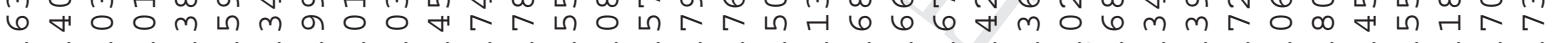

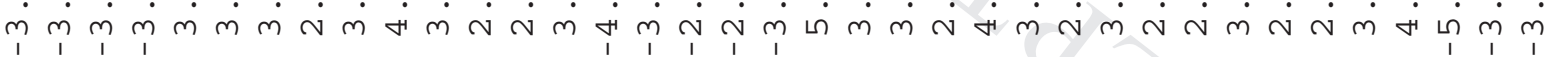

H

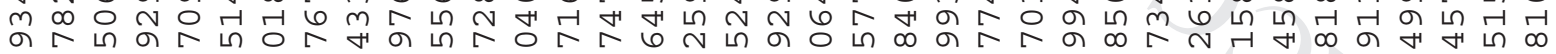

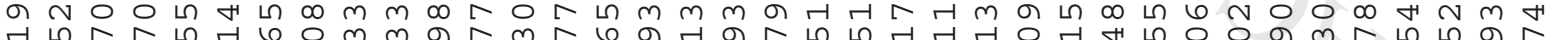

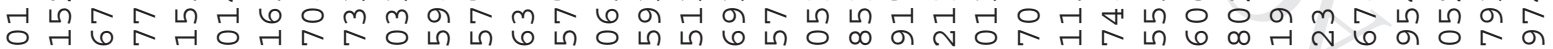

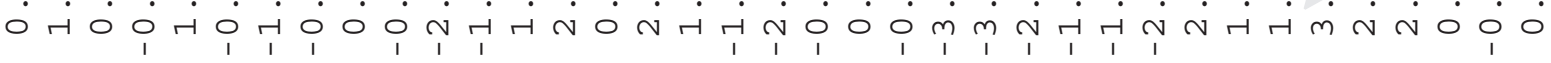

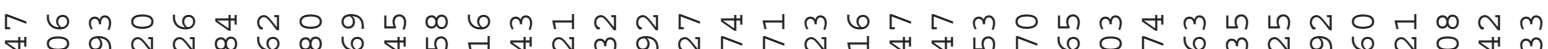

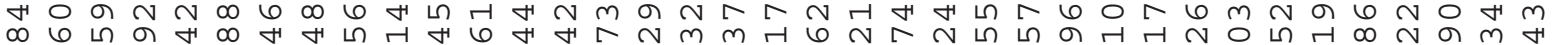

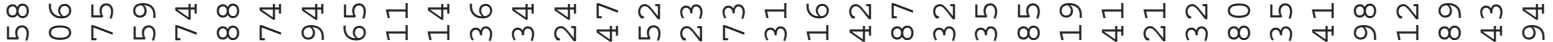

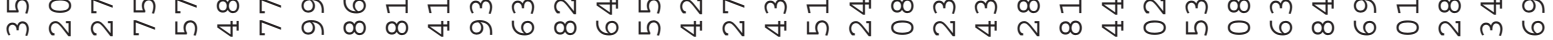

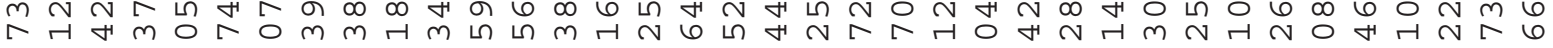

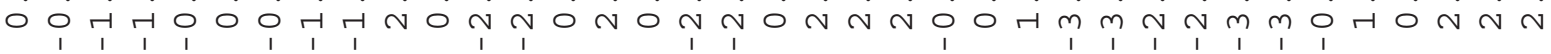

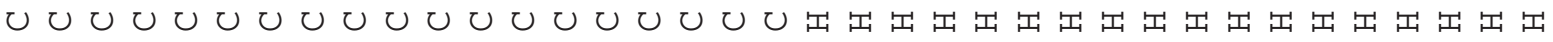
岑年 


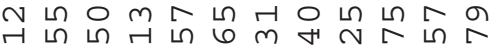

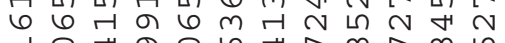
न अ 006 अ

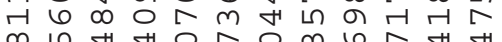
$\infty \pi$ L $\underset{1}{\sim} \dot{m}_{1} \underset{1}{\sim} \underset{1}{\sim} m_{1} \underset{1}{\sim} \underset{1}{\infty} \underset{1}{\sim} m_{1} \underset{1}{r}$

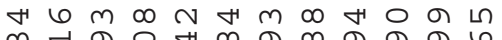

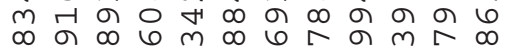
h $10 \mathrm{~m}$ H $\mathrm{r}$ \% H $6 \mathrm{~mm}$ त $\operatorname{rr}$ भ म

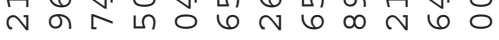
$m \sim \sim \sim r r_{1} \underset{1}{r_{1}} m_{1} \sim_{1}$

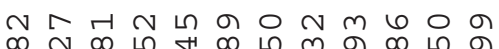

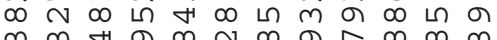

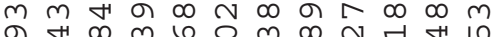

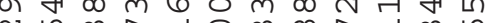

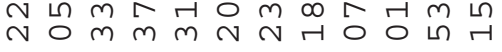

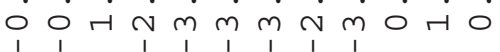

出出出出出出出出出出出出

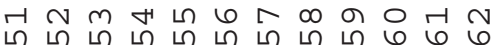

m の r

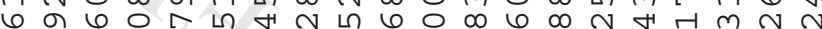

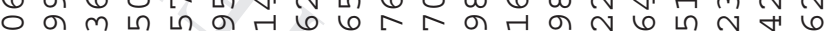
अ

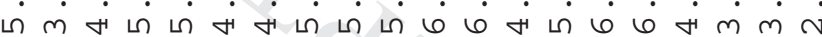

다

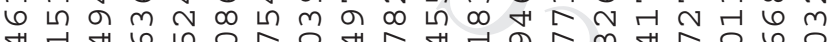
H $\infty$ म

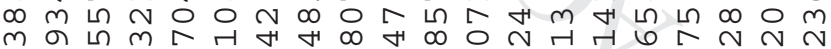

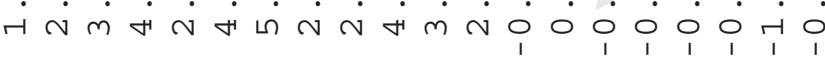

H4

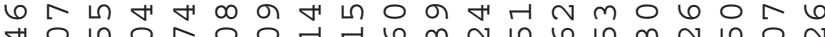

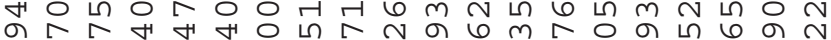

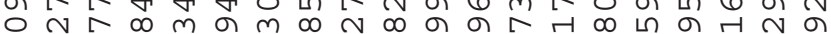

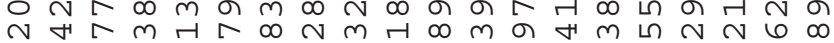

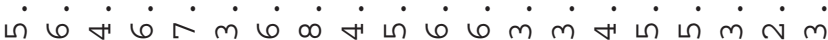

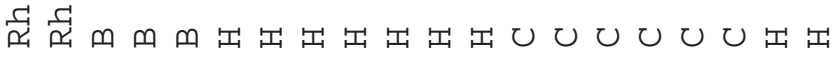

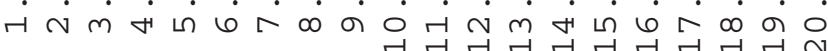




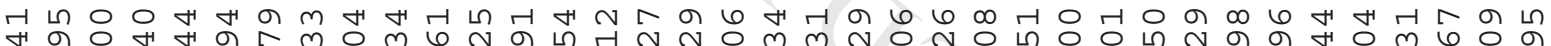

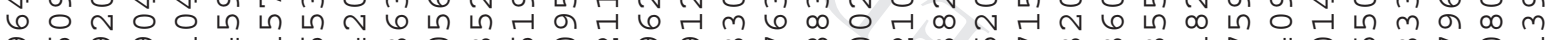
ब 6 ब न म

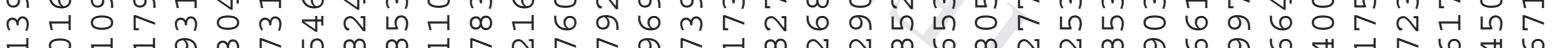

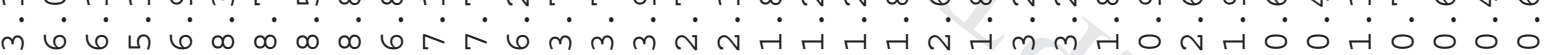

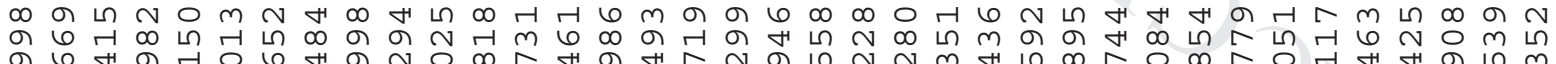
ब 6 H

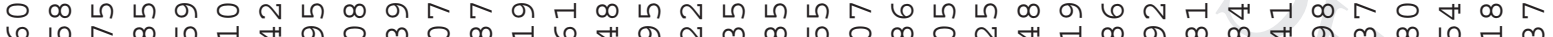
ம 0 0 0

$\infty$ H

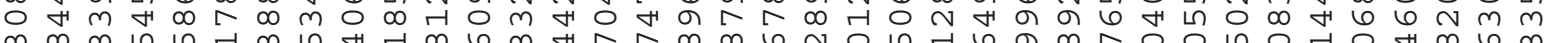

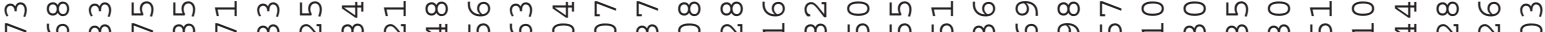
$\pi$ 다유

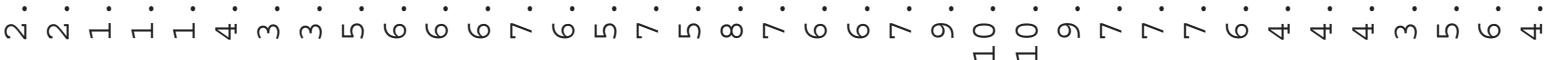

出U出出出U出出出U出出出U出出出UUUUUU出出出U出出出U出出出U出出

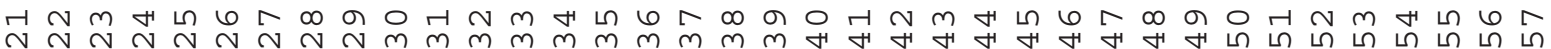


$\begin{array}{llll}4 & 6 & 6 & 6\end{array}$ a $m$ เ 6 6

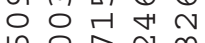
० ने के तं

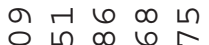
0 म $N$ in ด $\bigcirc \infty 6$ 윰 당 ๙ $\begin{array}{lllll}0 & 0 & 0 & 0 & 0\end{array}$

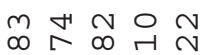
$m \sim m \in \approx$

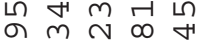

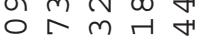
$6 \infty \sigma \infty \sigma$

出U出出出 ๓
앙다

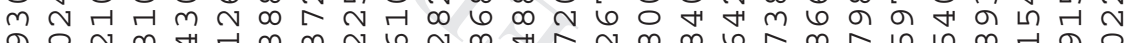

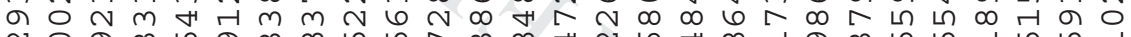

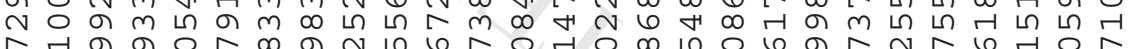

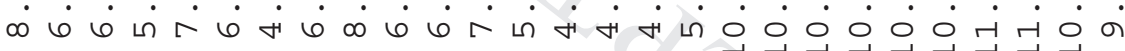

Иの ब $\sigma-10$

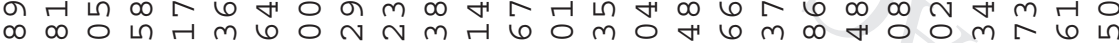

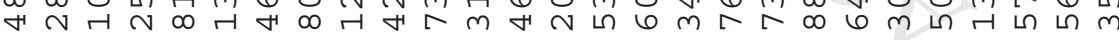

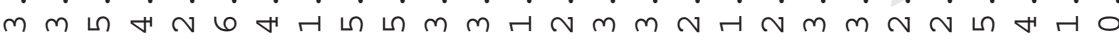

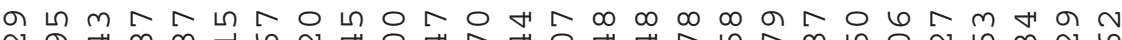
N H

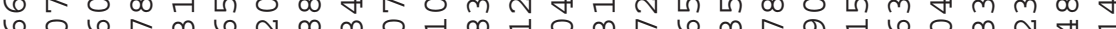

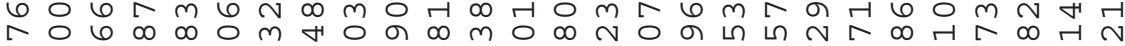

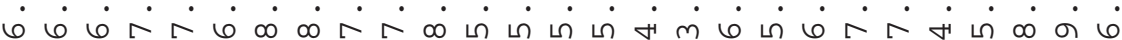

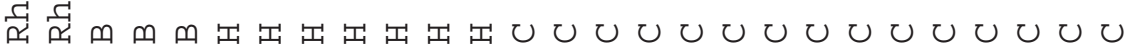

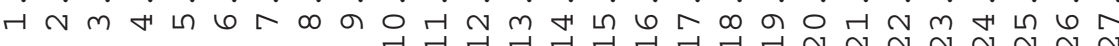




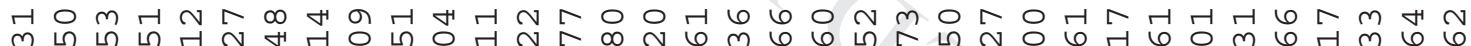

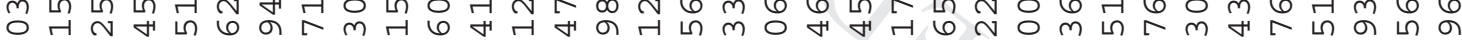

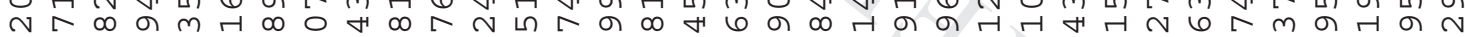

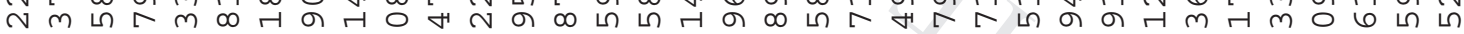
mo

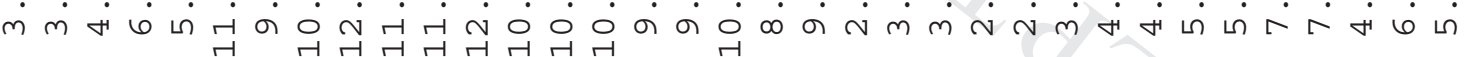

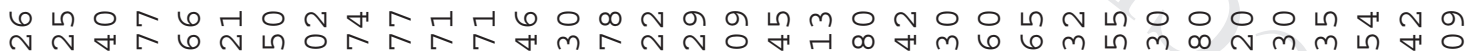

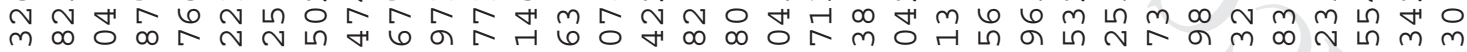

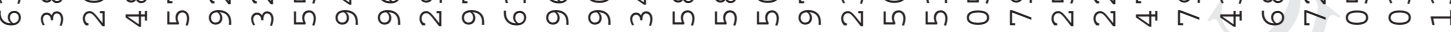
เ $\infty$ L เी

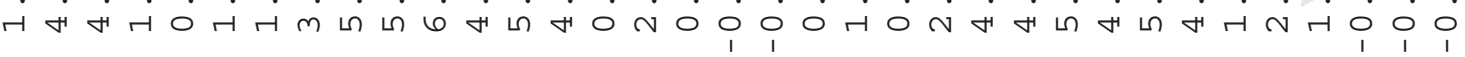

ஸ 윔 $\infty$ 근 $m$ क ने $m \leq 0 \leq 6)$

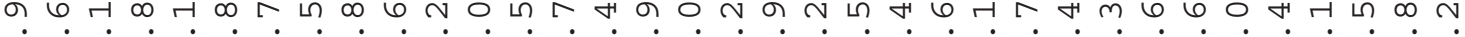
6 เ $m \sim$ เ $m m m$ เ

UUUU巳出出出出出出出出出出出出出出出出出出出出出出出出出出出出出出

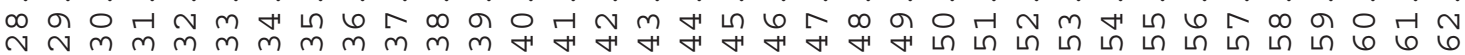




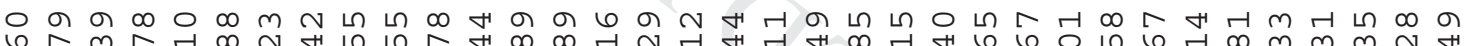

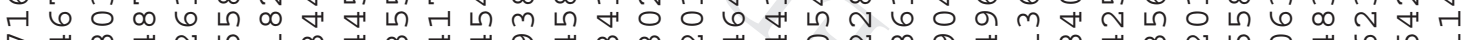
H $\begin{aligned} & n \\ & N\end{aligned}$

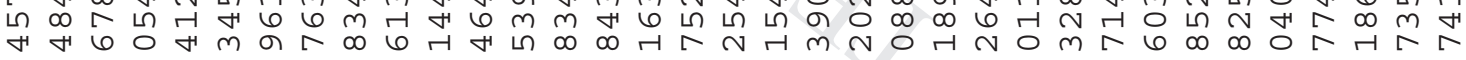

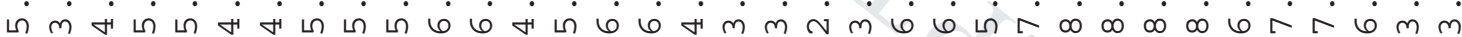

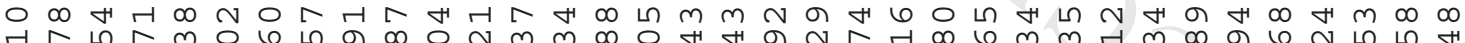
당 人 $m$ h

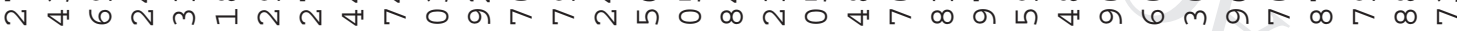
म

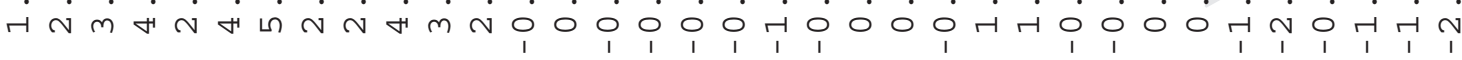

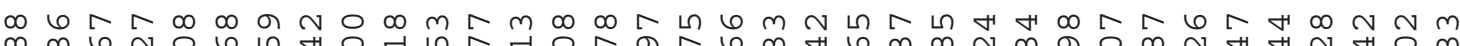

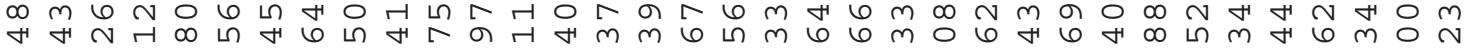

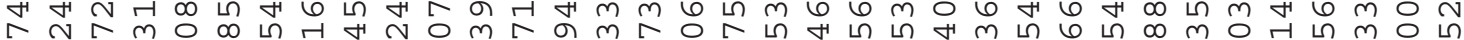

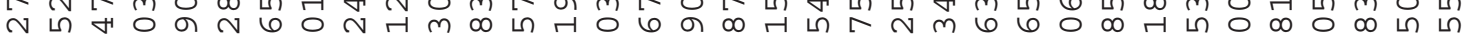
v

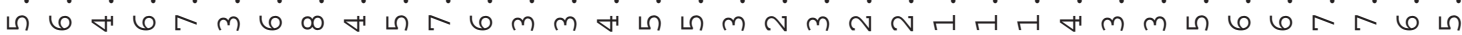

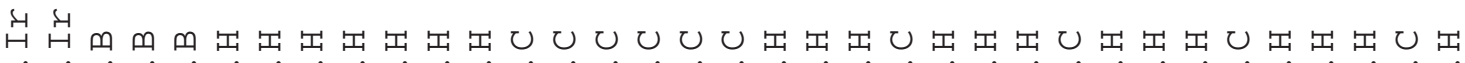

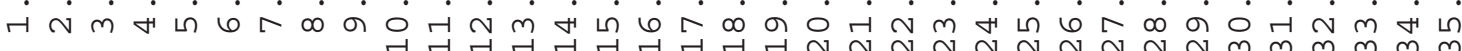


각 सम न ब 6 क ब 6 त

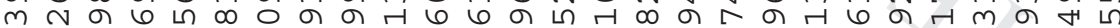
ar $\mathrm{N} N \mathrm{~N} \infty$ म

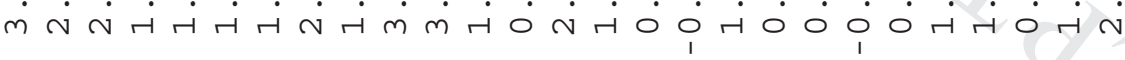

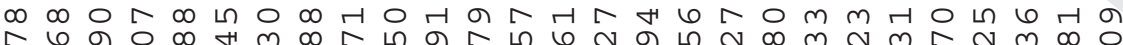

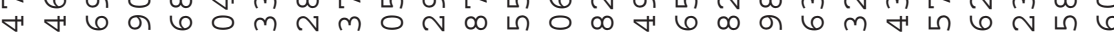

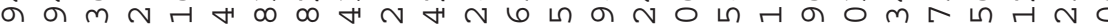
m नi

几

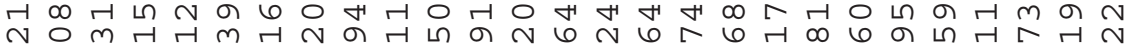

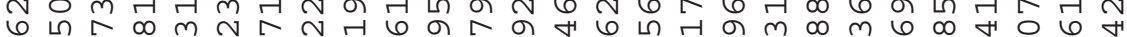

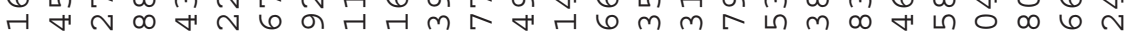
तु

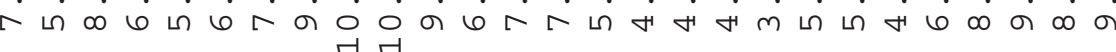

出出UUUUひU出出出U出出出U出出出U出出出U出出出

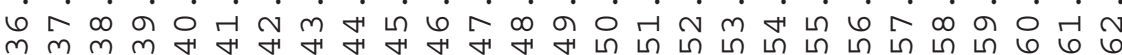

